\section{Cytogenetic and}

GenomeResearch

\title{
42nd Biennial American Cytogenetics Conference
}

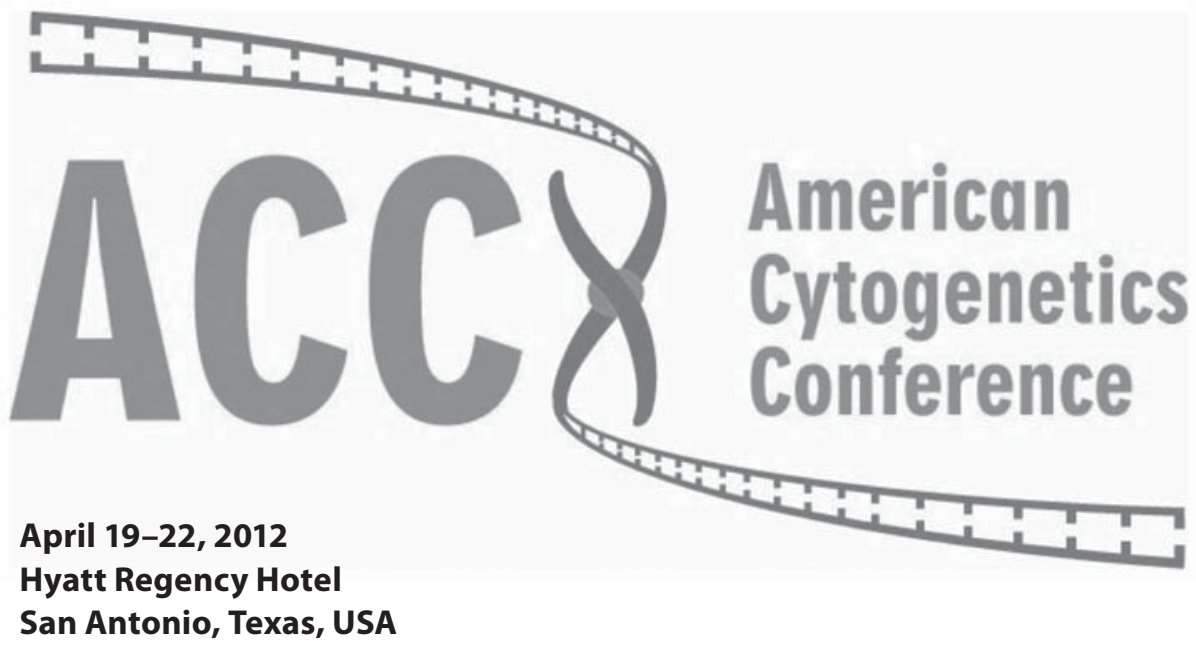

Meeting Chair:

Program Chair:
Charleen Moore

Patricia Howard-Peebles

American Cytogenetics Conference Distinguished Cytogeneticist Award:

Dr. Janet Davidson Rowley

Recipients of Student Travel Awards*:

A.V. Dharmadhikari - Baylor College of Medicine, Houston, TX

L.B. Henderson - Institute of Genetic Medicine, Johns Hopkins, Baltimore, MD

J.J. Roth - Department of Pediatrics, The Children's Hospital of Philadelphia, Philadelphia, PA

*Awards donated in honor of C.C. Lin, M. Ferguson-Smith, and C.C. Morton.

The 2010 American Cytogenetics Conference gratefully acknowledges the generous support of the following companies:

Abbott Molecular

Affymetrix, Inc.

Agilent Technologies

Applied Spectral Imaging, Inc.

BCM Medical Genetics Laboratories

BioDiscovery, Inc.

BlueGnome Ltd

Cartagenia

CymoGen Dx, LLC

Cytocell/Rainbow Scientific, Inc.
Genial Genetics/Rainbow Scientific, Inc.

Illumina, Inc.

InfoQuant

Irvine Scientific

ISCA Consortium

Kreatech, Inc.

Laboratory Corporation of America

Leica Microsystems Corp

MetaSystems Group, Inc.

Nikon Instruments, Inc.
Olympus America

Oxford Gene Technology

Phalanx Biotech Group, Inc.

Quest Diagnostics Nichols Institute

SCC Soft Computer

SciGene

Signature Genomics from PerkinElmer, Inc.

South Texas Reference Laboratories

Staff Icons

Transgenomic, Inc.

Refer to the back of the program for exhibitor contact information. 


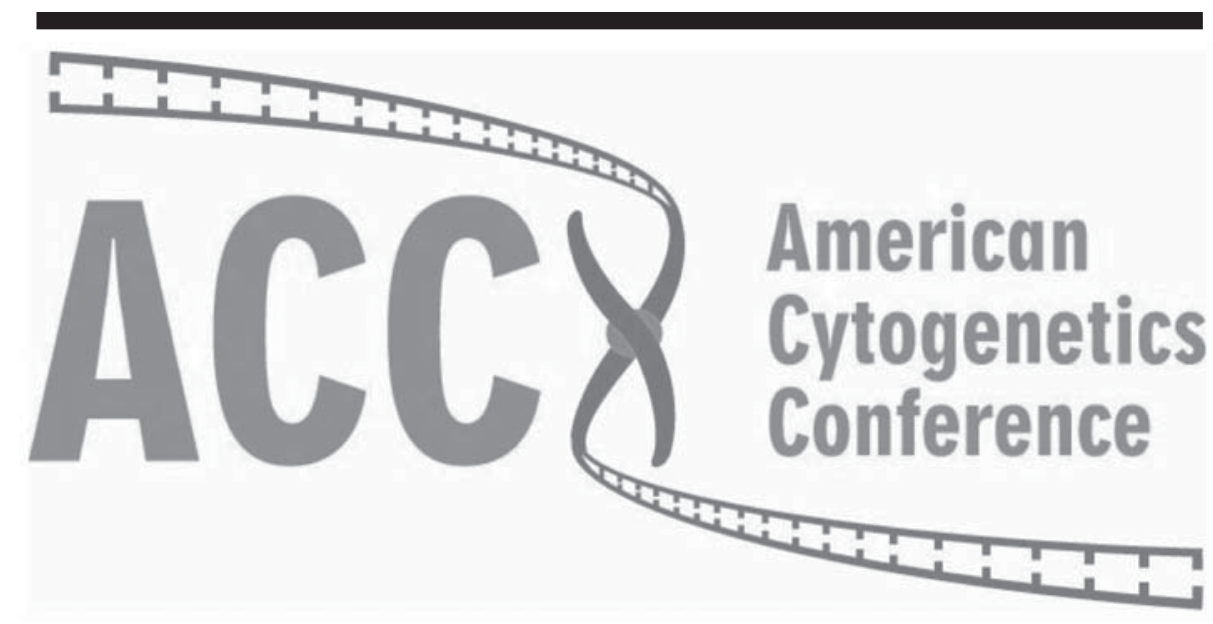

\section{Meeting Organizers and ACC Officers}

Program Committee

Distinguished Cytogeneticist

Committee

Local Arrangements

Committee

Nominating Committee
Patricia Howard-Peebles (Chair)

Robert Best

Jaclyn Biegel

Art Brothman

Catherine Rehder

Janice Smith

Daynna Wolff

Susan Zneimer

Diane Roulston (Co-Chair)

Janet Cowan (Co-Chair)

Art Brothman

Susan Olson

Denise Quigley

Charleen Moore (Chair)

Sue Ann Berend

Frank Grass

Peter Jacky

Debra Saxe

Charleen Moore (Chair)

Sue Ann Berend

Betty Dunn

Patricia Howard-Peebles

Gopalrao Velagaleti
Student Awards

Corporate

Sponsorships

Membership

Publicity

Registration

Website

Historian

Continuing Education

\section{Board of Directors:}

Denise Quigley

Sarah South

Jim Tepperberg
Sarah South (Chair)

Caroline Astbury

Urvashi Surti

Barbara DuPont (Chair)

Gail Wenger

Colleen Jackson-Cook (Chair)

Frank Grass (Co-Chair)

Wendy Golden (Co-Chair)

Sue Ann Berend (Chair)

Karla Gay

Joey Kelly

Denise Quigley (Chair)

Charleen Moore

AnneMarie Block (Chair)

Athena Cherry

Sheila Dobin
President: Charleen Moore

Vice President: Hutton Kearney

Secretary/Treasurer: Sue Ann Berend

Past President: Avirachan Tharapel
Preprint Cytogenet Genome Res 136/4/2012

Abstracts will be available online, free of charge 


\title{
2012 Recipient ACC Distinguished Cytogeneticist Janet Davidson Rowley, MD
}

\author{
Blum-Riese Distinguished Service Professor of Medicine, \\ Molecular Genetics and Cell Biology, and Human Genetics, \\ University of Chicago, IL
}

During this 2012 American Cytogenetics Conference, we honor the career of Janet Davidson Rowley, a physician, scientist, and a founder of the field of modern cancer cytogenetics. Her contributions span over half a century and are represented by more than 400 publications, numerous awards and honors, among which are the American Society of Human Genetics Allan Award and the Presidential Medal of Freedom. Dr. Rowley has been a driving force for understanding the biological importance of chromosomal translocations and much of her most significant work has influenced the way leukemias and lymphomas are diagnosed and treated. Her seminal discoveries of translocations associated with different types of leukemia have become part of the standard of care in determining the optimal treatment for every leukemia patient. Her work on translocation breakpoints has shaped our biological understanding of the role of genes in cancer and has provided tools to track the effectiveness of treatment. Dr. Rowley's study of translocations was also instrumental in the expansion of the fields of molecular genetics and molecular oncology and her continuous quest for the answers to fundamental questions led directly to the work she does now in the molecular biology of microRNAs.

Part of Janet Rowley's success entails a woman who was at the right place at the right time. In 1940, she received a scholarship to college in a special program at the University of Chicago which combined the last two years of high school with the first two years of college. Thus, she enrolled as an undergraduate at age 15 and earned a

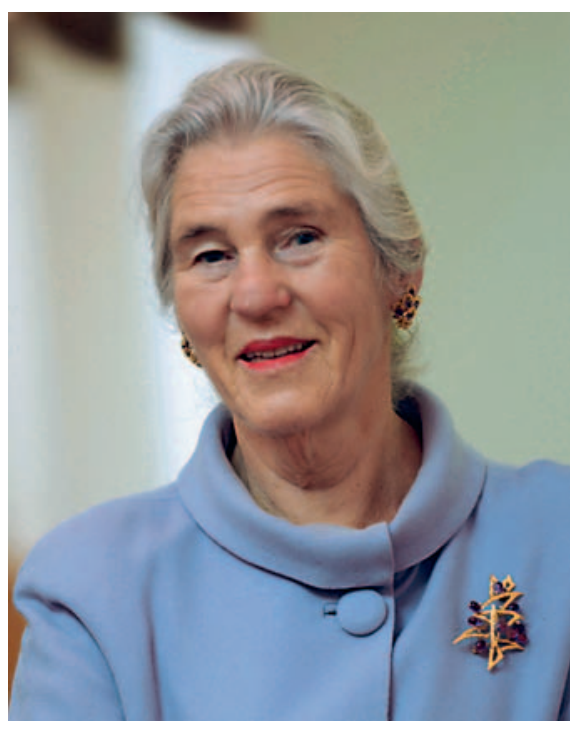

PhB (1944), BS (1946) and MD (1948) at the University of Chicago. Her education in medicine did not start so easily; her entrance to medical school was delayed by a year because the quota for women (three) was filled. She graduated in 1948 and a day after graduation she married Donald Rowley. Janet completed a one-year internship at a Public Health Service hospital before moving to Bethesda, Maryland, where Don had accepted a position at the NIH. After three years, the couple returned to Chicago and Janet resumed her work part-time in well-baby clinics, when Don began working as an experimental immunologist on faculty at the University of Chicago. In 1956, 
Janet took a job at a Cook County Hospital clinic working with children with developmental disabilities such as Down syndrome. Soon after, trisomy 21 was discovered to cause Down syndrome and she was inspired to study cytogenetics as a medical specialty.

Janet got her first chance to conduct research when Don took a sabbatical year in Oxford in 1961. Ever resourceful, she won a special fellowship from the NIH to study cytogenetics at Churchill Hospital in Oxford. This was extremely fortuitous timing, as the field was in its infancy and scientists were just beginning to explore chromosome replication. Her productivity was amazing - between 1962 and 1964, she published four seminal papers, and was first author on two (cited in Rowley, 2009). But what is more amazing is how she managed to do the analysis at this early stage in her career while also caring for four children.

After returning to Chicago, she obtained support from Leon Jacobson, a hematologist who had become an expert on the effect of radiation on blood cells from monitoring physicists working on the Manhattan project, just a couple of blocks from the University medical center. Thanks to a stipend from the Atomic Energy Commission, she could analyze the chromosomes prepared in Sweden and England. Janet rose through the ranks becoming Professor of Medicine at the University of Chicago in 1977. She is currently the Blum-Riese Distinguished Service Professor of Medicine, Molecular Genetics and Cell Biology, and Human Genetics.

It was after the family's second stay in Oxford during 1970-1971 that Janet made her most significant discoveries. At Oxford she learned banding by quinacrine fluorescence and applied this technique upon returning to Chicago. Within the next couple of years she demonstrated that the small Philadelphia chromosome was actually a translocation between two chromosomes, hence characterizing for the first time the $t(9 ; 22)$. She had already described the recurring abnormality $t(8 ; 21)$ in 1972 , and this second translocation convinced her that these were critically important in leukemia. Discovery of the $t(15 ; 17), t(6 ; 9)$ and $t(14 ; 18)$ in lymphoma would soon follow.
Janet states in a detailed review (Rowley, 2009), 'This was a time of great excitement and wonder that is hard to describe.' Collaborating with many colleagues, including those involved with the International Workshops during the 1970's and 1980's, several main themes emerged which changed the way researchers and clinicians treat leukemia and lymphoma. Not only are there recurrent chromosome abnormalities, but they often strongly associate with specific phenotypes of leukemia and lymphoma, including the presence of cytogenetic-clinicopathologic entities. The phenotype even extends to how well patients respond to therapy, and the karyotype is a powerful independent predictor of survival.

In the three decades since, Dr. Rowley and her colleagues have discovered the MLL gene, characterized genomic breakpoints, and used cutting-edge microarray technology in the study of leukemia translocations. More recently, she has entered into the world of microRNAs. Her pace has not slowed in the lab; her goal remains to find the best treatments for leukemia: targeted and personalized. She has continued to pursue the answers to fundamental questions yet to be resolved: what causes translocations, and what are the critical steps of leukemogenesis?

As noted above, Janet Rowley has received many awards. We are very pleased to now add the American Cytogenetic Conference's Distinguished Cytogeneticist Award to this list.

\section{Further Reading: Reminiscences and Summaries of} Early Work

Chandra HS, Heisterkamp NC, Hungerford A, Morrissette JJD, Nowell PC, Rowley JD, Testa JR: Philadelphia chromosome symposium: commemoration of the 50th anniversary of the discovery of the Ph chromosome. Cancer Genet 204:171-179 (2011).

Rowley JD: Chromosomal translocations: revisited yet again. Blood 112:2183-2189 (2008).

Rowley JD: Chromosomes in leukemia and beyond: from irrelevant to central players. Annu Rev Genomics Hum Genet 10:1-18 (2009). 


\title{
Scientific Program
}

\author{
Thursday, April 19 \\ 11:30 am-1:30 pm ACC Board Meeting, Pecan Room \\ 2:30-5:00 pm \\ Exhibitor Registration and set-up, Rio Grande Ballroom \\ 3:00-6:00 pm \\ Conference Registration, Los Rios Foyer \\ Poster set-up, Rio Grande Ballroom \\ 5:30-7:00 pm \\ Opening Reception, Rio Grande Ballroom \\ Sponsored by Leica Microsystems Corp
}

\section{Friday, April 20}

7:00 am-noon

7:00 am-noon

Conference Registration, Los Rios Foyer

7:00 am-5:30 pm

Poster set-up, Rio Grande Ballroom

Exhibits open, Rio Grande Ballroom

Please visit the booths, especially during the opening reception,

continental breakfasts, breaks, lunchtime and poster sessions.

$\begin{array}{ll}\text { 7:00-9:00 am } & \begin{array}{l}\text { Continental Breakfast, Rio Grande Ballroom } \\ \text { Sponsored by Cytocell/Rainbow Scientific, Inc. }\end{array} \\ \text { 2:45-4:15 pm } & \text { Poster Session, Rio Grande Ballroom }\end{array}$

All Scientific Sessions will take place in the Regency East Ballroom

8:00-8:15 am Welcome Address: Charleen Moore - President

and Patricia Howard-Peebles - Program Chair

\section{Scientific Session 1: Constitutional Cytogenetics I}

Moderators: Daniel Van Dyke and Jennifer Laffin

8:15-8:30 am

1 Chromosomal Breakpoints Target a Variety of Functional Elements in the Human Genome, Including Annotated, Unannotated, Coding and Non-Coding Transcriptional Regions

N. Tommerup, I. Bache, N.M. Nielsen, S. Kjaergaard, K. Brøndum-Nielsen, M. Frisch, A. Lind-Thomsen, Y. Mang, C. Hansen, A. Silahtaroglu, M. Kirchhoff, P.K.A. Jensen, C. Fagerberg, L.N. Krogh, J. Hansen, T. Bryndorf, Z. El-Schich, K.F. Henriksen, C.M. Anderson, M. Bak, C. Halgren

8:30-8:45 am

2 The Impact of Chromosomal Microarray on Clinical Management

L.B. Henderson*, E. Wohler, C.D. Applegate, D.A.S. Batista

*Student presenter - Award winner

8:45-9:00 am

3 Microarray Analysis of X Chromosome Aberrations in 45 Women with Highly Skewed X Inactivation and 45 Controls

9:00-10:00 am

V. Jobanputra, B. Levy, A. Kinney, S. Brown, M. Shirazi, C.-Y. Yu, J. Kline, D. Warburton

9.00-10.00 am

4 Weird Animal Genomes, Sex and the Future of Men

Invited Speaker: Jennifer Marshall Graves

Professor Emeritus, The Australian National University, Canberra

Introduced by Art Brothman

Sponsored by Signature Genomics from PerkinElmer, Inc.

10:00-10:30 am

Coffee Break - Rio Grande Ballroom

10:00-10:30 am

Sponsored by Laboratory Corporation of America

View Posters and Exhibits - Rio Grande Ballroom

Preprint Cytogenet Genome Res 136/4/2012 
Scientific Session 2: Constitutional Cytogenetics II

Moderators: Charles Lee and Jennifer Winters

10:30-10:45 am 5 Utility of SNP Arrays in Detecting, Quantifying, and Determining Meiotic Origin of Tetrasomy 12p in Blood from Pallister-Killian Patients

L.K. Conlin, K. Izumi, M. Kaur, D. Clark, A. Wilkens, E. Zackai, M.A. Deardorff, N.B. Spinner, I.D. Krantz

10:45-11:00 am

6 Small Recurrent Deletions and Reciprocal Duplications in 2q21.1, Including Brain-Specific ARHGEF4

and GPR148, in Patients with Developmental Delay, ADHD, Autism, and Epilepsy

A.V. Dharmadhikari*, S.-H.L. Kang, P. Szafranski, R.E. Person, S. Sampath, S.K. Prakash, P.I. Bader, J.A. Phillips III, V. Hannig,

M. Williams, S.S. Vinson, A.A. Wilfong, W.J. Craigen, J. Wiszniewska, A. Patel, W. Bi, J.R. Lupski, J. Belmont, S.W. Cheung,

P. Stankiewicz

*Student presenter - Award winner

11:00-11:15 am

7 Next Generation Approaches to the Analyses of Inversions

S.M. Bailey, E. Zimmerman, M.N. Cornforth, J.S. Bedford, E.H. Goodwin, F.A. Ray

11:15-11:30 am $\quad 8$ De novo Copy Number Losses of Chromosomes 2q22.3q23.2 and 5q11.2q12.2 in a Female Patient with Clinical Features of Angelman Syndrome: A Case Report

A.D. Chaubey, F. Bartel, S.A. Skinner, B.R. DuPont

11:30-11:45 am 9 Maternal Uniparental Disomy of Chromosome 7 in a Patient with Myoclonus-Dystonia

M.B. Sheridan*, A.B. Telegrafi, V. Stinnett, T.M. Dawson, J. Bodurtha, D.A.S. Batista

*Student presenter

$11: 45-12: 00 \mathrm{pm}$

10 A Four Generation Family with Duplication of 17p13.3 with Variable Split-Hand/Foot Malformation

12:00-1:30 pm

B.R. DuPont, A.D. Chaubey, F.O. Bartel, K.R. Holden, R. Alvarenga, D.K. Simpson, C.M. Armour, C.E. Schwartz, D.B. Everman Lunch on your own - Exhibitor area is open to all attendees during lunch

\section{Scientific Session 3: Non-Human Cytogenetics}

Moderators: Katie Rudd and Marlys Houck

1:30-1:45 pm 11 Development and Application of Molecular Tools for Camelid Cytogenetics

F. Avila*, P.J. Das, M. Kutzler, E. Owens, P. Perelman, T. Raudsepp

*Student presenter

1:45-2:00 pm 12 Noncoding RNAs and Chromosome Change [Withdrawn: Replaced by Abstract 68]

R.J. O'Neill

2:00-2:15 pm

13 Identification and Expression Analysis of the Pig Pseudoautosomal Region

Using Molecular Cytogenetics Approach

P.J. Das*, D.K. Mishra, F. Avila, G. Johnson, B.P. Chowdhary, T. Raudsepp

*Student presenter

2:15-2:30 pm

14 Devil Facial Tumour Disease: Does Chromosome 1 Hold the Key?

J.E. Deakin, Y. Zhang

2:30-2:45 pm

15 Array CGH Studies in Equine Disorders of Sexual Development - SRY-Positive Sex Reversal and Cryptorchidism S. Ghosh*, P.J. Das, Z. Qu, E. Fang, C. Arnold, D.L. Adelson, B.P. Chowdhary, T. Raudsepp

*Student presenter

2:45-4:15 pm Coffee Break - Rio Grande Ballroom

Sponsored by Illumina, Inc.

2:45-4:15 pm

Poster Session and Exhibits - Rio Grande Ballroom

2:45-3:30 pm

Authors present at odd numbered posters

Authors present at even numbered posters

\section{Scientific Session 4: Cancer Cytogenetics I}

Moderators: Daynna Wolff and Lynne Abruzzo

4:15-4:30 pm 16 Dicentric Chromosomes Are Frequent in Chronic Lymphocytic Leukemia (CLL)

N.A. Heerema, H. Breidenbach, C. Miller, A. McFaddin, S. Haudenshield, N. Muthusamy, J.C. Byrd

4:30-4:45 pm

17 Diagnostic Application of High Resolution SNP Arrays for Children with Brain Tumors

J.J. Roth*, M.J. Dougherty, L.S. Tooke, A.F. Teplick, J.A. Biegel

*Student presenter - Award winner

4:45-5:00 pm

18 Metaphase Induction in Multiple Myeloma: A New Cytogenetic Approach for G-Banding Analysis

F. Morato de Oliveira, A.P.N.R. Alves, E.M. Rego, R.P. Falcão

5:00-5:15 pm 19 Separation of Myeloid and T-Cells prior to FISH Analysis in Blood Samples from Patients Undergoing Hematopoietic Stem Cell Transplantation

H. Aviv, S. Payne-Blackman, I. Maxwell, M. Osman

Preprint Cytogenet Genome Res 136/4/2012

Abstracts will be available online, free of charge 
5:15-5:30 pm 20 A Comparison of FISH and Microarray Detection of Mosaicism in ALL, CLL, and MDS

M.C. Sederberg, G. Gu, C.N. Paxton, L.R. Rowe, K.B. Geiersbach, S.T. South

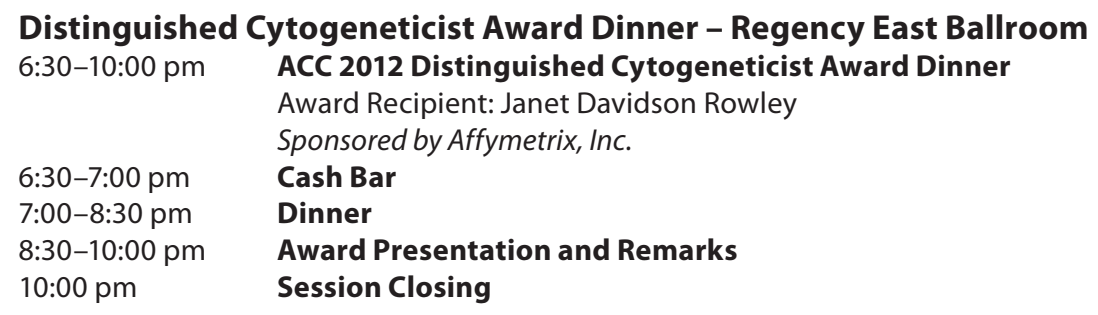

Saturday, April 21

7:00-9:00 am Continental Breakfast - Rio Grande Ballroom

Sponsored by Kreatech, Inc.

7:00-noon Conference Registration - Los Rios Foyer

Scientific Session 5: Constitutional Cytogenetics III

Moderators: Hutton Kearney and Colleen Jackson-Cook

8:00-8:15 am 21 The Carolinas Array Group Database (CAGdb): A Platform-Independent Database of Microarray Findings Shared across Multiple Institutions to Aid in Laboratory Data Management, CNV Interpretation and Submission to ISCA/NCBI ClinVar Datasets

H.M. Kearney, W.P. Allen, A. Chaubey, B.R. DuPont, K.A. Kaiser-Rogers, C.W. Rehder, S.T. South, J. Tepperberg, D.J. Wolff, J. Paschall, S. Lassiter, T.J. Hill

8:15-8:30 am 22 Insights from Prenatal Array CGH Analysis: Impact of Cell Culture on Results

J.M. Meck, L. Matyakhina, L. Schmidt, V. Nelson, S. Warren, K. Redford, G. Richard, S. Aradhya

8:30-8:45 am 23 Towards a Universal Database of Human Genomic Variation: Lessons Learned from the ISCA Consortium

E.R. Riggs, E.B. Kaminsky, V. Patel, W.A. Faucett, E. Thorland, D. Church, J. Paschall, L. Jackson, S. Aradhya, R. Kuhn,

R. Nussbaum, H. Rehm, R. Wapner, D.H. Ledbetter, C.L. Martin

8:45-9:00 am 24 Somatic Mosaicism Detected by Exon-Targeted, High-Resolution aCGH in 10,362 Consecutive Cases J. Pham, C. Shaw, P. Hixson, A. Ester, A. Pursley, S.S.-H. Kang, W. Bi, S. Lanani, C. Bacino, P. Stankiewicz, A. Patel, S.W. Cheung

9:00-10:00 am 25 Embryo and Fetal Diagnostics: The Power of SNP Microarrays

Invited Speaker: Brynn Levy

Associate Professor, Center for Human Genetics, Columbia University Medical Center, New York, N.Y.

Introduced by Jaclyn Biegel

10:00-11:00 am Coffee Break - Rio Grande Ballroom

Sponsored by Applied Spectral Imaging, Inc.

10:00-11:00 am View Posters and Exhibits - Rio Grande Ballroom

Last chance to view posters and talk with exhibitors

\section{Scientific Session 6: Cancer Cytogenetics II}

Moderators: Sheila Dobin and Nyla Heerema

11:00-11:15 am

26 Detection of Masked Hypodiploidy in Pediatric B-Cell Precursor Acute Lymphoblastic Leukemia by Single Nucleotide Polymorphism Arrays

X.-Y. Lu, A. Patel, J. Ringrose, Y.-J. Zhao, P. Rao, S.E. Plon, K.R. Rabin

11:15-11:30 am

27 An Increased Frequency of Micronuclei in Lymphocytes Is Associated with a Diagnosis of Breast Cancer and the Acquisition/Persistence of a Subset of Chemotherapy-Related Psychoneurologic Symptoms

N. Aboalela*, D. Lyon, R. Elswick, Y.-J. Chen, C. Jackson-Cook

*Student presenter

$11: 30-11: 45$ am

28 Genomic Variability by Whole-Genome SNP Genotyping Predicts Time-to-Event Outcome in Previously Untreated Chronic Lymphocytic Leukemia (CLL) Patients

C.D. Schweighofer, K.R. Coombes, T. Majewski, L.L. Barron, S. Lerner, W.G. Wierda, A. Ferrajoli, S. O'Brien, L.J. Medeiros,

B.A. Czerniak, M.J. Keating, L.V. Abruzzo

11:45-12:00 pm 29 Combination of Fluorescence in situ Hybridization (FISH) and Cytogenetic Techniques Optimizes the Diagnostic Process of Patients with Myelodysplastic Syndrome (MDS)

L. Trakhtenbrot, D. Merkel, I. Novikov, A. Nagler, N. Amariglio

12:00-12:15 pm 30 Conventional Cytogenetic Analysis and FISH in Myelodysplastic Syndrome (MDS): Are Both Necessary? A.E. Wiktor, D.L. Van Dyke, R.P. Ketterling, C.A. Hanson 


\section{Afternoon on your own}

$12: 30 \mathrm{pm}$

6:30-11:00 pm

Departure for optional activities (box lunch pickup) - Los Rios Foyer

Fiesta Buffet and Entertainment - Regency East Ballroom

Sponsored by Quest Diagnostics Nichols Institute

Sunday, April 22

7:00-9:00 am

Continental Breakfast - Regency East Ballroom Foyer

Sponsored by Abbott Molecular

8:15-8:45 am ACC Business Meeting - Regency East Ballroom

With student award presentations

Scientific Session 7: Cancer Cytogenetics III

Moderators: Sarah South and Jaclyn Biegel

8:45-9:00 am

31 Transient Clonal Cytogenetic Abnormalities following Chemotherapy for Acute Leukemia

F. Racke, N. Heerema

9:00-9:15 am

Constitutional t $(16 ; 21)(p 13 ; q 22)$ Identified by FISH and Next-Generation Genome-Wide Long Mate-Pair Sequencing Analysis as a Novel Pathogenomic Mechanism in a Series of Three New RUNX1 Mutated FPD/AML Pedigrees: Implications for Diagnostics

A. Buijs, M. Poot, S. van der Crabben, B. van der Zwaag, E. van Binsbergen, M.J. van Roosmalen, M. Tavakoli-Yaraki, O. de Weerdt, H.K. Nieuwenhuis, M. van Gijn, W.P. Kloosterman

9:15-9:30 am 33 Comparison of Two Microarray (aCGH) Platforms to Assess Global Genomic Status in B-Chronic Lymphocytic Leukemia (CLL)

J.G. Rustin, D.L. Van Dyke, S.A. Smoley, E.C. Thorland, E. Braggio, N.E. Kay

9:30-9:45 am

34 Genomic Profiling of Rare Disseminated Tumor Cells from Prostate Cancer

M. Fang, J. Schoenborn, J. Xia, C. Morrissey, P. Nelson, R. Vessella

9:45-10:00 am $\quad 35$ Genomic Microarray Analysis Provides an Excellent Genetic Assessment for Oncology: Preliminary Results of the Cancer Cytogenomics Microarray Consortium's Cross-Platform Study

D.J. Wolff, J. Biegel, J. Hagenkord, V. Jobanputra, J. Laffin, B. Levy, M.M. Li, P.M. Miron, F. Monzon, G. Toruner, M. Rossi, I. Znoyko, L. Tooke, D. Wilmoth, M. Dougherty, S. Kash, V. Aggarwal, O. Nahum, P. Chen, X. Hu, M. Cipollini, K. Alvarez, D. Streck

10:00-11:00 am 36 Insights into the Biology of Acute Leukemia from Genomic Profiling and Next-Generation Sequencing Invited Speaker: Charles G. Mullighan

Associate Member, Department of Pathology, St. Jude Children's Research Hospital, Memphis, Tenn. Introduced by Robert Best

11:00-11:30 am

Coffee Break - Regency East Ballroom Foyer

Scientific Session 8: Constitutional Cytogenetics IV

Moderators: Denise Batista and Janice Smith

11:30-11:45 am

37 Cytogenetic Biodosimetry: A Nuclear Countermeasures Strategy for the U.S.

G.K. Livingston, D.L. Van Dyke

11:45-12:00 am 38 Alterations in the CDKL5 and MECP2 Genes in Patients with Atypical Phenotypes

H. Mason-Suares*, C.L. Martin, M.K. Rudd

*Student presenter

12:00-12:15 pm 39 Adult Identical Twins Discordant for a History of Childhood Sexual Abuse Show Higher Frequencies of Acquired Chromosomal Changes in the Abused Twins

J. Brumelle*, T.P. York, J. Juusola, C. Jackson-Cook

*Student presenter

12:15-12:30 pm $\quad 40$ Microarray Analysis Reveals Mosaicism for Two Abnormal Cell Lines in a Patient with Previously Diagnosed Chromosome 9p Deletion

D.I. Quigley, M.I. Dewitt, V.J. Payne, O.A. Lamb, C. Miranda, D.K. Simpson

12:30-12:45 pm 41 High Density SNP Microarray Analysis of Products of Conception

K.B. Geiersbach, C.N. Paxton, A.N. Lamb, A.R. Brothman, S.T. South

$12: 45-1: 00 \mathrm{pm}$

42 SNP POC Microarray Analysis - Primary Cytogenetic Test for POC

J. Tepperberg, H. Taylor, R.D. Burnside, B. Rush, I.K. Gadi, V. Jaswaney, E. Keitges, R. Pasion, K.L. Phillips, V.R. Potluri, H. Risheg, S. Schwartz, J.L. Smith, P.R. Papenhausen

1:00-1:15 pm

Preprint Cytogenet Genome Res 136/4/2012

Abstracts will be available online, free of charge 


\section{Poster Presentations}

Poster set up times are Thursday 3:00-6:00 pm

Friday 7:00 am-Noon

Designated times for authors to be at their posters are:

Friday 2:45-3:30 pm - Odd numbered posters

Friday 3:30-4:15 pm - Even numbered posters

\section{Constitutional Cytogenetics}

43 Microdeletion of 17q24.3 Detected by Chromosome Microarray Analysis Is Associated with Pierre Robin Sequence due to Position Effect on SOX9

I.E. Amarillo, K. Dipple, F. Quintero-Rivera

44 Molecular and Clinical Characterization of Patients with Tetrasomy and Pentasomy 15q11q13

L. Bao, J.Y. Yang, Y. Yang, Y. Huang, H. Li

45 The Next Step in Clinical Cytogenetics: Moving Beyond Empirical Rules R.G. Best, G. Khushf

46 A Comparison of FISH and Microarray Technologies for Detecting Mosaicism for Trisomy 21

C. Charalsawadi*, P. Papavasiliou, C. Dumur, A. Ferreira-Gonzalez, T.P. York, C. Jackson-Cook *Student presenter

47 Abnormal Recombination between Yp11.2 and Xq21.3 Homology Loci as a New Mechanism of X-Y Translocations and Terminal Xq21.3/Xq22 Deletions

V.B. Chernykh*, L.F. Kurilo, V.A. Galkina, M.E. Karmanov, Y.O. Kozlova, M.E. Minzhenkova, O.P. Ryzhkova, A.L. Chukhrova, A.V. Polyakov *Student presenter

48 Double Autosomal Trisomy in a Liveborn Term Fetus: 47, XY,+9/47,XY,+18

P.T. Greipp, E.B. Mitchell, M.J. Wick

49 Comprehensive Assessment and Validation of the Automated Metaphase Finder GSL-120 J.C. Hodge, C.L. James, D.A. Duncan, R.C. Mulder

50 Mosaicism of a Normal Female Karyotype and an Unbalanced Autosomal Reciprocal Translocation K. Huijsdens-van Amsterdam, M. van Seggelen, M.C. van Maarle, C.J. Bax, A.C. Knegt

51 Autosomal Structural Mosaicism - A Review of Cases from Eleven Years of Constitutional Karyotyping Experience A.M. Joseph-George, D. Antinucci, G. Nie, I. Teshima, D.J. Stavropoulous, M. Shago

52 The Triumphs and Challenges of Very High Density SNP Microarray Analysis in a Non-Academic Clinical Setting J. Kogan, C. Booth, D. Rita, A. Catic

53 Phylloid Hypomelanosis in a Patient with Mosaic Tetrasomy 13q A.S. Kulharya, V.S. Tonk, J.N. Myers Jr

54 Interstitial 12q24.31 Deletion Detected by Array CGH in a Girl with Neonatal Hyperinsulinism, Childhood Diabetes Mellitus Type 2, Dysmorphism, Developmental Delay, and Behavioral Problems F. Li, K.J. Holbrook, X. Li, D.R. Witt

55 Prenatal Detection and Characterization of a psu idic(8)(p23.3) Which Likely Derived from a Nonallelic Homologous Recombination between Two MYOM2 Repeats

Y.-C. Li, S.-C. Chien, S.R. Setlur, W.-D. Lin, F.-J. Tsai, C.-C. Lin

56 Copy Number Changes and Phenotypes Associated with Seizures in a Cohort Analyzed by High Density Whole Genome Array L. Matyakhina, J.M. Meck, S. Warren, S. Aradhya

57 Epigenetic Exception of X Inactivation Reversal during Gametogenesis F. Nouraei, N. Rao, B. Rashidi, I. Filip, A. Radfar

58 Meiotic Segregation Pattern and Chromatin Structure Analysis of Spermatozoa from a Carrier with Complex Chromosome Rearrangement (CCR) t(6;10;11)(q25;q24;q23) - Case Report

M. Olszewska*, N. Huleyuk, D. Zastavna, E. Wiland, M. Kurpisz

*Student presenter

59 Identification of a Novel Recurrent Microdeletion at 2q11.2 Associated with Dysmorphic Features and Developmental Delay S. Sampath, P. Hixson, P. Stankiewicz, S.W. Cheung, P.I. Bader, A. Patel

60 Further Characterization of the Critical Region of the 2p15-p16.1 Microdeletion Syndrome [Withdrawn]

J. Song*, R. Lozano, E. Spiteri, R. Schreck, W.R. Wilcox

*Student presenter

61 Unexpected Abnormal Maternal Karyotype Suggests a Mechanism of Meiotic and Mitotic Errors Resulting in Tetrasomy X in a Patient with Developmental Delay and Dysmorphic Features

F.C. Wheeler, J. Pfotenhauer, J.A. Phillips III 


\section{Non-Human Cytogenetics}

62 Chromosome Abnormalities Identified in a Lowland Gorilla Pedigree

M.L. Houck, S.J. Charter, J.A. Fronczek, M. Domingos, K. Helmick, T. Hermann, G. Mastromonaco, O.A. Ryder, N.C. Hawkes

63 Cytosystematics of African Gerbils (Rodentia, Muridae, Gerbillinae) [Withdrawn] L. Knight

64 Trisomy-Associated Hydrops Fetalis in a Horse Fetus T.L. Lear, J. Lundquist, J.A. Fronczek, S.J. Charter, T.V. Little

65 A Novel Cervid Satellite DNA Originated from a Putative Ancient Centromeric DNA with CDEI-Like Repeated Sequences C.C. Lin, L.-J. Hsieh, Y.-M. Cheng, Y.-C. Li

66 G- and C-Banded Metaphase Karyotypes in the Chinchilla (Chinchilla laniger) H. Pang, S. Li, T. Seale

67 Cytogenetic Analysis of Anuran Species of Akure, Ondo State (South-Western Nigeria) A.A. Sorungbe, I. Awopetu

68 Polymorphism in the Location of Silver-Stained Nucleolus Organizing Regions and the 18S+28S Ribosomal Genes in North American Hylid Frogs [Replaces platform presentation 12]

J.E. Wiley, H.S. Bonner

\section{Cancer Cytogenetics}

69 Investigation of Chromosomal Aberrations in Hepatocellular Carcinoma Patients as Detected by Fluorescence in situ Hybridization

M.A. Al Abboud, M.S. Aly

70 Polysomy 17 as Predictor of HER2 Gene Overexpression in Breast Cancer Patients O. Al-Abdulwahed*, B. McMahill, Y. Zou, R. Saleki

*Student presenter

71 Comparison of Genetic Changes in Transitional and Squamous Bladder Cancers M.S. Aly, H.M. Khaled

72 A t $(8 ; 14)(q 24 ; q 32)$ Translocation Resulting in an Atypical /GH/MYC Genomic Fusion in Chronic Lymphocytic Leukemia P.H. Bui, I. Amarillo, B.S. Shackley, C.A. Tirado

73 A Rare Case of Acute Myeloid Leukemia with an inv(8)(p11.2q13)

C. Henderson, V. McDaniel, D. Graham, K. Maloney, L. Meltesen, X. Liang, K. Swisshelm, L. McGavran

74 Evolution of an Isoderivative Chromosome 7q in t-AML following Treatment of Triple-Negative Breast Cancer S.R. Horn*, K. Nandi, K.L. Deak, C.W. Rehder, B.K. Goodman *Student presenter

75 Correlating Genetic Alterations from Array Comparative Genome Hybridization and Next-Generation Sequencing with Histology and Clinical Outcome in Melanocytic Neoplasms Y.J. Liu, Y. Zhou, E. George

76 Mismatched Crossing-Over between Sister Chromatids Yields Identical Duplication of the Proximal Segment and Deletion of the Distal Segment of IGH Gene but Phenotypic Expression Is Modulated to Mantle Cell Lymphoma or Chronic Lymphocytic Leukemia by the Presence of Additional Chromosome Abnormalities N.S. Mitter

77 Waiting for the Axe to Fall: Ph Chromosome in the Bone Marrow prior to Overt Clinical and Morphologic Evidence of Neoplastic Disease K. Ouyang, M. Jamali, G. Raca, J. Anastasi, O. Borinets, T. Raoul, T. Yessen, V. Aggarwal, J. Shay, C. Rubin

78 MM FISH Panel Validation Findings from Purified Plasma Cells (PPC) D.P. Singh-Kahlon, J. Blackson, S. Ahmad, M. Quiam

79 Multidisciplinary Approach in the Diagnosis of T-PLL P. Starshak, Y. Linhares, P.N. Rao, C.A. Tirado

80 An Unbalanced Rearrangement Involving a Translocation $(6 ; 8)$ in a Case of Diffuse Large B-Cell Lymphoma C.A. Tirado, J.W. Boles, P. Bui, F. Quintero-Rivera

81 Co-Localization and Amplification of MYC and MLL Oncogenes May Suggest a 'Double Hit' Phenomenon in Acute Myeloid Leukemia (AML)

Z.-X. Wang, A. Dulau, J. Gong, S. Peiper, R. Bajaj

82 Optimized Clinical Stratification of Renal Tumors: Validation of SNP Microarray as a First-Tier Genetic Test for Renal Cell Carcinoma

I. Znoyko, M.T. Smith, L.N. Scurry, D.J. Wolff

Preprint Cytogenet Genome Res 136/4/2012

Abstracts will be available online, free of charge 


\section{Chromosomal Breakpoints Target a Variety of Functional Elements in the Human Genome, Including Annotated, Unannotated, Coding and Non-Coding Transcriptional Regions}

N. Tommerup a , I. Bache a , N.M. Nielsen ${ }^{\text {b }}$, S. Kjaergaard ${ }^{c}$, K. Brøndum-Nielsen ${ }^{\mathrm{d}}$, M. Frisch ${ }^{\mathrm{b}}$, A. Lind-Thomsen ${ }^{\mathrm{a}}$, Y. Mang $^{\mathrm{a}}$, C. Hansen ${ }^{\text {a }}$, A. Silahtaroglu a , M. Kirchhoff ${ }^{c}$, P.K.A. Jensen ${ }^{\mathrm{e}}$, C. Fagerberg ${ }^{\text {f }}$ L.N. Krogh ${ }^{\text {g, J. Hansen }}{ }^{\text {h }}$, T. Bryndorfi , Z. El-Schich ${ }^{\mathrm{a}}$, K.F. Henriksen ${ }^{\text {a }}$ C.M. Anderson ${ }^{\text {a }}$, M. Bak ${ }^{\text {a }}$, C. Halgren ${ }^{\text {a }}$

aWilhelm Johannsen Center for Functional Genome Research, Department of Cellular and Molecular Medicine, University of Copenhagen, bepartment of Epidemiology Research, Statens Serum Institut, Copenhagen, 'Department of Clinical Genetics, Rigshospitalet, ${ }^{d}$ Kennedy Center, Glostrup, e Department of Clinical Genetics, Århus University Hospital, Århus, ${ }^{f}$ Department of Clinical Genetics, Vejle Hospital, Vejle, ${ }^{9}$ Department of Clinical Genetics, Odense University Hospital, Odense, hDanish Cytogenetic Central Register, Department of Clinical Genetics, Århus University Hospital, Århus, 'Department of Gynecology and Obstetrics, Hvidovre University Hospital, Copenhagen, Denmark

In the first unbiased long-term clinical follow-up of prenatally diagnosed carriers of de novo balanced chromosomal rearrangements, we observed a significant $(\sim 20 \%)$ morbidity in carriers, a 2-3-fold increase compared to previous estimates. The morbidity exclusively involved neurodevelopmental, cognitive, and psychiatric disorders, indicating that the number of genomic targets for these clinically and genetically overlapping disorders must be numbered in the thousands. By systematic mate-pair sequencing of the cohort, we identified and confirmed some of these targets, including protein coding genes like NPAS3, CELF4 and $A R I D 1 B$, as well as many novel protein-coding candidate disease genes. We observed truncation of evolutionarily conserved putative regulatory gene deserts, suggesting the involvement of longrange position effects, exemplified by truncation of the gene desert distal to the Mowat-Wilson syndrome gene ZEB2. Intriguingly, several breakpoints truncated the novel class of long (intergenic) non-coding RNA genes (lincRNA/lncRNA), exemplified by a breakpoint truncating the canonical lincRNA HOTAIR within the highly conserved HOXC cluster. In addition, breakpoints truncated unannotated transcriptional regions identified by
RNA-Seq of human brain regions, indicating that bioinformatic assessment of chromosomal breakpoints cannot be limited to the known, annotated part of the transcriptome. The translational implication of the study is, that whereas screening for genomic imbalances with array CGH will have limited prognostic value for prenatally diagnosed de novo balanced reciprocal translocations and inversions, next-generation mate-pair sequencing for the first time provides a platform which in many cases will be able to discriminate between rearrangements with low and high morbidity risk. In general terms the study illustrates that a systematic mapping of chromosomal breakpoints by mate-pair sequencing will have the power to saturate all functional compartments of the human genome with mutations.

\section{2 \\ The Impact of Chromosomal Microarray on Clinical Management}

L.B. Henderson ${ }^{\text {a }}$, E. Wohler ${ }^{\text {b }}$, C.D. Applegate ${ }^{\text {a }}$, D.A.S. Batista ${ }^{\mathrm{a}-\mathrm{c}}$

${ }^{a}$ McKusick-Nathans Institute of Genetic Medicine, Johns

Hopkins Medical Institutions, ${ }^{b}$ Cytogenetics Laboratory,

Kennedy Krieger Institute, 'Department of Pathology,

Johns Hopkins University, Baltimore, Md., USA

The clinical utility of chromosomal microarray (CMA) is widely recognized by the genetics community as a useful diagnostic tool. ACMG recommends CMA as a first-line test to evaluate individuals with intellectual disabilities and anomalies. Despite this, some payers have been reluctant to reimburse this test stating that it lacks clinical utility. We aimed to determine the direct impact CMA results have on clinical management.

We reviewed 1,360 cases submitted to our laboratory for CMA analysis. 190 cases $(14.0 \%)$ had copy number variations (CNVs) reported as pathogenic, and $184(13.5 \%)$ had CNVs of uncertain clinical significance. In our initial analysis of the pathogenic CNVs, 14 (7.4\%) results triggered clinical action or confirmed a diagnosis with clinical implications. Seven patients had abnormalities of a cancer predisposition gene (3 APC, 3 VHL, 1 NF2). Detection of these alterations resulted in recommendations for appropriate cancer surveillance. In 1 case, CMA refined the breakpoints of a known deletion, excluding the presence of the $A P C$ gene, and thus eliminating unnecessary cancer surveillance and optimizing allocation of resources. Other medically relevant

\section{KARGER}

Fax +4161306 1234

E-Mail karger@karger.ch

www.karger.com
(C) 2012 S. Karger AG, Basel

$1424-8581 / 12 / 0000-0000 / \$ 38.00 / 0$

Accessible online at:

www.karger.com/cgr 
findings included intragenic $D M D$ deletions in 3 pre-symptomatic boys, and a homozygous deletion of CTNS in a girl with suspected cystinosis. CMA results led to change in medication management in a seizing patient and another with dystonia.

CMA findings in a proband virtually always have clinical significance for other family members. Almost all pathogenic results refine the recurrence risk and allow for accurate genetic counseling. These results may also prompt similar clinical action in relatives harboring the same abnormality. Importantly, these diagnoses could not have been made using only traditional cytogenetic methods that are typically covered by insurance. Our results demonstrate that CMA has a direct impact on clinical management in a significant portion of patients tested and support the need for its coverage by health insurance.

\section{3 \\ Microarray Analysis of X Chromosome Aberrations in 45 Women with Highly Skewed $X$ Inactivation and 45 Controls}

V. Jobanputra ${ }^{\text {a }}$ B. Levy ${ }^{\text {a }}$, A. Kinney ${ }^{\text {b }}$, S. Brown ${ }^{c}$, M. Shirazi ${ }^{\text {a }}$, C.-Y. Y $u^{\text {d }}$, J. Kline ${ }^{\text {b, e }}$, D. Warburton ${ }^{d}$

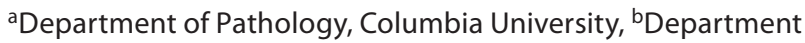
of Epidemiology, Mailman School of Public Health, Columbia University, New York, N.Y., 'Department of Obstetrics, Gynecology and Reproductive Science, The University of Vermont, Burlington, Vt., ${ }^{d}$ Departments of Genetics and Development, and Pediatrics, Columbia University, ' Sergievsky Center, Columbia University and New York State Psychiatric Institute, New York, N.Y., USA

X chromosome inactivation (XCI) occurs in early embryogenesis. Since inactivation occurs randomly between the two X chromosomes, in theory, $50 \%$ of cells will contain an active maternally inherited X chromosome and $50 \%$ of cells will contain an active paternally inherited X chromosome. Highly skewed XCI (HSXI) can occur by chance but is thought to result most often from selection in a population of cells in which inactivation was initially random. The degree of selection is known to vary among tissue types. HSXI often occurs in women with a structurally abnormal X chromosome (deletion or translocation) in a manner that preserves the normal X chromosome and autosomal dosage.

High-resolution microarrays allow detection of copy number changes (CNCs) below the resolution of chromosome preparations. We hypothesized that small CNCs might be responsible for HSXI in women with normal karyotypes. To examine this question we performed a SNP microarray analysis using the Affymetrix 6.0 platform on 45 women with HSXI $\geq 85 \%$ in a blood sample and 45 control women without skewing. We analyze CNCs confirmed by FISH or qPCR.

Cases and controls did not differ in the frequency of $\mathrm{X}$ chromosome CNCs $\geq 100 \mathrm{~kb}$ nor in the frequency of CNCs that contained genes. One woman with HSXI $>90 \%$ in blood as well as left- and right-side buccal smears had a 5.5-Mb deletion in Xp22.2p22.1. This gene-rich deletion could affect the viability of male pregnancies; it also appears to be associated with minor features of Nance-Horan in female carriers in the family.
Our study shows that highly skewed X inactivation found only in a blood sample is rarely due to X chromosome CNCs detectable by microarray. This result goes against the prevailing view that cryptic changes in the $\mathrm{X}$ chromosome are a common cause of HXSI.

4

Weird Animal Genomes, Sex and the Future of Men

J.A.M. Graves

The Australian National University, Canberra, A.C.T., Australia

The human $\mathrm{X}$ is a middle-sized chromosome, rich in 'brainsand-balls' genes involved in reproduction and intelligence (often both), and thought to have had a major role in human evolution. The tiny $\mathrm{Y}$ is a genetic wasteland - full of genetic junk and bearing only 45 genes, most of which are active only in testis.

Human sex chromosomes are nothing but trouble. The $\mathrm{X}$ and $Y$ don't pair very well at male meiosis (causing infertility), the dosage difference of the $\mathrm{X}$ between the sexes requires compensation, and its monosomic state in males causes sex-linked diseases, and translocations of the terminal SRY lead to sex reversal. Are they weird because they work better, or is it some horrible evolutionary accident?

To follow the evolution of the human $\mathrm{X}$ and $\mathrm{Y}$, we compare the chromosomes, genes and DNA in distantly related mammals and even birds and reptiles. The genomes of Australia's unique kangaroos and platypus, now completely sequenced, are a goldmine of new information. Kangaroo sex chromosomes reveal the original mammal sex chromosomes, while the bizarre platypus sex chromosomes (more related to those of birds) tell us that human sex chromosomes are relatively young. Reptiles display all kinds of sex chromosome systems as well as temperature-dependent sex, and reveal that the function of triggering sex determination has been taken on by different regions of the very conserved amniote genome. The human X and Y evolved from an ordinary autosome pair about 166 million years ago as the $Y$ degraded progressively.

At this rate of degradation, the human Y chromosome will disappear in just 5 million years. If humans don't become extinct, new sex determining genes and chromosomes will evolve, maybe leading to the evolution of new hominid species.

\section{5}

\section{Utility of SNP Arrays in Detecting, Quantifying, and Determining Meiotic Origin of Tetrasomy $12 p$ in Blood from Pallister-Killian Patients}

\author{
L.K. Conlin ${ }^{\text {a }}$, K. Izumi ${ }^{\text {b }}$, M. Kaur ${ }^{\text {b }}$, D. Clark ${ }^{\text {b }}$, A. Wilkens ${ }^{\text {b }}$, E. Zackai ${ }^{\text {b }}$, \\ M.A. Deardorff ${ }^{\mathrm{b}}$, N.B. Spinner ${ }^{\mathrm{a}}$, I.D. Krantz ${ }^{\mathrm{b}}$ \\ ${ }^{a}$ Department of Pathology and Laboratory Medicine, \\ ${ }^{b}$ Department of Pediatrics, The Children's Hospital of \\ Philadelphia and University of Pennsylvania School of Medicine, \\ Philadelphia, Pa., USA
}

Pallister-Killian syndrome is a rare syndrome associated with the presence of an isochromosome $12 \mathrm{p}, \mathrm{i}(12 \mathrm{p})$. The cytogenetic diagnosis is complicated by the low frequency of this supernu-

Preprint Cytogenet Genome Res 136/4/2012 
merary chromosome in PHA-stimulated peripheral blood lymphocytes, which requires cytogenetic analysis of fibroblast cells for diagnosis. We studied 23 patients with a previous clinical and/ or cytogenetic diagnosis of Pallister-Killian syndrome using the Illumina Quad610 SNP array to investigate our ability to identify $\mathrm{i}(12 \mathrm{p})$ in blood and fibroblasts. For this study, patient age at the time of sample collection was between 2 months to 19 years. Tetrasomy $12 \mathrm{p}$ was identifiable by the array analysis in all fibroblast samples; however, it was detected in only half of blood samples. The genotyping information from the SNP arrays allowed for the detection of at least $5 \%$ mosaicism. The genotyping pattern suggested meiosis II origin for the majority of patients, with 3 genotypes at the $12 \mathrm{p}$ terminus and 2 genotypes present near the centromere. Locations of recombinations showed a hotspot around basepair position $17 \mathrm{Mb}$ (hg18). No patients demonstrated isodisomy of the long arm of chromosome 12. Taken together, these findings suggest that the origin of the $\mathrm{i}(12 \mathrm{p})$ was a centromeric misdivision in meiosis II, after crossing-over took place in meiosis I. Three patients had unusual structural findings not detectable by array, with 1 patient with hexasomy $12 \mathrm{p}, 1$ patient with trisomy $12 \mathrm{p}$, and 1 patient with a derivative $12 \mathrm{p}$ containing tandem copies of $12 \mathrm{p}$. Analysis of the percentage of abnormal cells with patient age suggests that the frequency of the $i(12 p)$ decreased with age in blood, but not in fibroblasts; however, the percent of $i(12 p)$ decreased in vitro with fibroblast passage. These findings stress the importance of traditional cytogenetic techniques, in addition to array technology, in the diagnosis of Pallister-Killian syndrome.

\section{6 \\ Small Recurrent Deletions and Reciprocal Duplications in 2q21.1, Including Brain- Specific ARHGEF4 and GPR148, in Patients with Developmental Delay, ADHD, Autism, and Epilepsy}

\begin{abstract}
A.V.Dharmadhikari ${ }^{\text {a }}$, S.-H.L. Kang ${ }^{\text {a }}$, P. Szafranski ${ }^{a}$, R.E. Person ${ }^{\text {a }}$, S. Sampath ${ }^{\text {a }}$ S.K. Prakash ${ }^{\text {b }, ~ P . I . ~ B a d e r ~}{ }^{\text {, J.A. Phillips III }}$, V. Hannig ${ }^{\mathrm{c}}$, M. Williams ${ }^{c}$, S.S. Vinson ${ }^{\mathrm{e}}$, A.A. Wilfong ${ }^{\mathrm{f}}$, W.J. Craigen ${ }^{\mathrm{a}}$, J.Wiszniewska ${ }^{\text {a }, A . P a t e l}{ }^{\text {a }}$,W. Bi ${ }^{\text {a }}$, J.R. Lupski ${ }^{\text {a, g, h, J. Belmont }}$, S.W. Cheung ${ }^{a}$, P. Stankiewicz ${ }^{\text {a }}$

aDepartment of Molecular \& Human Genetics, Baylor College of Medicine, ${ }^{b}$ Department of Internal Medicine, University of Texas Health Science Center at Houston, Houston, Tex., 'Division of Medical Genetics and Genomic Medicine, Vanderbilt University School of Medicine, Nashville, Tenn., dNortheast Indiana Genetic Counseling Center, Fort Wayne, Ind., 'Developmental Pediatrics, fPediatric Neurology, Texas Children's Hospital, Baylor College of Medicine, 9Department of Pediatrics, Baylor College of Medicine, hTexas Children's Hospital, Houston, Tex., USA
\end{abstract}

Attention-deficit hyperactivity disorder (ADHD) is a neurodevelopmental disorder in children and adults characterized by inattentive, hyperactive, and impulsive behaviors that may lead to significant psychosocial impairments. Genome-wide association and linkage studies identified several candidate loci and genes, and both recurrent and nonrecurrent copy-number variants (CNVs) have been reported. We have identified an $\sim 450-\mathrm{kb}$ (unique sequence) recurrent deletion in a previously linked locus at 2q21.1 in 5 unrelated families with ADHD, epilepsy, and other neurobehavioral abnormalities from 10,360 samples referred for clinical chromosomal microarray analysis. Additionally, DECIPHER (http://decipher.sanger.ac.uk) patient 2311 was found to have the same deletion and presented with aggressive behavior. The deletion was not found in 6 control groups consisting of 14,000 healthy individuals. The deletion is flanked by large, complex low-copy repeats with directly oriented subunits of $\sim 109 \mathrm{~kb}$ in size that have $97.7 \%$ DNA sequence identity. We sequenced the deletion breakpoints within the directly oriented paralogous subunits of the flanking low-copy repeat clusters, demonstrating nonallelic homologous recombination (NAHR) as a mechanism of formation. We have also identified reciprocal duplications in 5 unrelated families with autism, developmental delay, seizures, and ADHD. The rearranged segment harbors 5 genes: GPR148, FAM123C, ARHGEF4, FAM168B, and PLEKHB2. Expression of ARHGEF4 (Rho guanine nucleotide exchange factor 4; also called ASEF) is restricted to the brain and may regulate the actin cytoskeletal network, cell morphology and migration, and neuronal function. GPR148 encodes a G protein coupled receptor protein expressed in the brain and testes. We suggest that small recurrent deletions and reciprocal duplications in 2q21.1 are pathogenic for developmental delay, ADHD, epilepsy, and neurobehavioral abnormalities and, because of their small sizes and more severe phenotypes, might have been missed in other previous genome-wide ADHD screening studies using SNP analyses.
7

\section{Next Generation Approaches to the Analyses of} Inversions

S.M. Bailey, E. Zimmerman, M.N. Cornforth, J.S. Bedford,

E.H. Goodwin, F.A. Ray

Department of Environmental \& Radiological Health Sciences, Colorado State University, and KromaTiD, Inc., Fort Collins, Colo., USA

Of all possible types of structural chromosomal anomalies, inversions - a reversal of orientation of material within a chromosome - are perhaps the most difficult to detect. This is especially true of small inversions, most of which are invisible to current cytogenetic techniques. Their small size, however, does not preclude them from being any less detrimental than other, more easily visible rearrangements, such as translocations. This is because inversions form through the same mechanisms as translocations, and should have similar untoward genetic effects, such as disruption of regulatory sequences that control gene expression, or creating genetic chimeras like the $B C R-A B L$ gene fusion. Moreover, it is likely that small inversions are among the most transmissible forms of chromosomal damage, a feature that lends credence to the concept that many cytogenetically invisible (cryptic) rearrangements of this type could contribute significantly to a variety of disease states, including carcinogenesis.

Various monochromatic chromosome banding techniques (e.g. Q-, G-, and R-banding) have been used to detect inversions and continue to be the most common approach for clinical cyto- 
genetics. However, such banding techniques suffer severe limitations in terms of resolution, and they require considerable time and expertise to interpret. Today's sophisticated mBAND technology offers improvement, but remains restricted to the resolution of banding patterns and limited to analysis of one chromosome at a time.

Whole chromosome paint (WCP) and mFISH/SKY (multicolor) technologies have revealed the previously unimaginable complexity of interchromosomal rearrangements. There is every reason to believe that, just as for complex chromosome aberration formation between different chromosomes, improved resolution for detecting rearrangements occurring within chromosomes will open our eyes to their frequency and complexity. Here, we demonstrate the feasibility of a novel approach based on the strandspecific technique of chromosome orientation FISH (CO-FISH) for the purpose of exploring its potential utility in high-resolution inversion detection.

\section{8 \\ De novo Copy Number Losses of Chromosomes 2q22.3q23.2 and 5q11.2q12.2 in a Female Patient with Clinical Features of Angelman Syndrome: A Case Report}

\section{A.D. Chaubey, F. Bartel, S.A. Skinner, B.R. DuPont}

Greenwood Genetic Center, Greenwood, S.C., USA

Patients with Angelman syndrome present with intellectual disability, ataxia, severe language and speech delays, seizures, and microcephaly. Here we report a 20 -year-old female with significant developmental delay, expressive language disorder, seizures and unique behavioral features which are suggestive of Angelman syndrome. The patient has been followed at the Greenwood Genetic Center since 14 months of age when she presented with developmental delay. At 3 years of age, she developed seizures. Prior genetic studies including chromosome analysis, fragile- $\mathrm{X}$ testing, methylation for the Prader-Willi/Angelman syndrome, UBE3A and $M E C P 2$ gene sequencing and a BAC array were all negative. Subsequently, high resolution SNP microarray analysis was performed using the Genome-wide SNP 6.0 Microarray system from Affymetrix, Inc. (Santa Clara, USA) and revealed 2 significant copy number losses: (1) $1.8 \mathrm{Mb}$ deletion of chromosome 2q22.3q23.2 (hg18: 148,306,196-150,165,870), including the $M B D 5$ and $O R C 4 L$ genes. Recent reports have documented haploinsufficiency of $2 \mathrm{q} 23.2$ with the critical gene being MBD5 in individuals with microcephaly, intellectual disabilities, severe speech impairment, and seizures. Mutations of the ORC4L gene have been associated with Meier-Gorlin syndrome-2 form of recessive microcephalic primordial dwarfism. (2) $5.0 \mathrm{Mb}$ deletion of chromosome 5q11.2q12.2 (hg18: 58,366,026-63,398,056), including PDE4D, ERCC8 and NDUFA12L genes, which may contribute towards some of the clinical features observed in this patient. Mutations of the ERCC8 gene have been associated with Cockayne syndrome type $\mathrm{A}$, which is a recessive syndrome characterized by severe progressive neurologic degeneration resulting in intellectual disability. Though parental studies revealed both of these deletions to be of de novo origin, there is an increased likelihood that deletion of $2 \mathrm{q}$ contributes towards the majority of features observed in this patient. This case report highlights the impact of high resolution microarray analysis on clinical cytogenetics by correlating varying clinical features with the specific genes within the aberrations.

\section{9 \\ Maternal Uniparental Disomy of Chromosome 7 in a Patient with Myoclonus-Dystonia}

\author{
M.B. Sheridan a, A.B. Telegrafi a , V. Stinnett ${ }^{\text {b }}$, T.M. Dawson ${ }^{c}$, \\ J. Bodurtha a , D.A.S. Batista ${ }^{\mathrm{a}} \mathrm{b}, \mathrm{d}$ \\ a McKusick-Nathans Institute of Genetic Medicine, Johns \\ Hopkins University School of Medicine, ${ }^{b}$ Cytogenetics \\ Laboratory, Kennedy Krieger Institute, Institute for Cell \\ Engineering, Departments of Neurology and Neuroscience, \\ Johns Hopkins University School of Medicine, dDepartment of \\ Pathology, Johns Hopkins University, Baltimore, Md., USA
}

Myoclonus-dystonia (MD) is a movement disorder characterized by sudden, brief involuntary muscle jerks and contractions causing abnormal postures. Mutations in epsilon sarcoglycan (SGCE), a maternally imprinted gene at $7 \mathrm{q} 21.3$, are associated with MD. We report a 24 -year-old male with short stature $(151.9 \mathrm{~cm}$; $<5 \%)$ evaluated for jerking of the arms and shoulders with unknown etiology. SNP array (Illumina 1M-Quad) did not identify significant copy number changes, but revealed 3 long continuous stretches of homozygosity on chromosome 7. The presence of homozygous regions exclusively on one chromosome suggested uniparental isodisomy (iUPD). Because SNP arrays can only detect isodisomy, we hypothesized that the remainder of chromosome 7 was heterodisomic, implying the occurrence of 5 meiotic crossover events in one of the parents. Parental SNP arrays (Illumina $300 \mathrm{~K})$ revealed maternal UPD7. The mechanism for UPD7 is hypothesized to be trisomy 7 rescue with loss of the paternal homolog. The high number of cross-over events and relative proximity to the centromere may have led to entanglement of the maternal meiotic bivalent resulting in nondisjunction or precocious separation of sister chromatids. Maternal UPD7 is found in approximately $10 \%$ of patients with Silver-Russell Syndrome (SRS), a condition characterized by prenatal and postnatal growth retardation. Recently, 5 patients with SRS and maternal UPD7 were identified with movement disorders. This report adds further evidence that loss of the paternally expressed SGCE is the cause of MD in these patients. Although SRS was not initially considered in our patient, our findings provide a unifying diagnosis for his short stature and MD and will help to optimize his medication regimen. Additionally, we provide mechanistic insights to the origin of aneuploidy/UPD and evidence that the presence of MD differentiates SRS due to maternal UPD7 and other etiologies. 


\section{0}

\section{A Four Generation Family with Duplication of 17p13.3 with Variable Split-Hand/Foot Malformation}

B.R.DuPont ${ }^{\text {a }}$, A.D. Chaubey ${ }^{\text {, }}$, F.O. Bartel ${ }^{\text {a }}$, K.R. Holden ${ }^{\text {a }}$,

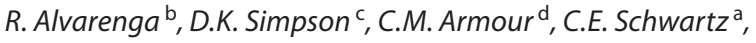
D.B. Everman ${ }^{\mathrm{a}}$

${ }^{\mathrm{a} G r e e n w o o d ~ G e n e t i c ~ C e n t e r, ~ G r e e n w o o d, ~ S . C ., ~ U S A ; ~}{ }^{\mathrm{b}}$ Hospital

Escuela Materno-Infantil, University of Honduras Medical

School, Tegucigalpa, Honduras; ${ }^{\circ}$ Oregon Health Sciences

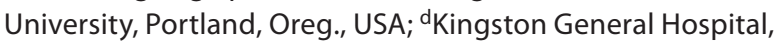

Kingston, Ont., Canada

Split-hand/foot malformation (SHFM) is a congenital limb malformation characterized by median clefts of the hands and/ or feet with underdevelopment or absence of phalanges and metacarpal/metatarsal bones and syndactyly of the remaining digits. Isolated SHFM and various syndromic forms have been reported, with significant genetic heterogeneity. Some forms of SHFM are autosomal dominant with highly variable expressivity and incomplete penetrance, and their underlying genetic causes can include single gene mutations and balanced or unbalanced chromosome rearrangements. We recently reported microduplications of 17p13.3 in 3 unrelated families with SHFM and long bone deficiency (SHFLD). These duplications are associated with incomplete penetrance and have defined a $173 \mathrm{~kb}$ critical region which includes 2 genes, $A B R$ and $B H L H A 9$. Additional screening of 30 patients with either SHFM or SHFLD identified 3 patients with 17p13.3 duplications and narrowed our SHFM/SHFLD critical region to $135 \mathrm{~kb}$. We have been able to extend the molecular analysis of one of the familial cases to include 21 family members from 4 generations and correlate these findings with the presence and severity of limb involvement. Eight family members were identified as being affected and having the $17 \mathrm{p} 13.3$ duplication, including 2 with SHFLD and 6 with only hand/foot involvement. Five family members, only 1 of whom was known to be an obligate carrier, did not have overt signs of SHFM but were found to carry the duplication. These results underscore the highly variable expressivity of the SHFM and SHFLD phenotypes related to 17 p13.3 duplications and indicate that such duplications are a relatively frequent cause that should be sought with SNP-based microarray analysis. Moreover, they highlight the importance of considering incomplete penetrance in the context of genetic counseling and molecular testing for unaffected at-risk family members.

\section{1}

Development and Application of Molecular Tools for Camelid Cytogenetics

\author{
F. Avila ${ }^{\text {a }}$ P.J. Das a , M. Kutzler ${ }^{\text {c }}$ E. Owens ${ }^{\text {b }}$, P. Perelman ${ }^{\text {d, }}$ \\ T. Raudsepp ${ }^{\mathrm{a}}$
}

Departments of aeterinary Integrative Biosciences and beterinary Pathobiology, Texas A\&M University, College Station, Tex., 'Department of Animal Sciences, College of Agricultural Sciences, Oregon State University, Corvallis, Oreg., 'Laboratory of Genomic Diversity, National Cancer Institute,

Frederick, Md., USA

The development of genome maps constitutes a key feature for understanding the architecture and comparative evolution of genomes. Some livestock species such as cattle, horses and pigs have received considerable attention over the years due to their economic importance. In contrast, even though alpacas and llamas are gaining popularity as production and companion animals, camelid cytogenetics and genome mapping lag far behind those of other domesticated species.

One of the reasons for the scarce body of knowledge regarding the camelid genome is their particularly difficult karyotype. All 6 extant camelid species have a diploid number of $2 n=74$; the gross morphological similarity shared by many of the autosomes, combined with the relatively small size of some chromosome pairs, present serious challenges for identifying individual chromosomes using conventional cytogenetic techniques. Our primary goal was to develop a set of molecular markers that can be used for the unambiguous identification of individual camelid chromosomes. For this, we isolated 42 gene-containing BACs and used them as FISH probes for 35 autosomes and the X chromosome. The selected markers included candidate genes for congenital disorders and phenotypic traits.

This collection of markers is part of our ongoing efforts to construct a whole genome cytogenetic map for the alpaca, the first for any camelid species. The map is needed to effectively anchor the radiation hybrid map and genome sequence assembly to physical chromosomes. The set of chromosome-specific markers is also a critical tool for camelid clinical cytogenetics, aiding in the identification of individual chromosomes, as well as structural aberrations. Markers developed in this study were recently used to identify the chromosomes involved in an autosomal translocation in a sterile male llama.

\section{2 [Withdrawn] \\ Noncoding RNAs and Chromosome Change \\ R.J. O'Neill \\ University of Connecticut, Storrs, Conn., USA}

Genetic lesions that contribute to the progression of cancer from a primary tumor to metastases often involve a complex process of chromosome evolution, either progressive or catastrophic; however, the primary factor(s) that precede(s) complex rearrangements remain largely unknown. As a model to investigate the mechanisms that drive metastatic potential and chromosome evo- 
lution in tumorigenesis, an inbred line of Peromyscus leucopus was isolated that carries a high frequency of malignant Harderian gland tumor formation that is immediately and predictably metastatic and invades the lymphatic system, lungs and liver. Employing chromosome microdissection and deep-sequencing, we have identified the primary lesion that contributes to both tumor susceptibility and metastasis in this susceptible mouse strain. Following a period of high transcriptional activity restricted to the cancer-susceptible strain prior to tumor onset, a noncoding RNA is amplified in the primary tumor and becomes a platform that mediates heterogeneous rearrangements and drives karyotypic evolution. The instability of this noncoding RNA is the only lesion observed in metastases, likewise acting as a platform for large-scale chromosome rearrangements. This study demonstrates a mechanistic link between the derivation of distinct chromosomal aberrations in a primary tumor and those in secondary metastases and, taken with our work on noncoding RNAs and centromere function, highlights the role noncoding RNAs can play in chromosome instability.

\section{3}

\section{Identification and Expression Analysis of the Pig Pseudoautosomal Region Using Molecular Cytogenetics Approach}

\section{P.J. Das, D.K. Mishra, F. Avila, G. Johnson, B.P. Chowdhary, T. Raudsepp}

Department of Veterinary Integrative Biosciences, College of Veterinary Medicine and Biomedical Sciences, Texas A\&M University, College Station, Tex., USA

The mammalian pseudoautosomal region (PAR) is essential for sex chromosome segregation in male meiosis but might also be involved in other biological functions. Besides human and mouse, comprehensive information about the organization of the PAR is available only for horses, cattle and dogs. Notably, all 5 PARs differ in size and gene content, while almost no information is available about the functions of the PAR genes in any species.

Here we characterized the PAR in pigs, and determined expression levels of selected PAR genes at different developmental stages of pig embryos and placenta. We isolated $20 \mathrm{BAC}$ clones containing 14 putative PAR genes. The clones were FISH mapped to male pig metaphase chromosomes to determine their PAR origin. All 14 genes (ARSD,E,F,H, CRLF2, CSRF2RA, GPR143, HDHD1, KAL1, MXRA5, PNPLA4, PRKXY, SHROOM2, $S L C 25 A 6)$ were assigned to the pig PAR because signals were detected both on X and Y. However, a BAC clone proximal to SHROOM2 mapped to the $\mathrm{X}$, indicating the likely location of the PAR boundary within a $500 \mathrm{~kb}$ segment at $5.2 \mathrm{Mb}$ in the sequence map. The results were validated by quantitative PCR showing that male-to-female copy number ratio is 1 for the PAR, and 0.5 for Xspecific sequences. Expression analysis of 10 PAR and $2 \mathrm{X}$ genes in placenta and embryos revealed upregulation of the PAR genes in placenta compared to the embryo.

We conclude that the pig PAR is similar in size and gene content to the cattle PAR, but is larger than the PAR in humans or horses. Upregulation of the PAR genes in placenta underlines their important role in early development and might explain why sex chromo- some aneuploidies, such as X-monosomy, are better tolerated in species with small PARs (human and horse) but seriously affect embryonic survival in species with large PARs (cattle and pigs).

\section{4}

\section{Devil Facial Tumour Disease: Does Chromosome 1 Hold the Key?}

\section{J.E. Deakin, Y. Zhang}

Research School of Biology, The Australian National University, Canberra, A.C.T., Australia

A fatal contagious cancer, known as devil facial tumor disease (DFTD) is driving the iconic Tasmanian devil to extinction. The infectious agent in DFTD is the tumor itself, which is transmitted as an allograft by biting. The original DFTD tumor arose over 15 years ago in the Schwann cell of a female devil. Despite passage through over 100,000 individuals, DFTD chromosomes have remained remarkably stable. Cytogenetic gene mapping suggested that rearrangement of chromosome 1, perhaps through a chromothripsis event, may have been the initial step in the development of DFTD and that the maintenance of the arrangement of this chromosome 1 material in the tumor is critical for the survival of DFTD. Karyotypic differences identified between different DFTD subclones by chromosome painting and gene mapping were restricted to well demarcated regions of the genome, predominantly consisting of chromosome 4, 5 and X material but not chromosome 1 .

The long-term stability of DFTD tumor chromosomes indicates that DFTD does not share the overt chromosome instability observed for many solid tumors in humans, raising the question as to whether selection is acting on DFTD chromosome 1 material to maintain the tumorigenic properties of a DFTD cell. To test this hypothesis, we have constructed a dense gene map of devil chromosome 1 and determined the location of these genes in 3 different DFTD subclones. Mapping data confirms the predicted stable nature of most chromosome 1 material in DFTD. Our current efforts are focused on mapping candidate genes from chromosomes 1 that may play a role in DFTD tumorigenesis. This research will help us to determine whether chromosome 1 holds the key to understanding DFTD.

\section{5}

\section{Array CGH Studies in Equine Disorders of Sexual Development - SRY-Positive Sex Reversal and Cryptorchidism}

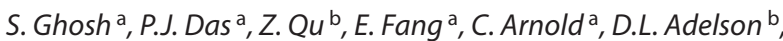
B.P. Chowdhary ${ }^{\mathrm{a}}$, T. Raudsepp ${ }^{\mathrm{a}}$

TTexas A\&M University, College Station, Tex., USA;

${ }^{b}$ Centre for Bioinformatics and Computational Genetics, University of Adelaide, Adelaide, S.A., Australia

Genomic copy number variants (CNVs) are an important source of genetic diversity and play a role in complex diseases and congenital disorders, including disorders of sexual development 
(DSDs). Here we used an equine whole genome 400K tiling array by comparative genomic hybridization (CGH) to investigate the involvement of CNVs in equine DSDs.

The sex reversal females ( $\mathrm{n}=4$; Appaloosa, Quarter Horse, 2 Standardbred) had normal male 64,XY karyotypes with an intact $S R Y$ gene. At conservative CNV calling criteria ( $\geq 0.5 \log _{2}$ ratio over 5 probes) 187 CNVs were detected (126 gains, 61 losses) which varied in numbers, locations and size between the individuals. Among shared CNVs, the most functionally relevant was a $\sim 300 \mathrm{~kb}$ deletion in 2 Standardbreds on ECA29 involving aldoketo reductase gene cluster known to catalyze the conversion of sex hormones. The deletion was validated by PCR and FISH and is the first evidence about a likely molecular component of SRYpositive sex reversal in horses.

Concurrently, CNV studies were initiated for cryptorchidism which is a complex disorder with poorly understood genetic component, and affects about $8 \%$ of live male births both in horses and humans. Array $\mathrm{CGH}$ was conducted in 3 bilateral abdominal cryptorchids (2 American Quarter Horses and a pony) yielding in 104 CNVs. The results were aligned with the current CNV map of the horse genome (based on array CGH analysis of 26 normal horses from 13 diverse breeds), and $2 \mathrm{CNV}$ regions were considered cryptorchid specific. Notably, in 2 cryptorchids we found the same massive deletion in aldo-keto reductase gene cluster as in $S R Y$-positive sex reversals, suggesting that the 2 conditions might share common genetic alterations.

These pilot studies effectively validate the utility of the tiling array and suggest the involvement of CNVs in the genetics of equine DSDs.

\section{6 \\ Dicentric Chromosomes Are Frequent in Chronic Lymphocytic Leukemia (CLL)

\author{
N.A. Heerema ${ }^{\text {a }}$, H. Breidenbach ${ }^{\text {a }}$, C. Miller ${ }^{\text {a }}$, A.McFaddin $^{\text {a }}$, \\ S. Haudenshield ${ }^{a}$, N. Muthusamy ${ }^{\text {b }}$, J.C. Byrd ${ }^{\mathrm{b}}$ \\ Departments of a Pathology and ${ }^{\mathrm{b} H e m a t o l o g y}$, The Ohio State \\ University, Columbus, Ohio, USA
}

Dicentric chromosomes (dic) are rare, but recurrent abnormalities in hematologic malignancies. In myeloid leukemias, dic occur infrequently, although $\mathrm{i}(17 \mathrm{p})$ is recurrent in both AML and accelerated/blast phase CML. Dicentrics with chromosome 9 p-arm breakpoints are relatively common in acute lymphoblastic leukemia. We previously reported a recurrent dic $(17 ; 18)$ in CLL. We identified an additional 130 dicentrics in 93 of 971 (9.6\%) CLL patients. Sixty-one patients had one dicentric, 25 had two, 5 had three, and 1 had four. All chromosomes except the $\mathrm{Y}$ were involved in at least 1 dicentric. The most common dicentrics were $\operatorname{dic}(17 ; 18)$ (p11.2;p11.2), dic(8;17)(p11.2;p11.2), dic(17;20)(p11.2;p11.2), dic(17; 21)(p11.2;p11.2) and $\operatorname{dic}(4 ; 17)(\mathrm{p} 11.2 ; \mathrm{p} 11.2)$ in $19,13,6,5$ and 5 patients, respectively. All but 3 recurrent dic had a $17 \mathrm{p} 11.2$ breakpoint, and $62(66.7 \%)$ cases had at least $1 \mathrm{dic}(\mathrm{V} ; 17)$. There were 54 different dic not involving chromosome 17. Dic $(14 ; 15)$, dic $(14 ; 18)$, and $\operatorname{dic}(15 ; 18)$ occurred in 4,4 and 3 cases. The $\mathrm{p}$-arms of acrocentric chromosomes occurred in 26 different dic, 4 different 'Robertsonians' in 9 patients. $14 \mathrm{p}$ was involved in 19 cases in 9 different dic. Twenty-three breakpoints were centromeric, 16 telomeric, and 14 were along the chromosome arms. Most cases had complex karyotypes, only 6 had a sole dic; 78 (83.9\%) cases had $\geq 3$ abnormalities, the CLL definition of complexity, and 65 (69.9\%) had $\geq 5$ abnormalities. Only 18 cases had normal TP53 interphase FISH; 4 of these had a low frequency of metaphases with del(17p), 2 confirmed by TP53 FISH. Eight TP53 deleted cases had 17p abnormalities other than $\operatorname{dic}(\mathrm{V} ; 17)$. In conclusion, dicentric chromosomes are very common in CLL and involve most chromosomes. Some dic are common, with $\operatorname{dic}(\mathrm{V} ; 17)$ exceptionally frequent; others are unique. The reason for this high frequency of dicentric chromosomes and their clinical significance in CLL are unknown.

\section{7 \\ Diagnostic Application of High Resolution SNP Arrays for Children with Brain Tumors}

J.J. Roth ${ }^{\mathrm{a}}$, M.J.Dougherty ${ }^{\mathrm{C}}$, L.S. Tooke ${ }^{\mathrm{b}}$, A.F. Teplick ${ }^{\mathrm{a}}$, J.A. Biegel ${ }^{\mathrm{a}, \mathrm{b}, \mathrm{d}}$

Departments of apediatrics and ${ }^{b}$ Pathology and Laboratory Medicine, The Children's Hospital of Philadelphia, Philadelphia, Pa., 'Penn State Hershey College of Medicine, Hershey, Pa.,

dPerelman School of Medicine at the University of Pennsylvania, Philadelphia, Pa., USA

The clinical utility of high resolution SNP arrays for pediatric hematologic malignancies and solid tumors has been previously demonstrated by our laboratory. Since the introduction of this test in 2008 , we analyzed over 35 brain tumor specimens using the Illumina Human610-Quad or HumanOmni1-Quad Bead Chip arrays.

Medulloblastoma $(\mathrm{MB})$ and primitive neuroectodermal tumors comprised approximately one third of the tumors analyzed. We identified a variety of abnormalities including monosomy 6 , deletion of $9 \mathrm{q}$, and isochromosome $17 \mathrm{q}$ with and without $M Y C$ amplification. These abnormalities allow further classification of the MBs into 1 of the 4 diagnostic and prognostic molecular subgroups: WNT group, SHH group, groups 3 and 4 . Additionally, we were able to resolve the diagnosis of 1 tumor that was thought to be a rhabdoid tumor. Array findings on this tumor revealed the presence of an isochromosome $17 \mathrm{q}$ and $M Y C$ amplification, thus classifying it as a MB. The high grade gliomas included anaplastic oligoastrocytoma, anaplastic oligodendroglioma, anaplastic astrocytoma and glioblastoma. Array results on these tumors revealed multiple chromosomal abnormalities including homozygous deletions of CDKN2A and PTEN in addition to whole chromosome duplications and deletions. The low grade gliomas represent the largest sample of tumors that we have analyzed and include juvenile pilocytic astrocytomas (JPA), fibrillary astrocytomas, pleomorphic xanthoastrocytomas (PXA), pilomyxoid astrocytomas, ependymomas, and gangliogliomas (GG). Whole chromosome copy number gains were seen in GG, fibrillary astrocytoma and PXA. Homozygous CDKN2A deletions were also seen in some GG and PXA samples. We identified $1 \mathrm{GG}$ and 1 fibrillary astrocytoma that appear to have novel $B R A F$ deletions, supporting the fact that $B R A F$ alterations are not specific for JPAs. In summary, high resolution SNP arrays are a powerful platform for identifying tumorassociated chromosomal alterations and are an important adjunct to histology for the diagnosis of children with brain tumors. 


\section{8 \\ Metaphase Induction in Multiple Myeloma: A New Cytogenetic Approach for G-Banding Analysis}

F. Morato de Oliveira, A.P.N.R. Alves, E.M. Rego, R.P. Falcão

Faculty of Medicine of Ribeirão Preto, University of São Paulo, Ribeirão Preto, Brazil

In this investigation the oligonucleotide DSP30 in combination with IL-2, IL- 6 and IL-10 was used as a B-cell mitogen for cytogenetic analysis in multiple myeloma (MM) and the comparison between the karyotype data obtained by G-banding and FISH profiles from unstimulated cells. Bone marrow mononuclear cells of 55 patients with MM were cultured in RPMI 1640 medium with $20 \%$ fetal calf serum in the presence of the immunostimulatory CpG-oligonucleotide DSP30 and IL-2/IL-6/IL-10 for $72 \mathrm{~h}$. Additionally, 2 extra sets of cell culture were performed for each patient without any stimulant agent (G-banding analysis, when possible, and FISH). The FISH panel included probes for the detection of translocations involving the IGH gene (14q32), gains associated with 11q23-q25 and 1q21 and deletion of 13q14. In the cells treated with stimulus, cytogenetic analysis was possible in 42 patients $(76.5 \%)$, of which $16(38 \%)$ showed a normal karyotype and $26(62 \%)$ an abnormal karyotype. On the other hand, in the group without any stimulus, cytogenetic analysis was successful in 20 patients (36.4\%), i.e., 8 (40\%) with a normal karyotype and $12(60 \%)$ with chromosomal abnormalities. Among the cytogenetic profiles obtained in both groups aneuploidies involving gain of chromosomes $3,5,9,15,16,19$, and 20 , and loss of chromosomes 13,14, 18, and 22 as well as structural abnormalities such as add(1)(p21), inv(3)(q21q26), del(13)(q14), add(14)(q32) and $\mathrm{t}(14$; 16)(q32;q23) were observed. FISH analysis performed in those patients whose bone marrow cells were not stimulated displayed the same chromosomal abnormalities as identified in the group with stimulus. These results indicate that the addition of the immunostimulatory oligonucleotide DSP30 in combination with IL-2, IL-6 and IL-10 effectively induces cell cycle progression of MM cells in vitro.

Financial support: FAPESP (Proc. 07/52462-7 and 11/01647-2).

\section{9 \\ Separation of Myeloid and T-Cells prior to FISH Analysis in Blood Samples from Patients Undergoing Hematopoietic Stem Cell Transplantation}

\section{H. Aviv, S. Payne-Blackman, I. Maxwell, M. Osman}

Robert Wood Johnson Medical School and University Hospital, New Brunswick, N.J., USA

Chimerism assessment after allogeneic hematopoietic stem cell transplantation (HSCT) is used for prediction of graft rejection and disease relapse and is commonly performed by FISH analysis in cytogenetic laboratories in cases of mismatched sex donors. One of the main complications of HSCT is acute or chronic graft versus host disease (GvHD), especially in recipients of in- completely HLA matched donor grafts. Analysis of lineage specific chimerism has recently been identified as a useful biological marker for increased risk of developing GvHD.

Lineage-specific chimerism studies are performed on selected cell fractions, where myeloid chimerism is related to graft rejection or disease relapse, while T-cell chimerism has an impact on GvHD. The cell separation can be accomplished by flow cytometry or by positive selection using magnetic separation.

Following our success with positive selection of plasma cells prior to FISH analysis for plasma cell myeloma, we examined the feasibility of performing cell separation into myeloid and T-cell fractions in our laboratory. We obtained EasySep positive selection human whole blood CD33 and CD3 selection kits from Stem Cell Technologies and evaluated the purity of the separated fractions by flow cytometry. While evaluation of CD3+ cells was straight forward, evaluation of CD33+ cells required anti-CD14/ CD66b antibodies due to blocking of some of the anti-CD33 receptor sites by the positive selection clones.

In a pilot study for validation of our cell separation techniques, we obtained between $99.1 \%$ and $100 \%$ purity in the separated $\mathrm{CD} 3+$ cells and between $90.6 \%$ and $97.1 \%$ purity in the CD33+ cells. There were no CD33+ cells in the CD3+ subset, and no CD3+ cells in the CD33+ subset. We therefore feel that it is feasible to continue with our validation studies and perform cell separation on blood samples of transplant patients in our laboratory.

\section{0 \\ A Comparison of FISH and Microarray Detection of Mosaicism in ALL, CLL, and MDS}

\author{
M.C. Sederberg, G. Gu, C.N. Paxton, L.R. Rowe, K.B. Geiersbach, \\ S.T. South
}

ARUP Laboratories, Department of Pathology, University of Utah, Salt Lake City, Utah, USA

Developments in microarray technology now allow for detection of distinct acquired abnormalities within the cancer genome with kilobase accuracy, something that neither karyotyping nor FISH provides. However, microarray accuracy for reporting mosaicism levels of these abnormalities requires specific evaluation and validation.

To increase our understanding of the reproducibility, accuracy, and detection threshold of likely mosaic cancer-related abnormalities using Affymetrix Cytoscan HD, we selected 30 bone marrow or leukemic blood samples with a diagnosis of ALL, MDS or CLL and FISH-identified abnormalities. Microarray analysis was performed using DNA extracted from uncultured samples. FISH was performed on direct harvests or overnight cultures. Chromosome Analysis Suite (ChAS) software was used to generate the smooth signal for an estimation of percent mosaicism by microarray.

Within the 30 specimens, 60 relevant abnormalities were detected by FISH. Of these 60 abnormalities, all but one was also detected by microarray. The single abnormality not detected was a $13 \mathrm{q}$ deletion present at $5 \%$ by FISH. The lowest levels of detected mosaicism were $a+12$ at $12.5 \%$ and +22 at $18.5 \%$. The smallest

Preprint Cytogenet Genome Res 136/4/2012 
abnormality detected by FISH and microarray was $288 \mathrm{~kb}$. The average deviation between percent mosaicism by FISH and microarray was $12.2 \%$. The degree of deviation between FISH and microarray did not correlate with percent mosaicism, but did correlate with size of abnormality. Smaller abnormalities showed a larger discrepancy of percent mosaicism than larger ones. Culture data were available for 29 FISH abnormalities from direct preparations and for 11 from overnight cultures. The average deviation of mosaicism level for direct preparation was $12.26 \%$ and for overnight cultures was $14.7 \%$.

These findings suggest the smooth signal can provide a reasonable estimation of mosaicism, particularly for levels greater than $15 \%$, and a greater number of probes within a region appear to improve the smooth signal mosaicism estimation.

\section{1 \\ The Carolinas Array Group Database (CAGdb): A Platform-Independent Database of Microarray Findings Shared across Multiple Institutions to Aid in Laboratory Data Management, CNV Interpretation and Submission to ISCA/NCBI ClinVar Datasets}

H.M. Kearney ${ }^{\text {a }}$, W.P. Allen ${ }^{\mathrm{a}}$, A. Chaubey ${ }^{\mathrm{b}}$, B.R.DuPont ${ }^{\mathrm{b}}$, K.A. Kaiser-Rogers ' ${ }^{\text {, C.W. Rehder }}{ }^{\text {d }}$, S.T. South ${ }^{\mathrm{e}}$, J. Tepperberg ${ }^{\mathrm{f}}$, D.J. Wolff ${ }^{\mathrm{g}}$, J. Paschall ${ }^{\mathrm{h}}$, S. Lassiter ${ }^{\mathrm{i}}$, T.J. Hill ${ }^{\mathrm{i}}$

${ }^{a}$ Fullerton Genetics Center, Mission Health System, Asheville, N.C., ${ }^{b}$ Greenwood Genetic Center, Greenwood, S.C., 'Department of Pathology and Laboratory Medicine, Pediatrics and Genetics, University of North Carolina at Chapel Hill, Chapel Hill, N.C., dDuke University, Durham, N.C., ${ }^{\mathrm{e}}$ ARUP Laboratories, Department of Pediatrics and Pathology, University of Utah, Salt Lake City, Utah, ${ }^{\mathrm{f} C y t o g e n e t i c s ~ L a b o r a t o r y, ~ C e n t e r ~ f o r ~ M o l e c u l a r ~}$ Biology and Pathology, LabCorp, Research Triangle Park, N.C., ${ }^{9}$ Medical University of South Carolina, Charleston, S.C., hNational Center for Biotechnology Information (NCBI), NLM, NIH, Bethesda, Md., 'Ninjanetix, Raleigh, N.C., USA

The Carolinas Array Group (CAG) began as an informal network of colleagues in North and South Carolina formed to exchange information between laboratories regarding constitutional microarray interpretations, especially for difficult or rare cases. We have now created a web-accessible database to house each laboratory's abnormal cases that can be freely shared in a de-identified fashion with all participating laboratories. CNV details, interpretation comments, parental and other follow-up data, and clinical features are collected. In addition to manual CNV entry, automated upload of CNV calls exported from array software in.txt file format is supported. The entire database or any subset of search returns can be exported in BED file format or viewed immediately in the UCSC genome browser. Additionally, participating labs are encouraged to submit data to NCBI's ClinVar project through the ISCA data collection. Once entered in the CAGdb site, the automated database program can re-format and submit all selected cases on that laboratory's behalf with no laboratory effort required to interface with NCBI. The program will also sub- sequently re-submit any updated or edited cases. This database is meant to serve as a useful tool, particularly for smaller laboratories, to manage microarray cases, network with colleagues, and to share experience with rare findings. Additionally, this resource is meant to facilitate and encourage submission to large-scale data collections in order to aid in syndrome discovery efforts. We encourage participation from any interested laboratories and invite suggestions for improvement to the functionality of the project. This is operated as a non-profit resource and is freely available to all participants. The database can be accessed at www.carolinasarraygroup.org.

\section{2 \\ Insights from Prenatal Array CGH Analysis: Impact of Cell Culture on Results}

\author{
J.M. Meck, L. Matyakhina, L. Schmidt, V. Nelson, S. Warren, \\ K. Redford, G. Richard, S. Aradhya \\ GeneDx, Gaithersburg, Md., USA
}

Prenatal diagnosis is routinely performed using cytogenetic techniques, such as FISH and chromosome analysis and, most recently, array CGH. We report on select cases that proved challenging due to discrepancies between cultured and uncultured specimens or between different cultures. In our first case, aneuploidy FISH and targeted array CGH were normal on an amniotic fluid specimen from a 32-year-old woman with an ultrasound finding of fetal agenesis of the corpus callosum. However, chromosome analysis revealed mosaic trisomy 8 in 13\% of cells. Subsequent interphase FISH showed trisomy 8 in $39 \%$ of cultured cells, a level of chromosomal mosaicism usually detectable by array. Notably, the array had been performed on uncultured amniotic fluid, suggesting that the trisomic cells had a proliferative advantage in culture. Similarly, cultured and uncultured cells gave different results in the second case. CVS obtained from a 29-year-old woman with a fetal cystic hygroma and an abnormal maternal serum screen showed a normal chromosome result. However, there was a discrepancy between array results on cultured versus uncultured mesenchymal core cells. Cultured cells showed a non-mosaic de novo 3.8-Mb deletion in 16p13.2-p13.3 involving 11 genes, some associated with neurodevelopmental disorders. This deletion was confirmed by interphase FISH. Array results on uncultured mesenchymal core cells suggested a lowlevel mosaic deletion in this region. This case is also consistent with a proliferative advantage of the abnormal cell line in culture. In the third case, low-grade mosaicism for loss of the Y chromosome was detected by array in only 1 of 2 amniotic fluid cultures, demonstrating the threshold of detection of mosaicism for array $\mathrm{CGH}$. These cases raise caution in interpreting prenatal array data from one specimen type and suggest that both uncultured and cultured specimens, or more than 1 culture should be evaluated. 


\section{3}

\section{Towards a Universal Database of Human Genomic Variation: Lessons Learned from the ISCA Consortium}

E.R. Riggs ${ }^{\text {a }}$, E.B. Kaminsky ${ }^{\text {a }}$, V. Patel ${ }^{\mathrm{a}}$, W.A. Faucett ${ }^{\mathrm{b}}$, E. Thorland ${ }^{\mathrm{c}}$, D. Church ${ }^{d}$, J. Paschall ${ }^{\text {, }}$ L. Jackson ${ }^{\text {e }}$, S. Aradhya ${ }^{f}$, R. Kuhn ${ }^{9}$, R. Nussbaum ${ }^{\text {h }}$, H. Rehm ${ }^{i}$, R. Wapner ${ }^{\mathrm{j}}$, D.H. Ledbetter ${ }^{\mathrm{b}}$, C.L. Martin ${ }^{\mathrm{a}}$

${ }^{a}$ Department of Human Genetics, Emory University School of Medicine, Atlanta, Ga., ${ }^{b}$ Geisinger Health Systems, Danville, Pa., 'Department of Laboratory Medicine and Pathology, Mayo Clinic, Rochester, Minn., dNational Center for Biotechnology Information, National Library of Medicine, National Institutes of Health, Bethesda, Md., e'Department of Obstetrics and Gynecology, Drexel University College of Medicine, and Division of Genetics, Children's Hospital of Philadelphia, Philadelphia, Pa., ${ }^{\mathrm{f} G e n e D x}$, Gaithersburg, Md., ${ }^{9}$ Center for Biomolecular Science and Engineering, University of California, Santa Cruz, Calif., ' Division of Medical Genetics, Department of Medicine, University of California, San Francisco, Calif., 'Laboratory for Molecular Medicine, Partners HealthCare Center for Personalized Genetic Medicine, Cambridge, Mass., ${ }^{\text {jDepartment }}$ of Obstetrics and Gynecology, Columbia University Medical Center, New York, N.Y., USA

Genomic variation is inherent amongst all individuals and plays a role in both normal variation and disease. Due to recent technological advances, the ability to detect genome-wide variation is getting easier, while the ability to interpret genome-wide variation remains a significant challenge. Our capability to predict which variants will have biological or health significance versus those that are normal variants is limited. A universal database integrating genotype and phenotype information from thousands of clinical samples within a system of expert-level curation and community engagement would be ideal to address this critical need. Efforts are underway to develop a clinical-grade resource for all types of genomic variation (structural and sequence), modeled on the lessons learned and successes achieved by the International Standards for Cytogenomic Arrays (ISCA) Consortium. Since its inception in 2008, the ISCA Consortium has developed key resources for the implementation and interpretation of chromosomal microarray (CMA) testing, including: a publicly available database of CMA results from clinical laboratories around the world, now including over 30,000 cases; a searchable, web-based interface for this database, allowing users to search by genotype or phenotype; a standard array design, being reevaluated for clinical utility in an evidence-based manner, and a dosage sensitivity map of the human genome. We will present the current efforts of the ISCA Consortium, and how the data collected and tools developed within this project can benefit the cytogenetics community as a whole. We will also discuss plans to align our efforts with those of the sequencing community, to create a universal database of human genomic variation.

\section{4}

Somatic Mosaicism Detected by Exon-Targeted, High-Resolution aCGH in 10,362 Consecutive Cases

J. Pham ${ }^{\text {a }, ~ C . ~ S h a w ~}{ }^{\text {a }, \text { P. Hixson }}{ }^{\text {a }, ~ A . ~ E s t e r ~}{ }^{\text {a }}$, A. Pursley ${ }^{\text {a }}$

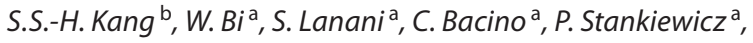
A. Patel ${ }^{\text {a }}$ S.W. Cheung ${ }^{\text {a }}$

${ }^{a}$ Medical Genetics Laboratories, Department of Molecular and Human Genetics, Baylor College of Medicine, Houston, Tex.,

${ }^{b}$ Allina Medical Laboratories, Minneapolis, Minn., USA

Somatic chromosomal mosaicism arising from post-zygotic errors is known to cause several well-defined genetic syndromes as well as contribute to phenotypic variation in diseases. However, somatic mosaicism is usually under-diagnosed due to various factors which include subtle phenotypic abnormalities, technical limitations and inherent tissue-specific mosaicism. Recently, we demonstrated the utility of a custom-designed, exon-targeted whole genome oligonucleotide array to detect intragenic copynumber changes (CNC) in patients with various clinical phenotypes (PMID 20848651). 10,362 patients have been evaluated by this array with indications provided for 8,898 cases. 5,138 patients studied have a clinical indication of cognitive impairment (primarily developmental delay, mental retardation and autism). Somatic mosaicism was detected in a total of 59 cases, which were characterized and confirmed by FISH analysis, chromosome analysis and/or other molecular methods. The level of mosaicism ranges from $6.5 \%$ to $92 \%$. The following abnormal cell lines were detected : (1) aneuploidy including sex chromosome abnormalities and isochromosomes (22 cases); (2) ring or marker chromosomes (13 cases); (3) simple terminal or interstitial deletions/duplications (11 cases); (4) complex structural abnormalities involving 2 or more copy-number changes (5 cases); and (5) derivative chromosomes (3 cases). In addition, mosaic copy-number losses encompassing or disrupting a single gene were detected in 5 cases. Examples of mosaic exonic CNCs occurring within a gene known to be causative for the observed clinical phenotype include: $C A S K$ and FG syndrome 4; IL1RAPL1 and X-linked mental retardation; and TCF4 and Pitt-Hopkins syndrome. In summary, our approach further expands the diagnostic capability of high-resolution array CGH in detecting mosaicism for cytogenetic abnormalities as well as small CNCs in disease-causing genes for patients with autism and intellectual disability.

\section{5 \\ Embryo and Fetal Diagnostics: The Power of SNP Microarrays \\ B. Levy \\ Center for Human Genetics, Columbia University Medical Center, New York, N.Y., USA}

Cytogenetic studies have had a major impact on reproductive medicine, yielding a greater understanding of the frequency of chromosomal abnormalities that occur during gametogenesis, embryonic development and pregnancy. Chromosome abnormalities account for approximately $65-70 \%$ of first trimester mis- 
carriages with aneuploidy accounting for the majority of the cases. Aneuploidy has been found to be relatively common during fetal life, prompting the need for prenatal testing which has now been available for over 40 years. Traditional cytogenetic analysis has been the gold standard for assessing fetal karyotypes and has an accuracy of greater than $99 \%$ for detecting microscopically visible chromosomal abnormalities such as aneuploidy and structural abnormalities. With the recommendation in 2010 by the ACMG to replace karyotyping with chromosomal microarrays as the 'First-Line' postnatal test, attention has shifted toward assessing the utility of microarrays in a prenatal setting. The effectiveness of microarrays has also been assessed in the preimplantation stage where aneuploidy rates are believed to be even higher than at the fetal stage of development. FISH has been used to screen for aneuploidy in human embryos for over 2 decades. Its introduction into the clinical arena offered great potential for enhancing embryo selection. Unfortunately, clinical results have not demonstrated the theoretical improvements in delivery rates of PGD patients which should accompany elimination of chromosomally abnormal embryos from the transfer 'pool'. The blend of wholegenome analysis technologies has led to the development of new assays for comprehensive 24-chromosome aneuploidy analysis. Recent studies using the newer technologies have demonstrated significant improvements in reproductive outcomes and have actually indicated that FISH-based PGD for aneuploidy analysis is less accurate than previously believed. This talk will present current experience with SNP microarrays in both the preimplantation and fetal stages of development.

\section{6 \\ Detection of Masked Hypodiploidy in Pediatric B-Cell Precursor Acute Lymphoblastic Leukemia by Single Nucleotide Polymorphism Arrays \\ X.-Y. Lu ${ }^{\mathrm{a}}$, A. Patel ${ }^{\mathrm{b}}$, J. Ringrose ${ }^{\mathrm{a}}$, Y.-J. Zhao ${ }^{\mathrm{a}}$, P. Rao ${ }^{\mathrm{a}}$, S.E. Plon ${ }^{\mathrm{a}, \mathrm{b}}$, K.R. Rabin ${ }^{\mathrm{a}}$ \\ TTexas Children's Cancer Center, Department of Pediatrics, ${ }^{b}$ Medical Genetics Laboratory, Department of Molecular and Human Genetics, Baylor College of Medicine, Houston, Tex., USA}

DNA ploidy is widely used as a prognostic factor in pediatric B-cell precursor acute lymphoblastic leukemia (BCP-ALL). High hyperdiploidy, defined as chromosome count $\geq 50$, or DNA index (DI) between 1.16-1.60, accounts for $\sim 30 \%$ of the BCP-ALL cases and is associated with more favorable outcomes; whereas, hypodiploidy, defined as chromosome count $<44$, or DI between $0.55-$ 0.81 , accounts for $\sim 2 \%$ of BCP-ALL cases and is associated with a very poor prognosis. Based on chromosome count, 3 hypodiploid subgroups have been described: (1) near-haploidy (24-31 count), (2) low hypodiploidy (32-39 count) and (3) high hypodiploidy (40-43 count). Mosaicism with an exact duplication of the leukemic hypodiploid clone in groups 1 and 2 is also frequently observed. Recently, Carroll et al. [2009] reported a new subgroup - the 'masked hypodiploidy,' which demonstrates hyperdiploidy resulting from a near-duplication of subgroup 1 or 2 , with a unique karyotypic feature consisting of trisomy and tetra- somy involving multiple chromosomes. DNA index and loss of heterozygosity $(\mathrm{LOH})$ have been utilized to detect such 'masked hypodiploidy'. Here, we report detection of masked hypodiploidy using single nucleotide polymorphism (SNP) microarrays. Three cases were tested using SNP array in which 2 of them had been diagnosed as apparent hyperdiploidy but suspected to have a doubled/masked hypodiploidy by FISH/chromosome or DI analyses. SNP array detected the hypodiploid clone in 1 case and revealed a genome-wide $\mathrm{LOH}$ in the second case, showing direct evidence of the doubling of the hypodiploidy. In the third case, SNP array showed mosaicism of the hypodiploid clone and the doubled clone that was consistent with chromosome/FISH results. In conclusion, SNP arrays provide a novel, clinically useful method for detection of masked hypodiploidy by integrating DNA copy number and B-allele frequency in BCP-ALL.

\section{7 \\ An Increased Frequency of Micronuclei in Lymphocytes Is Associated with a Diagnosis of Breast Cancer and the Acquisition/Persistence of a Subset of Chemotherapy-Related Psychoneurologic Symptoms}

N. Aboalela a , D. Lyon ${ }^{\text {b }, ~ R . ~ E l s w i c k ~}{ }^{\text {b , Y.-J. Chen }}{ }^{\text {c }, ~ C . ~ J a c k s o n-C o o k ~}{ }^{\mathrm{a}, \mathrm{c}}$

aDepartment of Human and Molecular Genetics, bSchool of Nursing, 'Department of Pathology, Virginia Commonwealth University, Richmond, Va., USA

Improved survival for women with breast cancer (BC) has been accompanied by the development/persistence of therapy-related psychoneurological symptoms (PNS) that compromise their quality of life. The biological basis for these PNS is unknown, but could reflect the acquisition of chemotherapy-related epigenetic/ chromosomal changes. To test this hypothesis, we initiated a 2 -year longitudinal study ( 5 time points). To date, we have studied 25 women (ages 29-67) with early-stage (stage I, $\mathrm{n}=5$; IIA, $\mathrm{n}=10$; IIB, $\mathrm{n}=9$; IIIA, $\mathrm{n}=1$ ) $\mathrm{BC}$ and have determined lymphocyte micronuclei frequencies (MNF) before, mid-cycle, and 6 months following chemotherapy treatment. The mean MNF in women with $\mathrm{BC}$ prior to treatment $(5 \% \pm 1.6 \%)$ was significantly higher than the mean MNF in age-matched female controls $(1.6 \% \pm 0.87 \%)$ $(p<0.0001)$. Moreover, in the women treated for BC, a significant increase was observed for mean mid-chemo $\operatorname{MNF}(6.9 \% \pm 2.27 \%)$ compared to pre-chemo values $(\mathrm{p}=0.001)$. To date, $11 / 25$ women have been assessed 6 months following chemotherapy and had mean MNF $(6.85 \% \pm 1.86 \%)$ significantly higher than baseline values $(\mathrm{p}=0.009)$. The presence of 2 PNS (depression, anxiety) was analyzed. The MNF was positively correlated with anxiety persisting at 6 months $(p=0.05)$, but not depression $(p=0.83)$. No significant correlations were detected between MNF and age, race, weight, education, or income, but higher MNF were observed at mid-chemo in women with HER2-tumors $(\mathrm{p}=0.009)$ and women receiving doxorubicin/cyclophosphamide versus docetaxel/cyclophosphamide treatment $(\mathrm{p}=0.0005)$. In summary, BC, and/or chemotherapy for BC, is associated with chromosomal instability in lymphocytes that can persist at least 6 months following treatment, and preliminary results show MNF are cor- 
related with anxiety. Further results from our studies may provide insight regarding the persistence of PNS/side effects from chemotherapy in women treated for BC and could possibly lead to the development of tests to identify women most at risk for acquiring PNS.

\section{8}

Genomic Variability by Whole-Genome SNP Genotyping Predicts Time-to-Event Outcome in Previously Untreated Chronic Lymphocytic Leukemia (CLL) Patients

C.D. Schweighofer ${ }^{\text {a }}$, K.R. Coombes ${ }^{\text {b }}$, T. Majewski ${ }^{c}$, L.L. Barron ${ }^{\text {a }}$, S. Lerner ${ }^{\text {d }}$, W.G. Wierda ${ }^{\text {d }}$, A. Ferrajoli ${ }^{\text {d }}$, S. O'Brien ${ }^{\text {d }}$, L.J. Medeiros ${ }^{\text {a }}$, B.A. Czerniak' ${ }^{\text {, }}$ M.J. Keating ${ }^{\text {C }}$, L.V. Abruzzo ${ }^{\text {a }}$

Departments of a Hematopathology, ${ }^{\mathrm{b}}$ Bioinformatics and Computational Biology, 'Pathology, and ' Leukemia, The University of Texas M.D. Anderson Cancer Center, Houston, Tex., USA

CLL is the most common form of adult leukemia in Western countries. Recurrent genomic abnormalities (del6q21, del11q22, del13q14, del17p13, +12 ) are identified by FISH in $60-80 \%$ of patients. del11q22 (ATM, 10-20\% of cases) and del17p13 (TP53, $3-5 \%$ of cases) are among the strongest independent predictors of poor prognosis. We hypothesized that unknown regions of copy number variation $(\mathrm{CNV})$ that affect clinical outcome remain undetected by conventional cytogenetics and FISH, but can be identified by whole-genome SNP genotyping.

We collected blood and clinical data from 168 untreated CLL patients (2000-2008) and compared their SNP genotypes to 73 Caucasian HapMap samples (Illumina Infinium high-density DNA Analysis BeadChips). We identified 322 chromosomal segments of recurrent CNV; 82 occurred more frequently in CLL patients than in HapMap controls ('CLL-specific'). These segments included known recurrent regions of CNV in CLL, and also new loci of acquired or polymorphic CNV. The median number of CLL-specific CNV per patient was 10 (range, 3-21).

In a multivariate Cox proportional hazard model applying the Akaike information criterion, we identified established clinical and laboratory parameters that predicted time-to-treatment. The $I G H V$ somatic mutation status, surface immunoglobulin light chain, and del11q22 or del17p13 independently predicted time-totreatment. We assessed whether any new CNV segments added prognostic value. In a univariate setting, 26 of all recurrent and 13 CLL-specific CNV were associated with time-to-treatment. By multivariate analysis, 14 of all recurrent and 3 CLL-specific CNV segments added independent prognostic value. Genotypic complexity (the number of CNV segments) predicted time-to-treatment independently of established clinical factors.

By comparing SNP genotypes of CLL patients to HapMap controls, we identified new recurrent regions of $\mathrm{CNV}$ associated with time-to-treatment. We established a model to identify genotypic complexity based on any recurrent $\mathrm{CNV}$. We are performing studies to validate our CNV findings, and elucidate their clinical and biological consequences.

\section{9}

Combination of Fluorescence in situ Hybridization (FISH) and Cytogenetic Techniques Optimizes the Diagnostic Process of Patients with Myelodysplastic Syndrome (MDS)

L. Trakhtenbrot, D. Merkel, I. Novikov, A. Nagler, N. Amariglio

Division of Hematology and Laboratory of Hematology, The Chaim Sheba Medical Center, Tel-Hashomer, and Sackler School of Medicine, Tel Aviv University, Tel Aviv, Israel

The myelodysplastic syndromes (MDS) are a group of hematologic disorders characterized by various clonal chromosomal abnormalities (CCA) and a high risk of transformation into acute myeloid leukemia. Cytogenetic analysis is the 'gold standard' for identification of CCA, but it is time consuming while results are often needed within several days. It is considered that in order to identify CCA with $95 \%$ confidence at least 20 metaphases must be analyzed to detect a clone that accounts for $14 \%$ and 30 metaphases to detect a clone that accounts for $10 \%$ of cells.

The aim of our study is to determine the minimal amount of mitoses that must be analyzed by cytogenetic methods to avoid false-negative findings. Cytogenetic and FISH analyses for the most common MDS abnormalities $(-5 / 5 q-;-7 / 7 q ;+8 ; 20 q-$ and $-\mathrm{Y}$ ) were performed on $230 \mathrm{MDS}$ patients. We suggested that determination of CCA minimal frequency by combination of FISH and G-banding may permit reducing the number of mitoses to be analyzed. In 161 cases (92.5\%) with normal FISH, G-banding was also normal, and in 13 cases (7.5\%) 1-2 CCA in minimum $30 \%$ of mitoses (range $30-100 \%$, median $72 \%$ ) were found. In 35 cases with abnormal FISH, G-banding found the same abnormalities as FISH, and in 20 cases complex karyotypes were detected also in minimum $30 \%$ of mitoses (range $30-100 \%$, median $76 \%$ ). Only 1 patient with a complex karyotype had aberrations different from FISH markers in $100 \%$ of mitoses. We could calculate the necessary number of the tested mitoses applying the formula of the total probability and using $30 \%$ probability, because CCA were found with a frequency of at least $30 \%$. Thus, for getting the probability of false negative results less than 0.05 , at least 14 mitoses must be analyzed to detect structural abnormalities or extra chromosomes and 18 for missing chromosomes.

\section{0 \\ Conventional Cytogenetic Analysis and FISH in Myelodysplastic Syndrome (MDS): Are Both Necessary?}

A.E. Wiktor, D.L. Van Dyke, R.P. Ketterling, C.A. Hanson

Department of Laboratory Medicine and Pathology, Mayo

Clinic, Rochester, Minn., USA

The current standard in evaluating suspected MDS includes bone marrow (BM) morphology, conventional cytogenetic analyses (CGA) and MDS-FISH studies. The aim of this study was to determine if MDS-FISH added information beyond CGA in the diagnosis and prognosis of MDS and in the identification of $\operatorname{del}(5 q)$ for lenalidomide therapy.

Preprint Cytogenet Genome Res 136/4/2012 
We identified 498 samples from Mayo Clinic patients (1/0710/11) with BM morphology, CGA and MDS-FISH results. Evaluations with a minimum of $5 \mathrm{MDS}$-FISH probes [including inv(3) (q21q26.2), -5/5q-, -7/7q-, +8, MLL rearrangement, del(13q), del(20q) and in some $\mathrm{t}(3 ; 21)]$ were included. Of 458 cases with $\geq 20$ metaphases, CGA was abnormal in 123 (27\%), normal in 321 (70\%), revealed non-clonal MDS-associated abnormalities in 12 , and 2 had a non-clonal abnormality not targeted by MDS-FISH. MDS-FISH was abnormal in 11/321 (3.4\%) normal CGA and in $2 / 12$ with a non-clonal MDS-associated abnormality. The 13 normal or non-clonal CGA/abnormal FISH cases included: 7 MDS, 3 AML, 2 MM and 1 normal BM; the anomalies detected included: $-7 / 7 q-(n=4),+3 /+3 q 26(n=6),+8(n=5), 20 q-(n=3), 5 q-$ $(\mathrm{n}=2), 13 \mathrm{q}-(\mathrm{n}=2)$, trisomy 5,11 or $13(\mathrm{n}=3)$. The 10 samples with non-clonal CGA/normal FISH included $3 \mathrm{MDS}$ with +8 , 5qor $+8 / 5 q-$. Of 36 CGA with 1-19 metaphases, 11 (28\%) were abnormal CGA and 4/25 (16\%) normal CGA/abnormal FISH. CGA was unsuccessful in 4 cases; FISH was abnormal in 1 case.

When CGA had $\geq 20$ metaphases, there was excellent correlation with FISH (433/458; 94.5\%). FISH did not identify any MDS patients with $5 \mathrm{q}-$ not seen by CGA. An MDS-FISH panel is useful when $<20$ metaphases are identified, with use of select FISH probes to confirm non-clonal MDS-associated abnormalities, or to monitor disease progression on follow-up samples. Morphologic assessment, CGA, and limited MDS-FISH testing offers the most clinically effective and efficient approach in the diagnosis and prognostic assessment of patients with MDS.

\section{1 \\ Transient Clonal Cytogenetic Abnormalities following Chemotherapy for Acute Leukemia}

F. Racke, N. Heerema

Department of Pathology, The Ohio State University, Columbus, Ohio, USA

Determination of karyotype status is an important part of the evaluation of patients newly diagnosed with acute leukemia. Clonal cytogenetic abnormalities (CCAs) are increasingly important in diagnosis and risk stratification of acute leukemia. In addition, monitoring patients for the presence of CCAs is critical for assessment of disease recurrence and clonal evolution of disease. A clone is typically defined as the identification of at least 2 cells harboring the same chromosomal gain or structural abnormality. Occasionally, transient CCAs are observed during the serial monitoring of disease status. In order to evaluate the significance of these transient CCAs, we interrogated our cytogenetic database of acute leukemia patients at OSUMC to look for transient CCAs and to correlate them with disease status. Thirteen patients were identified (12 AML, $1 \mathrm{ALL}$ ) that were found to have transient CCAs that were unrelated to the diagnostic karyotype. Transient CCAs occurred during or in the immediate period of time after therapy and were typically associated with bone marrow findings indicating no active leukemia. While most (9/13) were identified at a single time point, $4 / 13$ were present at different time points or from separate cultures. Most of the transient CCAs were isolated translocations that are not known recurrent CCAs associated with leukemia. In patients who relapsed, the CCAs at relapse were generally consistent with those at diagnosis and the transient CCAs were not present at relapse. Overall, our findings indicate that transient CCAs occur infrequently in patients following treatment for acute leukemia. They are not associated with disease activity and possibly represent genetic injury to hematopoietic progenitors capable of proliferating to the extent that they may be detected but do not have the capacity for long term engraftment as is seen in therapy-related myeloid disease that develops at later time points following chemotherapy.

\section{2 \\ Constitutional t(16;21)(p13;q22) Identified by FISH and Next-Generation Genome-Wide Long Mate- Pair Sequencing Analysis as a Novel Pathogenomic Mechanism in a Series of Three New RUNX1 Mutated FPD/AML Pedigrees: Implications for Diagnostics}

\author{
A. Buijs ${ }^{\text {a }}$ M. Moot ${ }^{\text {a }}$ S. van der Crabben ${ }^{\text {a }}$, B. van der Zwaag a , \\ E. van Binsbergen ${ }^{\text {a }}$, M.J. van Roosmalen ${ }^{a}$, M. Tavakoli-Yaraki ${ }^{a}$, \\ O. de Weerdt ${ }^{c}$, H.K. Nieuwenhuis ${ }^{\text {b }}$, M. van Gijn ${ }^{\text {a }}$, \\ W.P. Kloosterman ${ }^{\text {a }}$
}

aDepartment of Medical Genetics, 'bepartment of Hematology, University Medical Center Utrecht, Utrecht, 'Department of Internal Medicine, St. Antonius Hospital, Nieuwegein, The Netherlands

Familial platelet disorder with propensity to develop myeloid malignancy (FPD/AML OMIM 601399) is an autosomal disorder characterized by quantitative and qualitative platelet anomalies and predisposition to AML, caused by germ line point mutations, deletions and duplications in 21q22 RUNX1. RUNX1 encodes a transcription factor essential for definitive hematopoiesis. Constitutional deletions spanning RUNX1 have been reported in isolated congenital or syndromic thrombocytopenia, stipulating copy number analysis (CNA) of RUNX1 next to sequencing to exclude FPD/AML. Depending on the type of mutation, RUNX1 abnormalities result in negative interference of RUNX1, or act by a mechanism of haploinsufficiency. Given clinical heterogeneity, vigilance in diagnosing FPD/AML is required, reducing the risk of stem cell transplantation (SCT) with affected siblings. The identification of novel mutations is necessary to define clinical features and could contribute to risk stratification for individual cases.

We reported novel c.508G $>\mathrm{A}$ and c.784C $>\mathrm{T}$ RUNX1c mutations in FPD/AML. In a third case, negative for a mutation by sequencing and microarray analyses, we hypothesized a cryptic balanced RUNX1 rearrangement. A t(16;21)(p13;q22) affecting RUNX1 was identified by FISH in leukemic bone marrow. The subsequent detection of affected RUNX1 in cells originating from 2 distinct embryonic germinal layers demonstrated the constitutional nature of the $\mathrm{t}(16 ; 21)(\mathrm{p} 13 ; \mathrm{q} 22)$. Genome-wide long matepair sequencing, a novel next-generation sequencing technology, located the breakpoint to RUNX1 intron 1, likely interfering with $R U N X 1$ transcriptional regulation. This result indicates haploinsufficiency as a pathogenic mechanism for FPD/AML. Our data 
suggest a de novo translocation in an isolated case, presenting as a congenital mosaicism. This is the first report on a constitutional translocation with an oncogene rearrangement (RUNX1) in a hematological malignancy.

The studies delineate a novel pathogenomic mechanism in FPD/AML and indicate elaborated analyses in the diagnostic workup. We will discuss pathogenomic mechanisms in FPD/ AML and isolated/syndromic congenital thrombocytopenia, and propose a RUNX1 diagnostic work-flow.

\section{3 \\ Comparison of Two Microarray (aCGH) Platforms to Assess Global Genomic Status in B-Chronic Lymphocytic Leukemia (CLL)}

\author{
J.G. Rustin ${ }^{\text {, }}$ D.L. Van Dyke a, S.A.Smoley ${ }^{\text {, E.C. Thorland }}{ }^{\text {a }}$, \\ E. Braggio ${ }^{\mathrm{b}}$, N.E. Kay ${ }^{\mathrm{c}}$ \\ ${ }^{a}$ Department of Laboratory Medicine and Pathology, \\ Cytogenetics Laboratory, 'bivision of Hematology-Oncology, \\ Mayo Clinic, Scottsdale, Ariz., 'Division of Hematology, \\ Mayo Clinic, Rochester, Minn., USA
}

CLL has a complicated, clinically diverse behavior. FISH assesses prognostic markers but doesn't evaluate global genomic status. aCGH paints a more complete genomic picture, including genetic complexity. We compared 2 microarray platforms using DNA from CLL patients with 'stable' (not needing therapy after 5 years from diagnosis) or 'progressive' disease (requiring therapy within 2 years of diagnosis). We obtained leukemic B-cells from cells stored at or near diagnosis from 16 stable and 42 progressive patients. Copy number abnormalities (CNA) were compared between 2 platforms: a custom $2 \times 400 \mathrm{k}$ oligonucleotide aCGH chip (Agilent) and the CCMC-designed 4x180k CGH+SNP chip (Agilent). Copy number variants (CNV) were excluded from further analysis.

Both aCGH platforms yielded data for all 58 patients. Excluding known CNV and immunoglobulin rearrangements, the mean number of CNA using the $2 \times 400$ array was 4.0 in the progressive cohort and 2.4 in the stable cohort. On the $4 \times 180 \mathrm{k}$ array, the mean number of CNA was 3.8 and 2.1 in the progressive and stable cohorts, respectively. One $2 \times 400 \mathrm{k}$ sample and two 4 x180k samples had normal aCGH results. The $2 \mathrm{x} 400 \mathrm{k}$ platform detected $43 \mathrm{CNA}$ that were not detected by the $4 \times 180 \mathrm{k}$, whereas $26 \mathrm{CNA}$ were detected by the $4 \times 180 \mathrm{k}$ only (including 5 copy-neutral loss of heterozygosity (cnLOH) calls $>10 \mathrm{Mb}$ on $13 \mathrm{q}$ and one each on $6 \mathrm{q}, 9 \mathrm{q}$, $11 \mathrm{p}, 17 \mathrm{q}$, and $\mathrm{Xp} / \mathrm{q}$ paracentromeric region).

Both platforms confirmed previous FISH panel results (when FISH results were $>23 \%$ ). While the $2 \times 400 \mathrm{k}$ detected more genetic events than the $4 \mathrm{x} 180 \mathrm{k}$, most of these differences were due to variation in probe density and coverage. Only $7 / 43$ (16\%) of those undetected by the $4 \mathrm{x} 180 \mathrm{k}$ are documented recurrent CLLassociated CNA. The distinct advantage of the $4 \times 180 \mathrm{k}$ chip is SNPs to detect cnLOH. Characterization of changes in both DNA copy number and allelic composition will lead to a better understanding of CLL.

\section{4 \\ Genomic Profiling of Rare Disseminated Tumor Cells from Prostate Cancer}

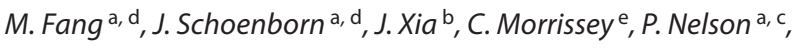
R. Vessella ${ }^{\mathrm{e}}$

${ }^{a}$ Clinical Research Division, ${ }^{b}$ Public Health Sciences, and 'Human Biology Division, Fred Hutchinson Cancer Research Center, ${ }^{d}$ Clinical Cytogenetics Laboratory, Seattle Cancer Care Alliance, 'Department of Urology, University of Washington, Seattle, Wash., USA

Prostate tumor cell dissemination remains a critical challenge in understanding disease progression and effective long-term treatment of patients. Disseminated tumor cells (DTCs) from bone marrow are resistant to chemotherapy and radiation, representing a potential long-lived source for lethal metastases. Very limited knowledge exists about the biology of these rare cells, including the genomic aberrations associated with clinical outcome.

We enriched DTCs from bone marrow aspirates and individually captured them under the microscope. Whole genome amplification (WGA) and high-density SNP-arrays were used to genomically profile rare DTC populations ( $\mathrm{n}=2-25$ cells). We quantified and characterized copy number alterations (CNAs) and loss-of-heterozygosity $(\mathrm{LOH})$ regions in samples isolated from 58 patients taken at the time of radical prostatectomy and from 13 patients with advanced, metastatic or lethal prostate cancer. DTC aberrations were classified by tumor stage and compared to those abnormalities identified in primary and metastatic tumors. Regions of interest were validated with real-time PCR. Extensive optimization of analysis parameters were employed to handle the increased probe variation associated with low input DNA quantity and WGA.

DTCs isolated at the time of radical prostatectomy from earlystage cancer patients are generally heterogeneous and marked with limited focal aberrations, including deletions of less than 100 $\mathrm{kb}$ and, less frequently, gains of less than $500 \mathrm{~kb}$. A considerable number of these CNAs are rare and unique to DTCs. Cancerspecific alterations involve genes of the cadherin family and transcription factors SIX3 and SOX4. Most aberrations are patientspecific and may alter common biological pathways. In contrast, DTCs from patients with advanced-stage prostate cancer typically show frequent and large ( $>1 \mathrm{Mb}$ ) clonal amplifications and deletions, suggesting a high degree of genomic instability common among these patients. Further bioinformatic analysis is underway to gain understanding of the genomic events that drive DTC dissemination, dormancy, and metastatic reactivation in prostate cancer. 
35

\section{Genomic Microarray Analysis Provides an Excellent Genetic Assessment for Oncology: Preliminary Results of the Cancer Cytogenomics Microarray Consortium's Cross-Platform Study}

D.J. Wolffa , J. Biegel ${ }^{b}$, J. Hagenkord ${ }^{c}$, V. Jobanputra d, J. Laffin ${ }^{\mathrm{e}}$, B. Levy ${ }^{d}$, M.M. Li ${ }^{f}$, P.M. Miron 9, F. Monzon ${ }^{\text {h }}$, G. Toruner ${ }^{i}$, M. Rossi ${ }^{j}$, I. Znoyko a , L. Tooke b , D. Wilmoth ${ }^{\text {b }}$, M. Dougherty ${ }^{\text {b }}$, S. Kash ${ }^{\text {c }}$, V. Aggarwal ${ }^{\text {, }}$, O. Nahum ${ }^{d}$, P. Chen ${ }^{f}$, X. Hu ${ }^{f}$, M. Cipollini ${ }^{g}$, K. Alvarez ${ }^{\mathrm{h}}$, D. Streck ${ }^{\mathrm{i}}$

aMedical University of South Carolina, Charleston, S.C., ${ }^{b}$ Children's Hospital Philadelphia, Philadelphia, Pa., ${ }^{~} C$ reighton University, Omaha, Nebr., dColumbia University Medical Center, New York, N.Y., eUniversity of Wisconsin, Madison, Wisc., ${ }^{\text {B Baylor }}$ College of Medicine, Houston, Tex., 9UMass Memorial Medical Center, Worcester, Mass., 'The Methodist Hospital Research Institute, Houston, Tex., 'UMDNJ-New Jersey Medical School, Newark, N.J., 'Emory University, Atlanta, Ga., USA

Cytogenomic microarray analysis provides high-resolution, genome-wide copy number/loss of heterozygosity information that can be used to diagnose cancer, as well as to offer prognostic information and/or to guide treatment. To assess the reliability and intra- and interlaboratory reproducibility of this technology for clinical use, The Cancer Cytogenomic Microarray Consortium (CCMC) conducted a multi-laboratory, multi-platform genomic microarray study utilizing DNA from 29 chronic lymphocytic leukemia (CLL) blood samples, 34 myelodysplastic syndrome (MDS) bone marrow samples and 30 fresh frozen renal cell carcinoma (RCC) samples. Each of the 6 testing laboratories blindly processed the samples according to the clinical standard operating procedures (including laboratory-specific software and calling algorithms) using 1 of 3 microarray platforms: Affymetrix, Agilent, and Illumina, with each platform being used by 2 sites. Raw data from each laboratory was also evaluated independently by a central reviewer who compared results with the known clinical data (FISH for CLL, karyotype for MDS and morphology for RCC). Interpretation of microarray results was concordant with gold standard assays as follows: (a) in CLL, concordant with FISH in 99/104 calls (95\%); (b) for MDS, 91\% concordant with diagnostic cytogenetic findings; and (c) for RCC, concordant with histology in $90 \%$ of cases. Intra- and inter-platform concordance rates were $>90 \%$. Unblinded central review documented that each platform performed equally well and that abnormalities not reported could be detected upon close inspection of the microarray data. Interpretative discrepancies were most often due to results falling below the laboratory's cut-off for reporting. In conclusion, the CCMC study demonstrated: (1) that microarray analysis is comparable or better than current methods used to detect genetic aberrations associated with CLL, MDS and RCC, (2) genetic assessment using microarrays provides reliable and reproducible results, and (3) microarray assessment can be adopted as an effective clinical genetic test for CLL, MDS and RCC.

\section{6}

Insights into the Biology of Acute Leukemia from Genomic Profiling and Next-Generation Sequencing

\section{C.G. Mullighan}

Department of Pathology, St. Jude Children's Research Hospital, Memphis, Tenn., USA

Acute lymphoblastic leukemia (ALL) is the commonest childhood leukemia and represents a range of disorders with distinct genetic alterations that contribute to leukemogenesis. While cytogenetic alterations including aneuploidy and chromosomal alterations are hallmarks of ALL, these are not present in all patients and are insufficient to fully explain the pathogenesis of this disease. Studies performing genome-wide profiling of genetic alterations in ALL using microarray technology and, more recently, next-generation sequencing have transformed our understanding of the biology of ALL, have provided insights into the risk factors driving treatment failure and relapse, and have identified new therapeutic targets that in a remarkably short period of time have entered clinical testing. Microarray analyses have identified over 50 recurring regions of genetic alteration in ALL that are often submicroscopic and often associated with disease subtype. Notably, the transcriptional regulation of B-lymphoid development is perturbed in the majority of B-ALL cases, and specific genetic alterations in this pathway such as $I K Z F 1$ deletions and mutations, are a hallmark of high-risk B-ALL. IKZF1 alterations are common in $B C R-A B L 1$ lymphoid leukemia and in a novel subtype of ' $B C R$ $A B L 1$-like' lymphoid leukemia in which next-generation sequencing has identified a strikingly diverse range of novel rearrangements, sequence mutations and deletions that activate kinase signaling and that are amenable to intervention with currently available kinase inhibitors. Sequencing is also transforming our understanding of T-lineage ALL. Whole genome sequencing of early T-cell progenitor ALL, an aggressive subtype of immature leukemia, has identified a range of mutations targeting hematopoietic development, cytokine receptor and Ras signaling, and chromatin modification in this disease. Together, these findings have provided compelling evidence that in order to 'defeat' ALL we are now obliged to identify all genetic variations in every case, and in order to do so, comprehensive sequencing, coupled with rapid translation to the clinical, is required.

\section{7}

\section{Cytogenetic Biodosimetry: A Nuclear Countermeasures Strategy for the U.S.}

\section{G.K. Livingston ${ }^{\text {a }}$ D.L. Van Dyke}

aRadiation Emergency Assistance Center/Training Site, Oak Ridge Institute for Science and Education, Oak Ridge Associated Universities, Oak Ridge, Tenn., b Department of Laboratory Medicine, Mayo Clinic, Rochester, Minn., USA

Nuclear and radiological terrorism is a threat requiring timely medical interventions to reduce radiation casualties. Timely and proper medical care depends on knowing the victim's radiation dose. Absent physical dosimeters, radiation-specific chro- 
mosome damage studies can serve to estimate the absorbed dose. The dicentric chromosome aberration assay is considered the 'gold standard' to estimate radiation dose because the dicentric frequency correlates positively with the absorbed dose, and it has been validated in numerous radiation accidents over the past 4 decades. Dicentric chromosomes have a low background frequency ( 1 per $10^{3}$ cells) in circulating lymphocytes, are independent of age and gender and relatively easy to identify. Exposure to $100 \mathrm{rad}$ (1 Gy) causes the frequency of dicentrics to increase to about 100 per $10^{3}$ cells and at $500 \mathrm{rad}(5 \mathrm{~Gy})$ the yield is about 1,500 per $10^{3}$ cells. The increase in dicentrics fits a linear quadratic dose response function which can be used as a calibration curve. The assay is labor-intensive; a limited number of cytogeneticists could be overwhelmed by an incident involving hundreds or thousands of persons. The dicentric assay requires cell culture, slide preparation and chromosome analysis. Nuclear preparedness could be strengthened by utilizing laboratory specialists in radiation cytogenetics (few) and clinical cytogeneticists (many) to conduct training, proficiency testing and validation. The ratio of these 2 types of cytogenetic laboratories in the U.S. is about 1 to 70 which demonstrates a large untapped resource of 'chromosome experts' that could support a mass casualty scenario. Our studies confirm that analysis of electronic images results in scoring accuracy and dose estimations equivalent to analysis through the microscope. In conclusion, we believe that cooperation among research and clinical laboratories, along with use of the Internet to score images, would significantly strengthen U.S. radiation emergency preparedness by leveraging clinical and research resources.

\section{8 \\ Alterations in the CDKL5 and MECP2 Genes in Patients with Atypical Phenotypes}

H. Mason-Suares, C.L. Martin, M.K. Rudd

Department of Human Genetics, Emory University, Atlanta, Ga., USA

Rett and Rett-like syndromes are characterized by stereotypic hand movements, regression after the first 6 months of life, seizures, ataxia, acquired microcephaly, breathing abnormalities, intellectual disability, and autistic features. Defects in the methylCpG-binding protein 2 (MECP2) and cyclin-dependent kinaselike 5 (CDKL5) genes cause these syndromes; however, most mutations are single basepair (bp) changes or small deletions $(\sim 20-$ $500 \mathrm{bp}$ ). Larger (>500 bp) MECP2 deletions account for only $\sim 25$ reported Rett cases. Likewise, the majority of CDKL5 mutations are point mutations, although $\sim 10$ deletions/duplications and 2 translocation breakpoints in this gene have been described. Here we report 2 unrelated patients with previously unidentified disruptions of $M E C P 2$ or $C D K L 5$ and atypical phenotypes. The first patient is a 9-year-old female with atypical Rett syndrome and a novel, de novo, $3^{\prime}$ deletion in the MECP2 gene. This patient presented with delayed milestones, speech/language disorder, gross motor delay, significant learning disabilities, unusual impulsive behavior, and hypotonia, but did not have regression, abnormal hand movements or seizures. The deletion is between 4,343 bp and 9,141 bp and was detected on the EmArray 180K microarray.
The minimum deleted region would remove only part of the $3^{\prime}$ UTR, while the maximum could remove part of exon 3 , exon 4 and the $3^{\prime}$ UTR. The second patient is a 3.5-year-old male with a maternally inherited $\sim 160-\mathrm{kb}$ duplication of Xp22.13 that could disrupt the CDKL5, RS1, and PPEF1 genes. This duplication was identified using the EmArray $60 \mathrm{~K}$ microarray. A similar duplication that segregates with retinoschisis and epilepsy has been reported in a Danish family. In contrast, our patient presents with sensorineural hearing loss and hypospadias. Further studies will fine map these alterations to refine the size and breakpoints.

\section{9}

\section{Adult Identical Twins Discordant for a History of Childhood Sexual Abuse Show Higher Frequencies of Acquired Chromosomal Changes in the Abused Twins}

J. Brumelle a, T.P. York ${ }^{\mathrm{b}}$, J. Juusola ${ }^{\mathrm{a}}$, C. Jackson-Cook ${ }^{\mathrm{a}, \mathrm{b}}$

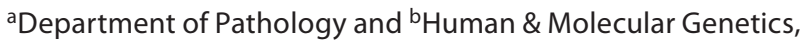
Virginia Commonwealth University, Richmond, Va., USA

Individuals who experienced childhood sexual abuse (CSA) have more health problems in adulthood than individuals who were not exposed to this extreme childhood stress. The biological basis for this association is unknown, but could reflect the acquisition and/or latent manifestation of acquired genomic changes. To test this hypothesis, frequencies of acquired chromosomal changes were determined for adult identical twin pairs discordant $(\mathrm{n}=22)$ and concordant $(\mathrm{n}=2)$ for CSA. Acquired chromosomal changes were assessed by scoring micronuclei (MN) frequencies (cytokinesis-block assay) and chromosome-specific telomere lengths (PNA FISH) in lymphocytes. A significant increase in MN (abnormality) frequencies was observed in the abused twins $(22.03 \pm 11.35)$ compared to their identical unabused co-twins $(14.88 \pm 5.62)(p=0.009)$, but no significant differences were detected between the concordant twins $(p=0.205)$. Interestingly, the divergence in $\mathrm{MN}$ frequencies between discordant co-twins significantly increased with age $(\mathrm{p}=0.0006)$, suggesting a cumulative biological effect. Chromosome-specific telomeric attrition was observed for a subset of telomeres (3p, 5p, 6p, 11q, 13q) in individuals exposed to the most severe form of CSA (intercourse) when compared to their unabused co-twin $(p=0.004)$, but these chromosomes were not clearly over-represented in the MN. Based on spectral karyotyping analyses, chromatin from chromosomes $\mathrm{X}$ and 8 were most frequently observed in the $\mathrm{MN}(\mathrm{p}<0.001)$. In summary, the results of this study, which is the first to evaluate $\mathrm{MN}$ in identical adult twins discordant for CSA, suggests that: (1) increases in acquired chromosomal changes can accumulate in adults who experienced CSA and may mimic advanced aging (consistent with 10 years accelerated aging); and (2) there may be a dose-response relationship between CSA severity and acquired chromosomal changes. Application of the information gained from this study may allow for novel screening techniques to identify individuals at risk for developing stress-associated morbidity in adulthood following childhood abuse. 
40

\section{Microarray Analysis Reveals Mosaicism for Two Abnormal Cell Lines in a Patient with Previously Diagnosed Chromosome 9p Deletion}

D.I. Quigley a , M.I. Dewitt ${ }^{\text {a }, ~ V . J . ~ P a y n e ~ a, ~ O . A . ~ L a m b ~}{ }^{\text {a }}$, C. Miranda ${ }^{\text {b, }}$ D.K. Simpson ${ }^{\text {b }}$

Departments of apathology and ${ }^{b}$ Genetics, Kaiser Permanente NW, Portland, Oreg., USA

We describe an 18-year-old female with severe mental retardation, growth delay and history of a $9 p$ deletion reported during the newborn period. The patient has a history of repaired VSD and PDA, very limited speech, behavioral issues and recent development of seizures. Streaky pigmentation on the abdomen and back were also noted. The patient was referred to genetics for updated evaluation. Chromosome and microarray analyses were requested to confirm 9p deletion and to characterize the deleted region.

Routine chromosome analysis confirmed a terminal 9p23 deletion. Microarray results using the Illumina HumanOmnilQuad array suggested trisomy 9, with a slight quantitative shift but clear SNP allele tracks consistent with 3 copies of chromosome 9. A very small terminal deletion at 9p24 was also evidenced, but there was no evidence for the 9p23 deletion. To clarify these findings, FISH studies were performed, which showed evidence for trisomy 9 and deletion $9 p$ in $28 \%$ and $72 \%$ of interphase cells, respectively. Metaphase FISH analysis showed an apparent isodicentric chromosome 9 which was finally observed cytogenetically by more extensive metaphase search. The isodicentric 9 cell line was observed in 6/50 (12\%) metaphase cells.

This study showed previously undiagnosed mosaicism for an isodicentric chromosome 9 (essentially trisomy 9) in a patient with known deletion 9p23 and severe cognitive deficits. The isodicentric 9 cell line was likely not recognized due to low level mosaicism and relatively poor quality of metaphase cells compared to the deletion 9p23 cells. The likely origin of the chromosome findings was instability of the isodicentric chromosome 9. The deletion 9p23 cell line resulted from isodicentric breakage during cell division. There was no evidence for the reciprocal cell line from the isodicentric break or for a normal cell line in peripheral blood.

\section{1 \\ High Density SNP Microarray Analysis of Products of Conception}

K.B. Geiersbach a, c, C.N.Paxton ${ }^{\text {d }}$, A.N. Lamb ${ }^{\text {a, c }}$, A.R. Brothman ${ }^{\text {a-c, }}$, S.T. South ${ }^{\mathrm{a}-\mathrm{c}}$

Departments of apathology and bediatrics, University of Utah School of Medicine, 'Cytogenetics Laboratory, ARUP Laboratories, ${ }^{\mathrm{d}}$ ARUP Institute for Clinical and Experimental Pathology, Salt Lake City, Utah, USA

Genomic microarray analysis is a new method for whole genome analysis of products of conception (POCs) which can mitigate technical challenges related to cell culture. We have characterized a total of 68 POCs using 2 Affymetrix SNP microarrays
(SNP Array 6.0 and CytoScan). Twelve samples were tested on both platforms. A variety of tissue types (53 villi, 8 fetal somatic tissue, and 7 amniocyte) and preparations (15 fresh uncultured, 21 frozen uncultured, and 32 cultured samples) were tested. Quality of extracted DNA (as measured by 260/280 and 260/230) and array QC metrics did not differ significantly between sample types. Concordance between platforms was $100 \%$ for clinically significant abnormalities, but SNP 6.0 yielded a higher rate of false or ambiguous calls due to wave artifact. Triploidy, but not tetraploidy, was detectable on both platforms using the allele track feature. Six out of 53 samples characterized by cytogenetics showed discrepant findings on microarray; 3 cultured specimens were characterized as normal female on microarray due to maternal cell contamination, and 3 uncultured specimens showed discrepant findings due to mosaicism. Mosaic cases included (1) villi with a 47,XX,+7 karyotype versus normal female array; (2) fetal somatic tissue with a 46,XX,del(13)(q32)[6]/46,XX[16] karyotype versus normal female array; and (3) villi with a 45,XY,-21 karyotype versus gain of chromosome 9 and mosaic loss of chromosome 21 on array. Sample identity was confirmed for all discrepant cases. This series demonstrates that high density SNP microarray is a useful tool for whole genome analysis of POCs, and a variety of sample types can be used; however, the limitations of each sample type must be considered. Current evidence supports use of microarray as an ancillary diagnostic method, particularly for samples that fail to grow in culture, and further studies are needed to determine the abnormality detection rate.

\section{2 \\ SNP POC Microarray Analysis - Primary Cytogenetic Test for POC \\ J. Tepperberg ${ }^{\text {a }, ~ H . ~ T a y l o r ~}{ }^{\text {a }}$, R.D. Burnside ${ }^{\mathrm{a}}$, B. Rush ${ }^{\mathrm{a}}$, I.K. Gadi ${ }^{\text {, }}$ \\ V. Jaswaney ${ }^{\mathrm{a}}$, E. Keitges ${ }^{\mathrm{b}}$, R. Pasion ${ }^{\mathrm{a}}$, K.L. Phillips ${ }^{\mathrm{a}}$, V.R. Potluri ${ }^{\mathrm{c}}$, \\ H. Risheg ${ }^{\text {b }, ~ S . ~ S c h w a r t z ~}{ }^{\text {a }}$, J.L. Smith ${ }^{\text {C }, ~ P . R . ~ P a p e n h a u s e n ~}{ }^{\text {a }}$ \\ a LabCorp, Research Triangle Park, N.C., ' LabCorp, Seattle, Wash., 'LabCorp, Houston, Tex., USA}

Microarray testing of POCs has clearly demonstrated significant diagnostic advantages compared to routine chromosome analysis and FISH. Over the past year, approximately $75.0 \%$ of POCs grew in culture and were analyzable by G-bands while $96.0 \%$ of POCs ordered for the array provided informative results. Approximately $88.0 \%$ of samples that did not proliferate in culture provided a microarray result and of these, $46.6 \%$ were abnormal. The ratio of male to female samples tested by the array was close to $50: 50$ compared to $77.0 \%$ female and $23.0 \%$ male by routine chromosome analysis. Of the abnormal results, both Gbanding and microarray showed approximately $71.0 \%$ were aneuploid. Some of abnormalities for both methods included trisomies $4,6,7,9,10,13,15,16,18,21,22$, monosomy $\mathrm{X}$ and multiple aneusomies. Turner syndrome was the most frequent finding for both methods. Three cases showing normal chromosomes (two $46, \mathrm{XX}$ and one 46,XY) demonstrated extensive homozygosity consistent with a diagnosis of complete moles. Five triploids were identified based on the genotype allele pattern. Dosage, as in any array was normalized to 2 copies and thus would have been missed 
by non-SNP microarray assays. In addition, 9 cases had copy number changes between 1 and $10 \mathrm{Mb}$ that more than likely would have been missed by routine G-banded studies. Thus, the SNP array provides superior performance to cultured chromosome analysis, provides a more accurate percentage of fetal tissue with less MCC compared to chromosome analysis, increases the overall success and detection rate, accurately identifies partial and complete moles, and can effectively be performed on uncultured tissue as well as tissue that failed to grow in culture. The results clearly illustrate the effectiveness of SNP array analysis on POC tissue and demonstrate its utility as a primary cytogenetic test for POC evaluation.

\section{Poster Presentations Constitutional Cytogenetics}

\section{3 \\ Microdeletion of 17q24.3 Detected by Chromosome Microarray Analysis Is Associated with Pierre Robin Sequence due to Position Effect on SOX9}

I.E. Amarillo a , K. Dipple ${ }^{\text {b }}$, F. Quintero-Rivera ${ }^{\text {a }}$

${ }^{a}$ Clinical Cytogenetics, UCLA, ${ }^{b} 695$ Charles E. Young Drive South, Los Angeles, Calif., USA

A Pierre Robin Sequence (PRS) is a complex of abnormalities that occurs during embryological development. It is considered a cranial neurocristopathy, and defined by a core triad - micrognathia, glossoptosis and a posterior U-shaped cleft palate. It occurs in $1 / 2,000-1 / 10,000$ neonates. PRS can be a part of a clinically wellestablished syndrome or an isolated event. The genetic basis of the latter is poorly understood. Heterozygous deletions, translocation breakpoints, point mutations and positional effect of some loci/ genes have been associated with PRS. These include 2q24.1-q33.3 (GAD67), 11q21-q23.1 (PVRL1) and 17q21-q24.3 (SOX9). Here we report a 15-month-old female referred to Genetics because of isolated PRS. SNP chromosome microarray analysis detected a $499 \mathrm{~kb}$ heterozygous copy number loss at 17q24.3 (bp 68755732-69332449 hg19; 499 oligonucleotides); this region is devoid of any RefSeq genes. However, this copy number loss maps $784 \mathrm{~kb}$ upstream of the $5^{\prime}$ of the SOX9 gene. Microdeletions that are up to $1.5 \mathrm{Mb}$ upstream or downstream of SOX9 have been established to have position effect on SOX9, due to elimination of highly-conserved noncoding cis regulatory elements. Moreover, this patient's deletion falls within the specific region on $17 \mathrm{q} 24.3$ recently associated with PRS. This report is the first to provide evidence through the use of chromosome microarray analysis in the clinical setting, for position effect on SOX9 associated with PRS, which is site-specific, and different from that associated with campomelic dysplasia. It also highlights the fact that copy number losses in gene-desert regions can be associated with human congenital malformations and have to be interpreted with caution. Finally, it supports the existence of aberrations of 17q24 locus underlying PRS.

\section{4}

Molecular and Clinical Characterization of Patients with Tetrasomy and Pentasomy 15q11q13

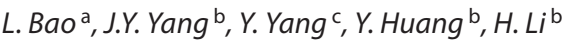 \\ aDivision of Human Genetics, Department of Pediatrics, \\ Cincinnati Children's Hospital Medical Center and College \\ of Medicine, University of Cincinnati, Cincinnati, Ohio, USA; \\ ${ }^{b}$ Center for Clinical Molecular Medicine, Children's Hospital \\ of Chongqing Medical University, Chongqing, 'Shanghai \\ Children's Hospital, Shanghai Jiaotong University Affiliated \\ Children's Hospital, Shanghai, China
}

Chromosome 15q11q13 contains a group of imprinted genes that play an important role in development. Deletions of paternal and maternal 15q11q13 segment have been associated with Prader-Willi syndrome and Angelman syndrome, respectively. Although duplications of 15q11q13, particularly inherited maternally, have been reported in patients with developmental delays and autism spectrum disorders, few reports on tetrasomy and pentasomy for 15q11q13 are documented in the literature, and relationship between 15q11q13 genomic dosage and clinical phenotypes remains to be further defined. Here, we report 2 patients presenting with developmental delay and mental disability. Conventional chromosome banding analysis showed a large derivative chromosome 15 in both patients: 47,XY,+del(15)(q14) in patient 1 and $47, \mathrm{XY},+\operatorname{del}(15)$ (q21q26) in patient 2. Microarray and FISH analysis unveiled that the derivative chromosomes 15 resulted from either duplication or triplication of a $12 \mathrm{Mb}$ genomic $15 \mathrm{q} 11 \mathrm{q} 13$ segment containing the PWS/AS critical region. The derivative chromosome 15 in patient 1 had 2 copies of 15q11q13 and 3 copies of $15 \mathrm{q} 11 \mathrm{q} 13$ in patient 2 . The karyotypes were revised to $47, \mathrm{XY},+\mathrm{iso}(15)(\mathrm{q} 13)$ (tetrasomy for 15q11q13) and 47,XY,+trp(15) (q11q13) (pentasomy for 15q11q13) in patient 1 and patient 2, respectively. Genotyping analysis and methylation analysis demonstrated that both derivative chromosomes 15 were of maternal origin with a maternal methylation pattern. Both patients had clinical features including hypotonia, dysmorphic features and intellectual impairments, but clinical features were more severe in patient 1 with tetrasomy 15q11q13 than in patient 2 with pentasomy 15q11q13, suggesting a dosage-dependent pattern between 15q11q13 segment and clinical phenotype. Our study underscores the importance of array analysis in characterizing chromosomal abnormalities and sheds some light on roles of 15q11q13 aberrations in developmental delay and mental impairment. 


\section{5}

\section{The Next Step in Clinical Cytogenetics: Moving Beyond Empirical Rules}

R.G. Best ${ }^{\mathrm{a}, \mathrm{b}}, \mathrm{G}^{\mathrm{K}}$ Khushf ${ }^{\mathrm{b}}$

aBiomedical Sciences Department, University of South Carolina, School of Medicine, Greenville, S.C., bUniversity of South

Carolina, Center for Bioethics \& Medical Humanities, Columbia, S.C., USA

Clinical cytogenetics is an essentially empirical science, and little is understood about the mechanisms by which constitutional cytogenetic abnormalities disrupt development and function. We have come to recognize a rule set, derived from empirical observation, that operates effectively at the level of banded chromosomes. We realize, for example, that autosomes exhibit chromosome balance, with 2 and only 2 copies of each segment of the genome being the norm; and that monosomy is generally more deleterious than trisomy. However, we have no working theory that explains how or why monosomy or trisomy should be disruptive at all, far less any theory that could predict specific phenotypes that would be associated with gain or loss of any specific segment of the genome. Mendelian theory does not address why an extra copy of a chromosome should be problematic at all, nor does it predict that trisomy 21 should result in the Down syndrome phenotype. Indeed, more than a half century after the discovery that constitutional trisomy 21 uniformly predicts a Down syndrome phenotype, we have only the crudest explanations as to why or how. This is in stark contrast with the molecular genetics of Mendelian traits where we both can predict clinical phenotypes based on laboratory observations, and also account for the pathophysiologic mechanisms that constitute a causal chain connecting specific molecular lesions with their phenotypic manifestations.

We consider 2 fundamental types of uncertainty in clinical cytogenetics that obscure our understanding of how and why chromosome abnormalities disrupt the phenotype: ontic and epistemic. The emergence of array CGH and whole genome sequencing offers the opportunity for disentanglement of these 2 elements of uncertainty, and marks a transition into an era in which we might expect to ultimately discover and understand the causal chain of events underlying chromosomal syndromes.

\section{6 \\ A Comparison of FISH and Microarray Technologies for Detecting Mosaicism for Trisomy 21}

C. Charalsawadi a , P. Papavasiliou ${ }^{\text {a, c }}$, C. Dumur ${ }^{\text {, }}$, A. Ferreira-Gonzalez ${ }^{\text {b }, ~ T . P . ~ Y o r k ~}{ }^{\mathrm{a}}$, C. Jackson-Cook ${ }^{\mathrm{a}, \mathrm{b}}$

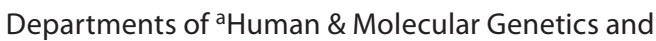

bPathology, Virginia Commonwealth University, Richmond, Va.,

'Department of Pathology, Duke University, Durham, N.C., USA

Microarray technology has resulted in better recognition of small constitutional chromosomal imbalances. However, the utility of this assay for detecting mosaicism is controversial. Therefore, the primary aim of this study was to compare the results of interphase FISH methodology to those from a SNP-based micro- array platform (Affymetrix 6.0) for detecting/quantifying constitutional mosaicism. Study subjects included individuals having Down syndrome with mosaicism $(\mathrm{n}=15)$ and non-mosaic controls having trisomic $(n=6)$ and euploid $(n=17)$ chromosome 21 complements. The DNA samples, which were coded so investigators were unaware of the specimens' karyotype status at the time of analysis, included both archival ( $\mathrm{n}=22 ; 3-20$ years old) and recent cases ( $\mathrm{n}=16 ; 0.5-2$ months old). The number of chromosome 21 FISH signals present was determined by scoring 1000 or 500 nuclei from cultured or uncultured blood samples, respectively, with these values ranging from $8.5-93.6 \%$. A significant positive correlation between the mean smoothed $\log 2$ array ratios and percentage of trisomic cells quantified with FISH was observed $(r=0.86, p<0.01)$. A smooth signal analysis of the array data resulted in consistent classification of all positive and negative controls, with $9 / 15$ mosaic cases being classified as mosaics $(41.8 \pm 15.2 \%$ mean trisomic cells by FISH); $2 / 15$ as full trisomy $(85.8 \pm 7.4 \%$ by FISH); $2 / 15$ as equivocal ( $15.9 \pm 9.0 \%$ by FISH); and $2 / 15$ as normal ( $16.8 \pm 4.8 \%$ by FISH). One patient had the unexpected observation of mosaicism for trisomy 14 (confirmed by FISH). No significant difference was detected for the number of phenotypic traits present in the mosaic probands classified as full trisomy $($ mean $=17$ traits); mosaic $($ mean $=14)$; equivocal (mean $=13)$; or negative $($ mean $=11)$. Overall, these results suggest array-based technology is effective for detecting mosaicism present in $20 \%$ or more of cells, but may be less consistent in detecting low levels of mosaicism ( $<15 \%$ trisomic cells).

\section{7 \\ Abnormal Recombination between Yp11.2 and Xq21.3 Homology Loci as a New Mechanism of X-Y Translocations and Terminal Xq21.3/Xq22 Deletions \\ V.B. Chernykh, L.F. Kurilo, V.A. Galkina, M.E. Karmanov, \\ Y.O. Kozlova, M.E. Minzhenkova, O.P. Ryzhkova, A.L. Chukhrova, A.V. Polyakov \\ Research Centre for Medical Genetics of the Russian Academy of Medical Sciences, Moscow, Russia}

$\mathrm{X} ; \mathrm{Y}$ translocations often result in transfer of a Yp fragment, including the $S R Y$ gene, onto the $\mathrm{X}$ chromosome, the events leading to gonadal development abnormalities. Commonly XX maleness is a result of Yp-Xp recombination between $P R K X / Y$ genes. However, the mechanism of other translocations is still unknown.

To evaluate the mechanism of $\mathrm{X} ; \mathrm{Y}$ translocations, we examined 103 patients with disorders of sexual development (DSD): forty 46,XX males, a 45,X male, fifty 46,XY females with gonadal dysgenesis, a 47,XYY female, 12 individuals with true hermaphroditism, ovotesticular DSD (46,XX, $\mathrm{n}=10$; $\operatorname{mos} 46, \mathrm{XX} / 47, \mathrm{XX}$, +mar, $\mathrm{n}=1$; and 46,X,del(X)(q22), $\mathrm{n}=1)$. Chromosome analysis was performed using G-banding. FISH analysis was done on +metaphase chromosomes and interphase nuclei with DXZ1, DYZ3 and LSI SRY probes. PCR amplification of SRY, AMELX/Y, $Z F X / Y$ and 27 Y-specific loci was performed.

Eighty percent of the 46,XX males, the $45, \mathrm{X}$ male and 2 of 12 patients with 46,XX ovotesticular DSD were $S R Y+$; the 46,X,del(X) (q22)/47,XY,del(X)(q22) mosaic, the 47,XYY female and five 
46,XY patients were $S R Y-$. FISH analysis demonstrated a translocation of a Yp11 fragment on Xp22.3 in (Yp+)XX sex reversed individuals, and on Xq21.3 in the abnormal X chromosome in 1 $S R Y+$ true hermaphrodite with a karyotype of 46,X,del(X)(q22). Molecular analysis demonstrated differences in breakpoints between patient groups, $S R Y-\mathrm{Y}$-derivates and $S R Y+\mathrm{X}$-derivates. The breakpoints in $S R Y-47, X Y Y$ and 46,XY females are in Yp11.31, while in (Yp+)XX sex-reversed individuals 7\% of the breakpoints are in Yp11.31 and 93\% are in Yp11.2. One-third of the translocations show breakpoints in the Yp11.2/Xq21.3 homology region (Y-deletion intervals $1 \mathrm{~B}-3 \mathrm{~A})$. Excluding 1 case, all (Yp+ class III)XX maleness were detected on a Yp pericentric inversion background, due to ectopic Yp-Xp recombination between PRKX/ $P R K Y$ genes, which resulted in 57\% SRY+ 46, XX males.

These results indicate that most Y-derivates resulting from Yp;Xp translocations commonly present more distal Yp breakpoints than X-derivates. We hypothesize that recombination between Yp11.2 and Xq21.3 loci (near or within $P C D H 11 X / Y$ and TGIF2LX/Y genes) is one of the mechanisms of X-Y translocations and $\mathrm{Xq} 21.3 / \mathrm{Xq} 22$ deletions.

\section{8 \\ Double Autosomal Trisomy in a Liveborn Term Fetus: $47, X Y,+9 / 47, X Y,+18$}

P.T. Greipp ${ }^{\text {a }}$ E.B. Mitchell ${ }^{\mathrm{a}}$, M.J. Wick ${ }^{\mathrm{b}}$

aDepartment of Laboratory Medicine and Pathology: Cytogenetics, 'bepartment of Medical Genetics, Mayo Clinic, Rochester, Minn., USA

Double autosomal trisomy (DAT) is an uncommon occurrence in prenatal diagnosis. It is rare in a liveborn fetus, as most DAT conceptuses abort spontaneously.

Herein we report DAT in a liveborn male term infant of a chromosomally normal primiparous mother (32 years) and father (34 years). Pregnancy was complicated by severe early intrauterine growth restriction (IUGR) and a one-vessel cord. Fluorescence in situ hybridization (FISH) on amniotic fluid at $22 \mathrm{w} 6 \mathrm{~d}$ gestation showed a normal signal pattern for chromosomes X, Y, 13 and 21 . However 127/200 nuclei contained 3 signals for chromosome 18. G-banded karyotype on cultured amniocytes revealed $47, \mathrm{XY},+18[21] / 47, \mathrm{XY},+9[4]$. No normal metaphases were observed, suggesting a double meiotic non-disjunction event.

Elective caesarian section was performed at 41 weeks. At birth there was no spontaneous cry, poor respiratory effort and minimal movement. Birth weight $1.7 \mathrm{~kg}$. The neonate was small for gestational age with decreased subcutaneous fat and pale dusky skin. No cephalic deformities and normal fontanelles. Heart tones present without murmur at $50 \mathrm{bpm}$. Capillary refill was prolonged. Abdomen was scaphoid. Male genitalia appeared normal. Mild deformities of extremities suggested oligohydramnios. Phalanges were not overlapped. Comfort measures were employed and the baby expired $4 \mathrm{~h}$ later.

Cord blood karyotype, 47,XY,+18[22]/47,XY,+9[8], confirmed prenatal diagnosis. FISH showed cord nuclei: $+18(66 / 100),+9$ (48/200) and placental nuclei: $+18(62 / 100)$ and $+9(99 / 200)$. Anomalies were in separate cell lines.
The specific cytogenetic abnormalities identified in this patient have not previously been described in an abortus or a liveborn infant. Phenotypic features of IUGR and limb deformities appeared similar to isolated trisomy 18 (T18) and isolated trisomy 9 (T9). However, several features were not present; Pierre Robin sequence and cleft palate (T18) and cerebral/cerebellar malformations (T9). Additional reports of DAT will contribute to our understanding of the associated phenotype and offer opportunities to examine segregation errors in non-disjunction.

\section{9 \\ Comprehensive Assessment and Validation of the Automated Metaphase Finder GSL-120}

J.C. Hodge, C.L. James, D.A. Duncan, R.C. Mulder

Department of Laboratory Medicine and Pathology,

Mayo Clinic, Rochester, Minn., USA

The GSL-120 automates microscope slide scanning and metaphase image capture. Object images are acquired on low magnification $(10 \times)$ according to manufacturer's specifications. Objects are then denoted as likely metaphases of interest for potential high power $(100 \times)$ image capture by applying a 'classifier' created through software training with cases and manual input on proper selection.

To validate the GSL-120 for clinical practice, a prospective study of 101 non-pediatric consecutive bone marrow clinical cases were blinded for concurrent scanning on the GSL-120. Interwoven were de-identified retrospective cases of 53 known abnormals encompassing low level mosaics, low mitotic index, euploidy/aneuploidy, or subtle structural abnormalities. All cases were analyzed for karyotype and image quality.

The GSL-120 generated images of sufficient quality. The blinded and clinical case karyotypes matched at $97.4 \%$ after removal of discrepant results due to independent technical issues. These included insufficient metaphases for analysis in retrospective cases with small pellets (5 cases; $3.2 \%$ ) and single abnormal metaphases in prospective cases that could represent culture artifact ( 4 cases; $2.6 \%$ ). Cases with 2 abnormal metaphases in one analysis but normal results in the other analysis occurred both against and in favor of the GSL-120, which is expected for low level clones and are therefore not considered truly discrepant (3 cases of either trisomy 8,11 , or $15 ; 1.9 \%)$.

The remaining $2.6 \%$ (4 cases) were of concern as reportable near-haploid/near-tetraploid clones occurred in low power but not high power images. The classifier was therefore further trained with such cases and a second validation involving 170 prospective cases was undertaken. All near-haploid/near-tetraploid cases were identified while discordant single abnormal metaphases or low level clones persisted (9 cases; 5.3\%). Overall, the GSL-120 captures quality representative metaphases following careful training of the classifier to recognize a range of total counts and chromosome morphologies. 


\section{0}

\section{Mosaicism of a Normal Female Karyotype and an} Unbalanced Autosomal Reciprocal Translocation

K. Huijsdens-van Amsterdam ${ }^{\text {a }}$, M. van Seggelen ${ }^{\text {a }}$, M.C. van Maarle ${ }^{\text {a }}$, C.J. Bax ${ }^{\text {b }}$, A.C. Knegt ${ }^{\mathrm{a}}$

aDepartment of Clinical Genetics, ${ }^{\text {b Department of }}$

Fetal Medicine, Academic Medical Center, Amsterdam, The Netherlands

We present a prenatal sample displaying mosaicism of an unbalanced autosomal reciprocal translocation and a normal female karyotype. Chorionic villus sampling was performed because of echographic abnormalities (omphalocele). In trophoblast cells, a normal female karyotype was seen. There was insufficient material for a long term culture of mesenchymal cells.

Echographic screening at 20 weeks of pregnancy revealed multiple abnormalities besides the omphalocele, among which were a complex heart defect and microcephaly. Therefore, amniotic fluid was obtained. In all 10 metaphases analyzed, an aberrant karyotype was seen with a derivative chromosome 13. MLPA for subtelomeric rearrangements (P070, MRC Holland) showed a duplication/deletion of the subtelomeric regions of the long arms of chromosome 3 and chromosome 13, respectively. Thus, the derivative chromosome 13 seen is in fact due to an unbalanced translocation between chromosomes 3 and 13: $\operatorname{der}(13) t(3 ; 13)$ (q2?1;q34), mlpa 3qsubtel(P070) ×3,13qsubtel(P070) $\times 1$.

The pregnancy was terminated at 23 weeks and 4 days, and placental tissue was examined. FISH analysis was performed with probes for chromosomes 3 and 13 (probe BCL6 region 3q27, and probe LSI 13 region 13q14, Abbott-Vysis). Short term culture of 5 biopsies taken showed a normal karyotype in 6/7 metaphases analyzed. The majority of the nuclei scored (223/249 nuclei, 90\%) had a normal signal pattern. The remaining 10\% (26/249 nuclei) showed an abnormal signal pattern: 3 signals for region $3 q 27$ and 1 signal for region 13q14. Long term culture of the biopsies showed an aberrant karyotype in the majority of the metaphases $(24 / 25$, $96 \%)$.

Thus, chorionic trophoblast cells mainly contained normal female cells $[10 \%$ der(13)], whereas the mesenchymal cells of the chorionic villi and the amniotic fluid mainly showed a derivative chromosome 13 [96\% der(13)]. Possible mechanisms for the formation of a mosaic pattern of a normal cell line and an unbalanced autosomal translocation are discussed. Karyotyping of parental blood will be performed.
51

Autosomal Structural Mosaicism - A Review of Cases from Eleven Years of Constitutional Karyotyping Experience

\author{
A.M. Joseph-George, D. Antinucci, G. Nie, I. Teshima, \\ D.J. Stavropoulous, M. Shago
}

Cytogenetics Laboratory, Division of Molecular Genetics, Department of Paediatric Laboratory Medicine, The Hospital for Sick Children, Toronto, Ont., Canada

Chromosomal mosaicism is the presence of more than one karyotypically distinct population of cells within a single organism. It arises post-zygotically, is variable in its extent between different tissues and is not always straightforward to ascertain in the laboratory when present at low levels. From a clinical perspective, phenotypic presentation related to the mosaicism may be variable and could be interpreted as normal variation in the population.

A retrospective review of approximately 27,800 cases was undertaken to establish the general prevalence of mosaicism in our patient population and to then examine further a subset of these cases, specifically those with structural mosaicism for an autosome. These patients, the majority pediatric, were referred for varied indications to this diagnostic centre for postnatal constitutional karyotyping of their peripheral blood by G-banding analysis.

Autosomal structural mosaicism is rare and can involve intrachromosomal, interchromosomal or complex rearrangements with alteration resulting in balanced or unbalanced states. A summary of all cases with mosaicism will be presented, including a breakdown of cases within the different categories. Karyotypes for approximately 31 cases, mosaic for a structural rearrangement, will be examined for the chromosome and region involved, breakpoints, type of rearrangement and extent of imbalance (if any) to identify trends or any preferential chromosomal involvement. Results of microarray analysis, where available, will also be included. Cases involving more complex mosaic karyotypes will be described in detail.

\section{2}

\section{The Triumphs and Challenges of Very High Density SNP Microarray Analysis in a Non-Academic Clinical Setting}

J. Kogan, C. Booth, D. Rita, A. Catic

ACL Laboratories, Rosemont, III., USA

The development of high resolution genomic microarray analysis has been proceeding at a dizzying pace. As the density of markers on the arrays continues to increase, the ability to detect smaller and smaller copy number changes increases. What is less clear is whether this progress is paralleled by a similar increase in the ability to give useful information to our patients. Our laboratory has recently switched from the Affymetrix Genome-Wide Human SNP Array 6.0 to the higher resolution genecentric CytoScan HD SNP microarray platform for postnatal 
constitutional studies, which has presented us with both advantages and challenges. Clinically, the benefits of high resolution microarray analysis for certain patients are clearly quite significant, sometimes providing potentially lifesaving information. In other cases, however, the information causes confusion or anxiety in patients and their families. Technically, the challenges of interpretation of microarray results lie in 2 areas. First, confirming very small deletions and duplications is sometimes problematic, particularly for a non-academic laboratory such as ours. Standard FISH probes are often too large to be useful, and molecular methods may not always be available. Second, interpretation of these findings can be challenging when the majority of publicly available data is based on lower resolution arrays, and thus there is a paucity of high resolution data to which to refer. Our laboratory has addressed some of these issues in creative ways, yet some needs are still unfulfilled. Several interesting cases will be presented, demonstrating both the usefulness and the challenges of the current high resolution microarray platforms in the clinical setting.

\section{3 \\ Phylloid Hypomelanosis in a Patient with Mosaic Tetrasomy 13q}

A.S. Kulharya ${ }^{\text {a, b }}$, V.S. Tonk ${ }^{c}$, J.N. Myers Jr ${ }^{\text {b }}$

aOncoMetrix, Poplar Health Care, Memphis, Tenn.,

bDivision of Dermatology, Georgia Health Sciences University,

Augusta, Ga., 'Department of Pediatrics, Texas Tech University

Health Sciences Center, Lubbock, Tex., USA

Cutaneous mosaics are an excellent reflection of genetic mosaicism. One such example of pigmentary mosaicism is phylloid hypomelanosis characterized by congenital hypochromic macules appearing as round or oval patches resembling asymmetrical leaves or oblong lesions. These patients also exhibit developmental delay and/or mental retardation in association with mosaic trisomy 13. A patient with phylloid hypomelanosis was recently reported with mosaic tetrasomy $13 \mathrm{q}$. We present a second such case with mosaicism for partial tetrasomy 13q further expanding and demonstrating the consistency of phenotype in these patients.

The patient is a 6-year-old black female with congenital hypopigmented macules without any delayed developmental milestones. She has mild dysmorphic features with tall forehead, thick eyebrows, hypertelorism, broad nasal bridge, malposition of teeth, and prominent central incisors with diasthema. She has bilateral clinodactyly of the 5th digits. She has leaflike and oval hypopigmented macules and patches on her trunk and extremities. Oblong hypopigmented patches are present on her lower extremities in a linear fashion that do not follow Blaschko's lines. She has history of bilateral strabismus, advanced bone age and premature adrenarche.

Cytogenetic analysis of 50 fibroblast cells from the hypomelanotic skin demonstrated 39 metaphase cells with 47 chromosomes with an extra copy of chromosome 13 . This cell line showed a derivative chromosome 13 with an inverted duplication with breakpoints at $13 \mathrm{q} 21.1$ and $13 \mathrm{q} 34$. The remaining 11 cells had a normal female karyotype. Similar results were observed from fibroblast cells from the phenotypically normal skin. FISH analysis confirmed the duplication of the $13 \mathrm{q} 34$ region with deletion of 13 q14 in the derivative chromosome 13. Array-CGH analysis on the cultured fibroblasts showed a gain of bands 13q21.1 to 13q34 spanning from $56,691,155-114,110,891$ base pairs, confirming the duplication and deletion in the derivative chromosome 13. Based on this information the karyotype is: $47, \mathrm{XX},+13, \operatorname{der}(13) \operatorname{dup}(13 \mathrm{q})$ $\operatorname{inv}(13)(q 21.1 q 34) \operatorname{del}(13)(q 12.1 q 14.3)[39] / 46, X X[11]$.

\section{4 \\ Interstitial 12q24.31 Deletion Detected by Array CGH in a Girl with Neonatal Hyperinsulinism, Childhood Diabetes Mellitus Type 2, Dysmorphism, Developmental Delay, and Behavioral Problems}

F. Li, K.J. Holbrook, X. Li, D.R. Witt

Department of Genetics, Kaiser Permanente San Jose Medical Center, San Jose, Calif., USA

A 12-year-old girl with obesity, diabetes, and developmental/ behavioral problems was evaluated. She was born at 37 weeks gestation with birth weight $3.8 \mathrm{~kg}$. Noted to have respiratory distress, hypoglycemia due to hyperinsulinism treated with Diazoxide, and patent ductus arteriosus. Subsequent physical examination found low-pitched weak cry, brachycephaly, arched eyebrows, anteverted nares, short neck with redundant skin, and significant developmental delay. Cardiology evaluation found absent proximal left coronary artery shunted surgically. At age 3 , she had only mild developmental delay. Glucose level had stabilized and Diazoxide was discontinued. Both weight and height remained at $>90 \%$ since then. She was diagnosed with diabetes mellitus type 2 at 8 years of age. Since age 11, she had anxiety, emotional outbursts, and self hurting behavior treated with Abilify. Karyotype was normal. FISH studies for Williams and cri-du-chat syndrome regions were normal. Array CGH was performed using the $8 \times 60 \mathrm{~K}$ human whole genome microarray (Agilent/ISCA/OGT) and revealed a $1.9 \mathrm{Mb}$ interstitial deletion within $12 \mathrm{q} 24.31$ at genomic coordinates 119,552,354-121,418,625. The deleted region contained 36 annotated genes and transcripts which included 23 OMIM genes. Mutations of some of the OMIM genes have been associated with disorders of glucose metabolism (HNF1A, $A C A D S$ ), development delay (ACADS, $H P D)$, and/or psychiatric problem (ACADS, $P 2 R X 7)$. A recent study reported that a female with a de novo deletion of 1.58 to $2.53 \mathrm{Mb}$ at similar breakpoints detected by BAC array CGH had a similar phenotype which includes hypoglycemia caused by hyperinsulinism, macroglossia, dysmorphism, and overgrowth as a neonate, and marked developmental delay and anxiety at age 11 . The deletion also contained $A C A D S, H N F 1 A, P 2 R X 7$, and HPD. As indicated in the literature, our case report provides further evidence that this deletion may represent a recurrent contiguous gene deletion syndrome.

Preprint Cytogenet Genome Res 136/4/2012 


\section{5}

Prenatal Detection and Characterization of a psu idic(8)(p23.3) Which Likely Derived from a Nonallelic Homologous Recombination between Two MYOM2 Repeats

Y.-C. Li ${ }^{\text {a, b }}$, S.-C. Chien ${ }^{\text {c, d , S.R. Setlur }}{ }^{f}$, W-D. Lin ${ }^{\text {d }, ~ F .-J . ~ T s a i ~}{ }^{d}$, C.-C. $\operatorname{Lin}^{\mathrm{b}, \mathrm{e}}$

aDepartments of Biomedical Sciences and Medical Research, Chung Shan Medical University and Hospital, b Graduate Institute of Basic Medical Sciences, Departments of ${ }^{\mathrm{C} O b s t e t r i c s}$ and Gynecology, dMedical Genetics, and ${ }^{\mathrm{e}}$ Medical Research, China Medical University and Hospital, Taichung, Taiwan; fDepartment of Pathology, Brigham and Women's Hospital, Boston, Mass., USA

Mosaicism with an isodicentric 8 with a breakpoint at p23.3 [idic(8)(p23.3)] is very rare. We report the first prenatal case on a male fetus in which obstetric ultrasound revealed multiple congenital anomalies at 28 weeks of gestation. Cytogenetic analysis of amniocytes showed mos 45,XY,-8,psu idic(8)(p23.3) [16]/46,XY,psu idic(8)(p23.3)[4] and that of cord blood lymphocytes revealed mos 46,XY,psu $\operatorname{idic}(8)(\mathrm{p} 23.3)[37] / 45, \mathrm{XY},-8, \mathrm{psu}$ $\operatorname{idic}(8)(\mathrm{p} 23.3)[13]$. FISH studies revealed that the break-reunion occurred at the cytoband 8p23.3 within the physical position 2.08 $\mathrm{Mb}$ from the $8 \mathrm{p}$ telomere. Chromosomal microarray analyses further assigned the duplication/deletion breakpoint at $2.16 \mathrm{Mb}$ (Agilent $244 \mathrm{~K}$ ) and at $2.19 \mathrm{Mb}$ (Affymetrix SNP6.0). Analysis of microsatellite DNA indicated that the psu idic(8)(p23.3) was derived from the maternal chromosome 8 . Together, these findings indicate that the fetus was nullisomic for $\sim 2.2 \mathrm{Mb}$ from 8 pter, trisomic for the rest of chromosome 8 in mosaic condition and likely had breaks in MYOM2 repeats of the maternal chromosome 8.

\section{6 \\ Copy Number Changes and Phenotypes Associated with Seizures in a Cohort Analyzed by High Density Whole Genome Array}

L. Matyakhina, J.M. Meck, S. Warren, S. Aradhya

GeneDx, Gaithersburg, Md., USA

Seizures are the result of hypersynchronous electrical discharges of cortical neurons. A wide variety of seizures are classified based on the location and extent of the abnormal discharge. The occurrence of at least 2 unprovoked seizures more than 24 hours apart is classified as epilepsy. Seizures often present within a broader syndromic phenotype. A genetic etiology underlies epilepsy in $\sim 40 \%$ of individuals. In our experience with over 10,000 cases of clinical whole genome array cases, 858 individuals had a referral indication which included seizures. Virtually all seizure patients referred for microarray testing had additional phenotypic abnormalities. The most frequent associated clinical features were: developmental delay/intellectual disability (92\%), heart defects (39\%), dysmorphic features (32\%), micro/macrocephaly (21\%), and hypotonia (16\%). We found that $25 \%$ (211 cases) of these cases had a copy number change (CNC) which was categorized as either 'pathogenic' or 'VUS' (variant of unknown significance); most often these were single aberrations. Seventy seven percent were interpreted as pathogenic CNCs, while $23 \%$ were categorized as VUS. We examined these cases to determine if there are some aberrations which occur more frequently. In 7 cases (3\%) the imbalances were due to chromosomal aneuploidy, generally involving the X chromosome. Approximately $27 \%$ of cases represented classic microdeletion syndromes involving chromosome regions such as 1q21.1 (TAR), 4p (Wolf-Hirschhorn), 5q35 (Sotos), 15q11.2q13 (Angelman/Prader Willi), 16p11.2, 17q21.31, 22q11.2 (DiGeorge/VCFS). Some aberrations included genes that have a known association with epilepsy, such as $N R X N 1, U B E 3 A$, MECP2, GABRA1, GABRG2, CHRNA, CASK, GPR98, SLC2A1, $D M D, N F 1$ and TSC2. We will highlight cases with small de novo aberrations containing genes with a possible role in seizure etiology.

\section{7}

\section{Epigenetic Exception of X Inactivation Reversal during Gametogenesis}

F. Nouraei ${ }^{\mathrm{a}}$, N.Rao ${ }^{\text {b }}$, B. Rashidi ${ }^{\text {C, I. Filip }}{ }^{\mathrm{d}}$, A. Radfar ${ }^{\mathrm{e}}$

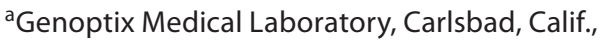

bUCLA Cytogenetics Laboratory, Los Angeles, Calif.,

'Affiliated Pathologist Medical Group, Torrance, Calif.,

${ }^{\mathrm{d}}$ School of Public Health, University of Massachusetts,

Amherst, Mass., e Department of Laboratory Sciences and

Nutrition, University of Massachusetts, Lowell, Mass., USA

False positive results in interphase fluorescence in situ hybridization (FISH) for the rapid prenatal detection of aneuploidy have been reported in literature. These case studies revealed a single signal using $\mathrm{X}$ and $\mathrm{Y}$ chromosome alpha-satellite probes which was initially interpreted as monosomy for the $\mathrm{X}$ chromosome in the fetus.

Due to advanced maternal age, the amniotic fluid of a 36-yearold woman G1P0 at 17 weeks of gestational age has been submitted for cytogenetic laboratory studies. The result of conventional cytogenetic studies (female chromosome analysis) on amniotic fluid was not concordant with the FISH results. FISH study on amniotic fluid showed monosomy $\mathrm{X}$ which is suggestive of the clinical diagnosis of Turner's syndrome. Ultrasound study, on the other hand, showed a normal fetal movement and cardiac activity. Since only 1 copy of X chromosome was identified in the interphase cells, parental chromosome analysis and FISH studies with the $\mathrm{X}$ and $\mathrm{Y}$ chromosome probes were performed.

Chromosome analysis of cultured peripheral blood lymphocytes from the parents revealed a normal 46,XY karyotype in the father and an apparently normal 46,XX karyotype in the mother. Hybridization of metaphase spreads from the mother with the $\mathrm{X}$ alpha satellite probe yielded the same results as the fetus.

The extended cytogenetic analysis of the maternal grandparents' peripheral blood revealed presence of $2 \mathrm{X}$ chromosomes by FISH and chromosome analysis in the grandmother. Interestingly, the maternal grandfather's FISH study showed only a single $\mathrm{Y}$ chromosome and a normal XY pattern in chromosomal analysis. 
Further X-inactivation studies on all submitted specimens showed skewed $\mathrm{X}$ inactivation toward the $\mathrm{X}$ chromosome with the nonvariant centromere.

The familial nature of these findings disclosed that skewed inactivation of the non-variant $\mathrm{X}$ chromosome is inherited in consequent generations. This variation and/or reduction, however, would not affect the centromere function.

\section{8 \\ Meiotic Segregation Pattern and Chromatin Structure Analysis of Spermatozoa from a Carrier with Complex Chromosome Rearrangement (CCR) t(6;10;11)(q25;q24;q23) - Case Report}

M. Olszewska ${ }^{a}$, N. Huleyuk $^{\text {b }}$, D. Zastavna ${ }^{\text {b }}$, E. Wiland ${ }^{a}$, M. Kurpisz $^{\text {a }}$

${ }^{a}$ Department of Reproductive Biology and Stem Cells, Institute of Human Genetics Polish Academy of Sciences, Poznań, Poland; ' Institute of Hereditary Pathology, Ukrainian Academy of Medical Sciences, Lviv, Ukraine

Complex chromosome rearrangements (CCR) are structural aberrations involving more than 2 breakpoints on 2 or more chromosomes. CCRs are a potential factor of high rate chromosomal imbalances leading to drastic decrease of male fertility, even reaching the spermatogenesis arrest. It is due to the complex meiotic configuration of hexavalent structures leading to production of 64 theoretical meiotic segregants. So, the presence of CCR in spermatozoa is very rare and they may appear de novo or be of parental origin. At this time, only 4 reports with examination of meiotic segregation in CCR were published. Percentage of unbalanced chromosome complements ranged between $69.4 \%$ and $86.5 \%$.

The purpose of the study was to determine the meiotic segregation pattern in spermatozoa of a normozoospermic balanced CCR carrier $\mathrm{t}(6 ; 10 ; 11)(\mathrm{q} 25 ; \mathrm{q} 24 ; \mathrm{q} 23)$ mat and sperm chromatin structure. Using FISH with combination of directly labeled probes for centromeres (10cen: red and blue, 11cen: red and green) and subtelomeres (6q red, 10q green, 11q blue) (Kreatech, NL), characteristics of chromosomes involved in CCR (also mFISH analysis; MetaSystems) and meiotic segregation patterns (in over 1,500 sperm cells) were examined. Also aneuploidy levels of chromosomes $13,15,18, \mathrm{X}$ and $\mathrm{Y}$ were assessed ( $\alpha$-satellite and whole chromosome painting probes). For sperm chromatin structure analysis, the deprotamination level of spermatozoa was evaluated by chromomycin A3 (CMA3) and aniline blue (AB) stainings on more than 1,000 sperm cells. Results obtained from CMA3 showed deprotamination in $20.0 \%$ of spermatozoa, for $A B$ in $16.5 \%$, and no statistical significance compared to control results $(21.7 \%$ and $15.9 \%$, respectively) was found. We may speculate that the high frequency of unbalanced spermatozoa produced in meiosis because of the hexavalent configuration, can be one of the causes of infertility revealed in that CCR carrier.

\section{9}

\section{Identification of a Novel Recurrent Microdeletion at 2q11.2 Associated with Dysmorphic Features and Developmental Delay}

S. Sampath ${ }^{\text {a }}$ P. Hixson ${ }^{\text {a }}$, P. Stankiewicz ${ }^{\text {a }, ~ S . W . ~ C h e u n g ~}{ }^{\text {a }}$, P.I. Bader ${ }^{\text {b }}$, A. Patel ${ }^{\text {a }}$

aDepartment of Molecular and Human Genetics, Baylor College of Medicine, Houston, Tex., bParkview Health Systems, Fort Wayne, Ind., USA

We report a recurrent deletion at 2 q11.2 spanning $\sim 900 \mathrm{~kb}$ that was identified using aCGH in 3 individuals from approximately 15,000 cases evaluated by our diagnostic center. The custom array targets all exons with significant backbone coverage, specifically avoiding regions of low-copy repeats (LCRs). Two of the three were sibs who inherited the deletion from an affected mother; the third case was a de novo deletion.

All individuals with the $2 \mathrm{q} 11.2$ deletion shared distinctive dysmorphic features including a prominent central forehead, down sloping eyes with a small eye opening, medial flare of the eyebrows, short flat philtrum, malar hypoplasia, prominent ears and mandible, large hands and feet, and inward turning of the feet, all reminiscent of an overgrowth syndrome. In addition, behavioral disorders of $\mathrm{ADHD}$, mood, anxiety, poor adaptive behavior, and expressive language defects were encountered. In the de novo 2 q11.2 deletion case, dyspraxia and autism spectrum disorder were also diagnosed.

The DGV database does not contain deletions or duplications spanning this interval. In addition, pathogenic deletions and duplications overlapping our $900 \mathrm{~kb}$ interval have been reported in the ISCA database. Several direct and indirect LCR flank the 900 $\mathrm{kb}$ deletion and we have identified 4 large direct LCRs (35 kb, 105 $\mathrm{kb}, 42 \mathrm{~kb}$ and $37 \mathrm{~kb}$ ) as potential candidates for mediating these deletions via NAHR. We are currently performing long-range PCR to map the deletion interval. The $\sim 900 \mathrm{~kb}$ interval contains 21 RefSeq genes and 1 unknown transcript. We discuss the role of these genes in the light of the phenotype seen in these patients. Although copy number changes spanning this interval have been reported before, this is the first report to include clinical correlates associated with 2 q11.2 deletion.

\section{0 [Withdrawn] \\ Further Characterization of the Critical Region of the 2p15-p16.1 Microdeletion Syndrome}

\author{
J. Song a , R. Lozano a , E. Spiteri ${ }^{\mathrm{a}}$, R. Schreck ${ }^{\mathrm{a}, \mathrm{b}}$, W.R. Wilcox ${ }^{\mathrm{a}, \mathrm{b}}$ \\ ${ }^{a}$ Medical Genetics Institute, Cedars-Sinai Medical Center and \\ bepartment of Pediatrics, UCLA School of Medicine, \\ Los Angeles, Calif., USA
}

Analysis of an oligonucleotide array comparative genomic hybridization $(\mathrm{aCGH})$ on a 2 -year-old patient with developmental delay, microcephaly and minor dysmorphic features showed a de novo deletion at 2p15-p16.1 with a maximum size of $1.11 \mathrm{Mb}$, which is the region involved in a newly recognized microdeletion syndrome. The candidate region for developmental delay and cra-

Preprint Cytogenet Genome Res 136/4/2012 
niofacial dysmorphisms has been previously assigned to 2 segments of the region (2.1 Mb and $570 \mathrm{~kb}$, respectively), based on reports consisting of 6 individual cases. A recently reported seventh case with a $3.5 \mathrm{Mb}$ deletion does not contain the $570 \mathrm{~kb}$ region and shares only a non-genic portion of the common $2.1 \mathrm{Mb}$ segment. We compared our patient with the 7 cases described previously and propose that there are 3 segments of interest present in this region. The most proximal segment of $190 \mathrm{~kb}(\mathrm{Ch}: 2: 61.13-$ 61.32 ) is shared by 7 out of 8 cases and contains 4 genes, KIAA1841, C2orf74, AHSA2 (activator of heat shock 90 Da protein) and USP34 (ubiquitin-specific protease 34). USP34 regulates Wnt/ $\beta$ catenin signaling which plays a critical role in brain development and many other developmental aspects. The next segment of 910 $\mathrm{kb}$ contains many genes including PEX13, REL and BCL11, which are shared by 6 out of 8 cases, and this region may contribute to some of the additional clinical features. The most distal segment of $1.09 \mathrm{Mb}$ contains no genes, and is shared by 6 out of 8 cases. The pathogenic effect of this distal segment may be mediated by positive or negative regulation of other genes in the 2p15-p16.1 region. In summary, our finding provides a better understanding of the genetic complexity of the clinical phenotypes of the $2 \mathrm{p} 15$ p16.1 microdeletion syndrome.

\section{1 \\ Unexpected Abnormal Maternal Karyotype Suggests a Mechanism of Meiotic and Mitotic Errors Resulting in Tetrasomy $X$ in a Patient with Developmental Delay and Dysmorphic Features}

F.C. Wheeler ${ }^{\mathrm{a}}$, J. Pfotenhauer ${ }^{\mathrm{b}}$, J.A. Phillips $1 \mathrm{II}^{\mathrm{b}}$

${ }^{a}$ Department of Pathology and Laboratory Medicine,

${ }^{b}$ Department of Pediatrics, Vanderbilt University Medical

Center, Nashville, Tenn., USA

Here we present the case of a 16-month-old girl with developmental delay, hypotonia and mildly dysmorphic features referred to the Genetics clinic with a suspicion of Down syndrome. Physical examination revealed plagiocephaly, a flat nasal bridge, widely spaced eyes with slight epicanthal folds, increased nuchal skin, small ears, single palmar creases and 5 th finger clinodactyly. Both parents are intellectually impaired. Chromosome analysis revealed tetrasomy X $(48, \mathrm{XXXX}$, tetraX) in every cell examined. Tetrasomy $\mathrm{X}$ is a rare disorder characterized by a highly variable phenotype that can range from mild to very severe, but usually includes some degree of intellectual deficit. TetraX has been reported to arise from successive maternal non-disjunction (NDJ) events in meiosis I and II with the child inheriting 3 (or 4) X chromosomes from the mother. Maternal chromosome studies and SNP microarray analysis were performed in order to determine the origin of the child's two extra X's. The patient's mother was found to have an abnormal karyotype with an apparently balanced X;16 translocation [46,X,t(X;16)(p13.1;p11.2)]. The mother's abnormal derivative $\mathrm{X}$ was not present in the patient, excluding the possibility of a maternal meiosis I NDJ event and suggesting meiosis II NDJ followed by an early post-zygotic error. SNP genotypes generated from microarray analysis showed homozygosity for markers surrounding the centromere, further support- ing a mechanism of errors in meiosis II and mitosis. TetraX was an unexpected finding in our patient, reinforcing the importance of laboratory confirmation of suspected DS. The patient's and mother's clinical and cytogenetic findings alter her prognosis and recurrence risks. A previously published case report combined with these current findings demonstrates that mechanisms other than successive meiotic errors are capable of generating an individual with extra X chromosomes. Monitoring this patient's growth and development will add to the limited body of knowledge about TetraX.

\section{Poster Presentations Non-Human Cytogenetics}

\author{
62 \\ Chromosome Abnormalities Identified in a Lowland \\ Gorilla Pedigree \\ M.L. Houck ${ }^{\text {a }}$ S.J. Charter a , J.A. Fronczek ${ }^{\text {a }}$, M. Domingos a \\ K. Helmick ${ }^{\text {b }}$, T. Hermann ${ }^{\text {b }}$, G. Mastromonaco ${ }^{\text {c }}$, O.A. Ryder ${ }^{\text {, }}$, \\ N.C. Hawkes ${ }^{b}$ \\ anstitute for Conservation Research, San Diego Zoo Global, \\ Escondido, Calif., b'Woodland Park Zoo, Seattle, Wash., USA; \\ ${ }^{C}$ Reproductive Physiology, Toronto Zoo, Toronto, Ont., Canada
}

Cytogenetic analyses are not routinely performed on great apes and thus relatively little is known about the prevalence of chromosomal variability and abnormalities among this group. Multiple spontaneous abortions and congenital malformations in offspring of several lowland gorillas (Gorilla gorilla) at Woodland Park Zoo, Seattle, led to karyotype analysis of animals in the extended pedigree. Fibroblast cell lines were established for most of the individuals in the study and accessioned into the San Diego Zoo's Frozen Zoo ${ }^{\circledR}$. Heteromorphisms are known to occur at high frequency in the gorilla karyotype and were noted in pair 15 and 17 in many of the living individuals of this family. In addition, an apparent deletion of a single band at the terminal end of HSA9q was found in 1 female. The most notable finding was trisomy of HSA15 in fetal material obtained from one of the spontaneous abortions from a dam with advanced maternal age, 'Amanda'. Trisomy 15 in humans is linked to maternal age and is associated with spontaneous abortion. The next offspring of Amanda was a female born with spina bifida and no chromosome polymorphisms were detected in her karyotype. Most neural tube defects are thought to have multifactorial inheritance including both genetic (including single-gene defects) and environmental causes. Continued cytogenetic analyses of both captive-bred and wild gorillas will contribute to our understanding of genetic disorders in this species and is an important component of captive gorilla breeding programs. Utilization of a high quality assembly of a gorilla genome sequence will allow more detailed analysis of structural variation and association with reproductive and developmental abnormalities. 


\section{3 [Withdrawn]}

\section{Cytosystematics of African Gerbils (Rodentia, Muridae, Gerbillinae)}

\section{Knight}

Evolutionary Genomics Group, Department of Botany and

Zoology, University of Stellenbosch, Stellenbosch, South Africa

Gerbillinae is comprised of 16 recognised genera and 112 species. They have a global distribution that extends across arid regions in Africa, Asia and the Middle East. Since their divergence more than 10 mya, the phylogenetic relationships within the group have remained contentious. Systematic studies involving morphology (dental, cranial and post-cranial), genes (cyt b, $12 \mathrm{~S}$ rRNA, 16S rRNA), conventional cytogenetics (G-, C- and Rbanding) and allozymes have revealed conflicting results. The current examination of gerbil phylogenetics involves molecular cytogenetics, which aim to contribute or aid in elucidating their taxonomic relations. Chromosomes and chromosomal rearrangements constitute characters which may be used cladistically to construct phylogenies. The study employed G- and C-banding and ZOO-FISH. Gerbillurus paeba $(2 \mathrm{n}=36)$ fibroblast cell culture was flow-sorted and painted onto a representative sample size of 7 genera and 11 species. The most recent phylogeny of Gerbillinae by Chevret and Dobigny [2005] included an analysis of complete cyt b and $12 \mathrm{~S}$ rRNA genes on representative species from 11 genera. The molecular-based study retrieved 3 main clades and 5 lineages. ZOO-FISH analyses, or cross-species chromosome painting, provided further support of these lineages. The mode and tempo of chromosome rearrangements were found to be more dominant in certain groups of taxa. Within the Gerbilliscus and Gerbillurus genera for instance, the most common rearrangements were Robertsonian translocations. Rearrangements became complex in more distantly related species and lineages. The Taterillus lineage, represented by T. pygargus, was dominated by tandem fusions, while chromosome evolution in Psammomys and Meriones were predominantly driven by fissions and inversions. Whole arm reciprocal translocations were also observed.

Cross-species chromosome painting also found an entirely Cpositive autosome in G. paeba to have whole chromosome homology in all examined specimens. The role of chromosome rearrangements and chromosome evolution in this dynamic group of rodents will be discussed.

\section{4}

\section{Trisomy-Associated Hydrops Fetalis in a Horse Fetus}

T.L. Lear ${ }^{\mathrm{a}}$, J. Lundquist ${ }^{\mathrm{a}}$, J.A. Fronczek ${ }^{\mathrm{b}}$, S.J. Charter ${ }^{\mathrm{b}}$, T.V. Little ${ }^{\mathrm{c}}$

${ }^{a}$ Maxwell H. Gluck Equine Research Center, University of Kentucky, Lexington, Ky., ' Institute for Conservation Research, San Diego Zoo Global, Escondido, Calif., 'Adena Springs Farm, Paris, Ky., USA

Autosomal trisomy has rarely been identified in the horse and all cases have involved the smallest acrocentric chromosomes in the horse karyotype. In addition, no lethal fetal autosomal tri- somy has been identified in the horse. While a hydrops fetalislike condition has been observed in horse fetuses, no affected fetus has ever been recovered and karyotyped. Herein, we describe an apparent case of trisomy-associated hydrops fetalis in a horse fetus.

At 28 days post-ovulation, a 6-year-old Thoroughbred mare was examined by ultrasound to determine the status of the fetus. The fetus appeared normal. However, during a fetal gender exam on day 62 , the fetus was observed to be grossly abnormal. The male fetus exhibited multifloculated fluid accumulation in the nuchal and cervical regions with marked subcutaneous edema extending caudally to the abdominal region. Serial ultrasound examinations revealed increasing pleural effusion and ascites. The fetal heartbeat was lost on about day 76 post-ovulation and the fetus was extracted. The fetus exhibited severe subcutaneous edema and redundant skin around the neck, thorax and abdomen. Fibroblast cells derived from several fetal organs were analyzed by GTGbanding and FISH. Fetal karyotype analyses revealed pure trisomy 26. FISH with horse chromosome 26-specific probes confirmed the trisomy.

Horse chromosome 26 shows conserved homology to human chromosome 21 including the Down Syndrome Critical Region. Human hydrops fetalis has been confirmed in cases of trisomy 21 . Only 1 living horse with mild congenital abnormalities and trisomy 26 has been reported. We report the first case of trisomy 26 and associated hydrops fetalis in a horse fetus. Since the hydrops fetalis-like condition is occasionally observed in horse fetuses at this stage of gestation, trisomy 26 may be more prevalent in horses than previously recognized and may contribute to early fetal losses observed in this species.

\section{5}

\section{A Novel Cervid Satellite DNA Originated from a Putative Ancient Centromeric DNA with CDEI-Like Repeated Sequences}

\author{
C.C. Lin ${ }^{\mathrm{a}}$, L. $^{-J . H \text { sieh }^{\mathrm{b}} \text {, Y.-M. Cheng }}{ }^{\mathrm{c}}$, Y.-C. Li ${ }^{\mathrm{a}}$, b
}

${ }^{a}$ Graduate Institute of Basic Medical Science, China Medical University and Department of Medical Research, China Medical University Hospital, and Institute of Medicine, Chung Shan Medical University, bDepartment of Biomedical Sciences, Chung Shan Medical University and Department of Medical Research, Chung Shan Medical University Hospital, 'Department of Agronomy, National Chung Hsing University, Taichung, Taiwan

It has been proposed that pericentromeric satellite DNA arises from the progressive proximal expansion of ancient centromeric DNA. To recover putative ancient centromeric DNA, we microdissected the pericentromeric/centromeric DNA from an $\mathrm{X}+3$ centromere of Indian muntjac (Muntiacus muntjak vaginalis) and constructed a microclone-library of $\mathrm{X}+3$ centromeric DNA. A new satellite DNA element, designated as satellite VI, was isolated from this library. FISH studies revealed that satellite VI is predominately located on the distal pericentromeric region of the Indian muntjac $\mathrm{X}+3$ chromosome. Its sequences are organized as $11 \mathrm{bp}$ monomeric tandem repeats. The first 8 nucleotides of the monomeric consensus sequences are identical to the CDEI ele- 
ment in the centromere of the budding yeast Saccharomyces cerevisiae. Therefore, we speculate that this newly found satellite DNA is a vestige of the putative ancient centromeric DNA with basic repeat unit similar to the yeast CDEI element.

\section{6 \\ G- and C-Banded Metaphase Karyotypes in the Chinchilla (Chinchilla laniger) \\ H. Pang ${ }^{\text {a }}$ S. Li ${ }^{\text {a }}$, T. Seale ${ }^{\text {b }}$ \\ ${ }^{a}$ Genetics Laboratory, Department of Pediatrics, \\ ${ }^{b}$ Department of Biological Psychology, University of Oklahoma \\ Health Sciences Center, Oklahoma City, Okla., USA}

The chinchilla is an important animal model used in clinical research relevant to prevention and/or treatment of human middle and inner ear infections (otitis media and otitis internal) and development of surgically implantable devices to remedy hearing loss. Lack of a standardized karyotype for the chinchilla has precluded genomic sequencing and transcriptomic approaches to the identification of host genes with important roles in middle and inner ear diseases and their treatment. In this study we used GTGand CBG-banding techniques to better describe the chinchilla karyotype. Chromosome preparations from both adult male and female purpose-bred chinchillas were obtained from whole blood lymphocytes. After G-banding, about 450 band-level chromosomes were identified and classified according to the International System for Cytogenetic Nomenclature of Domestic Animals. In each of 14 chinchillas we found that the diploid number was 64 . Most of the autosomes were metacentric. The X chromosome, which has a C-positive band in the $\mathrm{p}$ arm in addition to the centromere, is the largest chromosome. The $\mathrm{Y}$ chromosome is a minute acrocentric. This is the first attempt to use current, well defined, karyotyping methods to systematically identify individual chinchilla chromosomes based on G- and C-banding patterns.

\section{7}

\section{Cytogenetic Analysis of Anuran Species of Akure, Ondo State (South-Western Nigeria)}

\author{
A.A. Sorungbe ${ }^{\mathrm{a}}$, I. Awopetu ${ }^{\mathrm{b}}$ \\ aUnit of Cytogenetics, Department of Biology, Federal \\ University of Technology, Akure, ${ }^{b}$ Department of Zoology, \\ Obafemi Awolowo University, lle-Ife, Nigeria
}

A detailed characterization of the karyotypes of some anuran species found in Akure, Ondo State (south-western Nigeria) was carried out. It involved cytogenetic studies using conventional Giemsa staining, C-banding and silver staining. Parameters determined for each species investigated include diploid number (2n), fundamental number, presence/absence of nucleolar organizer regions (NORs) as well as other structural aberrations. The karyotypes obtained were based on the chromosomes' relative length, centromeric position and arm (p/q) ratio. Ag-staining techniques revealed presence of nucleolar organizer regions, while C-banding enabled the correct pairing of homologues. Some of the species cytogenetically analyzed include Bufo regularis, Haplobatrachus occipitalis, Kassina senegalensis, Hyperolius sp., Plychadena aequiplicata and Afrixalus sp. A detailed karyogram was established for each species from the results obtained.

\section{8 [Replaces platform presentation 12] Polymorphism in the Location of Silver-Stained Nucleolus Organizing Regions and the $18 \mathrm{~S}+28 \mathrm{~S}$ Ribosomal Genes in North American Hylid Frogs}

\section{J.E. Wiley, H.S. Bonner}

The Brody School of Medicine, East Carolina University, Greenville, N.C., USA

Most anuran amphibians have a single 'standard' pair of nucleolus organizing regions (NORs) in their karyotype, as determined by silver staining, but occasionally NORs are seen at additional locations. Sometimes the standard location of the NORs in anuran species differs between or within populations. The North American hylid frog Hyla chrysoscelis has been reported to have differences in the standard location of the NORs as well as additional NOR locations in the karyotype. Here we report the results of fluorescent in situ hybridization (FISH) with a DNA probe to the $18 \mathrm{~S}+28 \mathrm{~S}$ ribosomal (rDNA) genes, which are located at the NORs, and silver staining of the chromosomes of some North American hylid frogs. All of the species studied had additional rDNA locations, and in $H$. chrysoscelis the additional sites were often numerous. A total of 30 individuals of $H$. chrysoscelis were studied and no two had an identical pattern of rDNA hybridization. However, some of the additional locations of the rDNA are common to populations that are widely separated geographically. Most of the extra rDNA locations in the species examined were not detectable by silver staining, except in H. squirella, where no 2 individuals out of 10 studied were identical by FISH or silver staining. It has been demonstrated that the additional $18 \mathrm{~S}+28 \mathrm{~S}$ rRNA genes have been precisely inserted into their locations without any rearrangement of the chromosomes. One possible explanation for the observations presented here is that transposons are involved in the apparent mobility of the rDNA in the genomes of these species. 


\section{Poster Presentations Cancer Cytogenetics}

\author{
69 \\ Investigation of Chromosomal Aberrations in \\ Hepatocellular Carcinoma Patients as Detected by \\ Fluorescence in situ Hybridization \\ M.A. Al Abboud a , M.S. Aly ${ }^{\mathrm{a}, \mathrm{b}}$ \\ aBiological Science Department, Faculty of Science, \\ Jazan University, Jazan, Saudi Arabia; 'boology Department, \\ Beni-Suef University, Beni-Suef, Egypt
}

Hepatocellular carcinoma (HCC) is the sixth most common malignancy and the third most common cause of cancer deaths worldwide. The major risk factors include chronic viral infection (HBV and $\mathrm{HCV}$ ), alcoholic/nonalcoholic liver disease, environmental carcinogens (i.e. aflatoxin B1 (AFB)), and inherited genetic disorders (Wilson's disease, hemochromatosis, $\alpha$-1-antitrypsin deficiency, and tyrosinemia). With a few exceptions, HCC always develops in the setting of chronic hepatitis or cirrhosis, in which there is continuous inflammation and regeneration of hepatocytes. The non-random accumulated genetic alterations or chromosomal aberrations during the processes of inflammation, regeneration, and cirrhosis lead to the development of HCC. This multi-step process starts from hyperplastic change, dysplasia, and early HCC, eventually resulting in full-blown HCC. Qualitative and quantitative genetic alterations precede each step of carcinogenesis. The main aim of this study was to evaluate the genetic aberrations of different chromosomes in HCC patients. Interphase cytogenetics with a panel of centromeric probes was performed on paraffin embedded HCC samples. The most common chromosomal aberrations detected were gain of chromosomes 8 and 17. Loss of Y chromosome and monosomy 4 were also detected. Negative correlation could be detected between chromosome 4 and 8 . Negative correlation was also detected between chromosome 1 and cirrhosis. In conclusion, gains and losses of DNA found in this study, probably involve oncogenes and tumor suppressor genes that play a role in the puzzle of hepatocarcinogenesis.

\section{0}

\section{Polysomy 17 as Predictor of HER2 Gene Overexpression in Breast Cancer Patients}

O. Al-Abdulwahed ${ }^{\mathrm{a}-\mathrm{C}}$, B. McMahill ${ }^{\mathrm{a}}$, Y. Zou $^{\mathrm{a}}$, R. Saleki ${ }^{\mathrm{a}}$

apathology Associates Medical Laboratories, PAML, Spokane, Wash., ${ }^{b}$ Providence Sacred Heart Medical Center, Spokane, Wash., USA; 'King Fahad Specialist Hospital, Dammam, Saudi Arabia

Background: Predictive and prognostic markers are increasingly utilized to guide therapy selection and accurate identification of the HER2 gene amplification and/or overexpression status in breast cancer patients. The American Society of Clinical Oncology (ASCO) and The College of American Pathologist (CAP) in its latest published clinical practice guidelines provided an algorithm of testing for additional assessment of equivocal results obtained by either FISH or IHC method. Here, we present our experience in the clinical diagnostic laboratory in which FISH assay indicates the presence of multiple copies of chromosome 17 (polysomy 17) in FFPE breast tissues.

Methods: Retrospective study of all FFPE breast cancer tissues assessed by FISH for HER2 gene amplification in our lab in the last 4 years. Based on the current HER 2 testing algorithm, all cases are initially assessed by FISH, and, based on the literature test guidelines, cases with equivocal results (HER2/CEP17 ratios between 1.8-2.2) and cases with evidence of polysomy 17 were subjected to IHC. We define polysomy 17 as $20 \%$ of cells showing more than 4 copies of both CEP17 and HER2.

Results: From a total of 1277 FISH assays, 168 (13.2\%) were positive for HER2 gene amplification, 1030 (80.7\%) were negative and 79 cases had equivocal results or showed evidence of polysomy 17. From this latter category, 23 had evidence of polysomy 17. Follow-up assessment of HER 2 protein expression status by IHC showed positive results for HER2 overexpression in 8 cases (35\%), and only in $10 \%$ of equivocal cases.

Conclusions: In breast cancer tissues polysomy 17 may result in overexpression of HER2 protein. Evidence of polysomy $17 \mathrm{ob}-$ tained via FISH assay is a good indicator for overexpression of HER 2 gene and must be further evaluated by an alternate method such as IHC to confirm or rule out HER 2 gene overexpression.

71

Comparison of Genetic Changes in Transitional and Squamous Bladder Cancers

M.S. Aly ${ }^{\mathrm{a}, \mathrm{b}}$, H.M. Khaled ${ }^{\mathrm{c}}$

${ }^{a}$ Biological Science Department, Faculty of Science, Jazan University, Jazan, Saudi Arabia; ' $Z$ Zoology Department, BeniSuef University, Beni-Suef, 'Department of Medical Oncology, National Cancer Institute, Cairo University, Cairo, Egypt

In western countries, more than $90 \%$ of primary bladder carcinomas are transitional cell carcinoma (TCC), whereas squamous cell carcinoma (SCC) comprises less that 10\%. Carcinoma of the urinary bladder is the most common malignancy in many

Preprint Cytogenet Genome Res 136/4/2012 
tropical and subtropical countries due to endemic infection by Schistosoma hematobium. Compared with non-schistosome bladder cancer, schistosome-related bladder cancer has different clinical and pathological features. In this study, dual-color FISH cytogenetic analysis was performed using 2 oncogenes (HER-2/neu and $M C Y$ ) and a tumor suppressor gene ( $p 53)$ in relation to chromosomes 8 and 17 centromeres in a group of patients presenting with schistosomal associated squamous and transitional cell carcinoma. To the best of our knowledge, this is the first report to compare genetic alterations in both transitional and squamous subgroups of schistosomal bladder cancers in Egyptian patients using the FISH technique. Our data showed that different histologic subgroups of bladder tumors are characterized by distinct patterns of genetic alterations. The genetic changes found in the transitional cell group differ from tumors exhibiting squamous differentiation.

\section{2}

\section{A t $(8 ; 14)(q 24 ; q 32)$ Translocation Resulting in an Atypical /GH/MYC Genomic Fusion in Chronic Lymphocytic Leukemia}

P.H. Bui, I. Amarillo, B.S. Shackley, C.A. Tirado

Clinical Molecular Cytogenetics Laboratory, Department of Pathology \& Laboratory Medicine, David Geffen UCLA School of Medicine, Los Angeles, Calif., USA

Chromosomal translocation between chromosomes 8 and 14 involving $I G H$ and $M Y C$ is characteristic of Burkitt lymphoma, but it is rarely seen in chronic lymphocytic leukemia (CLL). Herein, we report the clinical, immunophenotypic, cytogenetic and molecular cytogenetic features of 1 unique CLL case with atypical $\mathrm{t}(8 ; 14)(\mathrm{q} 24 ; \mathrm{q} 32)$ translocation. Our patient, a 67-year-old male was diagnosed with CLL with numerous atypical lymphoid cells including prolymphocytes, smudge cells, normocytic normochromic anemia and thrombocytopenia. Co-expression of CD19, CD5, and CD23 was detected, and ZAP-70 expression was positive. Interphase FISH studies confirmed deletions of the $11 \mathrm{q} 22.3$ region containing the $A T M$ gene and $13 \mathrm{q} 14$. In addition, G-banding showed $\mathrm{t}(8 ; 14)(\mathrm{q} 24.1 ; \mathrm{q} 32)$ in $50 \%$ of the cells analyzed. Concurrent IGH-MYC FISH studies on metaphase cells not only confirmed the fusion IGH-MYC signal on the der(14) but also showed a der(8) with only 1 IGH signal. FISH using the CMYC break apart probe on metaphases also revealed a der(8) without any signal and a $\operatorname{der}(14)$ with a whole $C-M Y C$ signal translocated proximally to the $I G H$ gene. Similar structural rearrangements on $8 \mathrm{q}$ and $14 \mathrm{q}$ have been reported in some CLL cases. However, our case presents atypical breakpoints between these regions that do not activate $C-M Y C$ (confirmed by immunohistochemistry) which correlates with the present clinical scenario of this patient.

\section{3}

\section{A Rare Case of Acute Myeloid Leukemia with an $\operatorname{inv}(8)(p 11.2 q 13)$}

C. Henderson a , V. McDaniel a , D. Graham ${ }^{\text {b }}$, K. Maloney ${ }^{\mathrm{b}}$, L. Meltesen ${ }^{\text {a }}$, X. Liang ${ }^{\text {b }}$, K. Swisshelm ${ }^{\text {a }}$, L. McGavran ${ }^{\text {a }}$

${ }^{a}$ Colorado Genetics Laboratory, University of Colorado at

Denver, ${ }^{b}$ Children's Hospital Colorado, Denver, Colo., USA

Recurring chromosomal inversions, other than the inversion 16 , are rare in leukemia. We present the case of a 17-year-old female whose cytogenetic study revealed a 46,XX,inv(8)(p11.2q13) [20] acquired clone in the bone marrow. This patient presented with fatigue, fever and weight loss, leukocytosis and numerous circulating blasts. The bone marrow showed 90\% leukemic blasts. Flow cytometric analysis was positive for HLA-DR, CD11c, CD15, CD33, CD56, and CD45 (moderate). The final diagnosis was acute myeloid leukemia without maturation (WHO Classification) or AML-M1 (FAB Classification).

Interphase fluorescence in situ hybridization (FISH) studies using chromosome 8 centromere sequences (D8Z2) demonstrated an extra small signal. Metaphase FISH revealed a small D8Z2 signal in proximal chromosome $8 \mathrm{p}$, while the larger signal moves with the centromere to form a more metacentric-appearing inversion 8 .

The patient was treated with current best practice therapy (ARA-C, Daunorubicin, and Etoposide). At 1 month post induction the patient was in remission with no morphologic evidence of residual myeloblasts. Standard cytogenetics revealed a normal 46 , XX karyotype, and FISH studies showed no evidence of the previous abnormal chromosome 8 centromere signal.

The pericentric inversion 8 is a rare but described abnormality observed in acute myeloid leukemia, reported in fewer than 20 cases. This inversion has occurred predominantly in females at a relatively young age. This inversion results in the fusion of the MOZ (monocytic leukemia zinc finger protein) gene located on 8 p11.2 and the TIF2 (transcriptional intermediary factor) gene located on $8 \mathrm{q} 13$. The MOZ gene plays a key role in hematopoiesis. The MOZ-TIF2 fusion changes histone acetylation, resulting in a leukemogenic process. Careful correlation of FISH and standard cytogenetic studies are necessary to demonstrate this abnormality.

\section{4 \\ Evolution of an Isoderivative Chromosome 7q in t-AML following Treatment of Triple-Negative Breast Cancer}

\section{S.R. Horn, K. Nandi, K.L. Deak, C.W. Rehder, B.K. Goodman}

Department of Pathology, Duke University Medical Center, Durham, N.C., USA

Monosomy 7 and deletion $7 \mathrm{q}$ are common cytogenetic abnormalities in both de novo and therapy-related MDS and AML (tMDS, t-AML). Here, we report on a 59-year-old female, diagnosed with t-AML secondary to treatment of her stage IA breast cancer 3 years prior, which included a mastectomy, chemotherapy, and chest wall irradiation. 
On routine exam, she was found to be anemic, and a subsequent bone marrow biopsy showed multilineage dysplasia and $30 \%$ blasts, consistent with therapy-related AML (FAB M1/M2). Cytogenetic testing showed deletion within the long arm of chromosome 7 of the bands 7q22 through q34 in all metaphase cells examined. She began induction chemotherapy, followed by 2 rounds of consolidation therapy, and a nonmyeloablative haplomatch stem cell transplantation.

Within 1 month of transplantation, bone marrow FISH analysis showed the persistence of a clone with deletion 7q, although the corresponding pathology report indicated an acellular marrow without detectable hematopoiesis, and no dividing cells were available for chromosome analysis. She then underwent a second infusion from the same donor. Although later bone marrow biopsies showed the persistence of AML (46,XX,del(7)(q22q34)// $46, \mathrm{XY}$ by chromosome analyses, $7-8 \%$ deletion $7 \mathrm{q}$ by FISH, and $1-5 \%$ myeloid blasts by pathology reports), molecular engraftment analyses were encouraging, with donor allele compositions of 80-90\% (CD15+ and CD3+ fractions).

Following a period of persistent pancytopenia, repeat bone marrow analysis showed the formation of a rare isoderivative chromosome 7, which consisted of 2 mirror image copies of the long arm of the deleted 7q observed previously. The corresponding pathology report indicated a fibrotic marrow, with circulating blasts noted. Six weeks later, bone marrow cytogenetic analysis showed further evolution, with 2 new subclones present, one showing deletion 12p. In summary, chromosome and FISH analyses showed the persistence of low-level residual disease and provided evidence of clonal evolution, together warning of disease progression.

\section{5 \\ Correlating Genetic Alterations from Array Comparative Genome Hybridization and Next-Generation Sequencing with Histology and Clinical Outcome in Melanocytic Neoplasms \\ Y.J. Liu ${ }^{\text {a }, ~ Y . ~ Z h o u ~}{ }^{\text {a }}$ E. George ${ }^{\text {b }}$ \\ ${ }^{a}$ Cytogenetics and Genomics Laboratory, ${ }^{b}$ Dermatopathology, Anatomic Pathology, Department of Pathology, University of \\ Washington, Seattle, Wash., USA}

The incidence of melanoma in the United States is increasing rapidly and is about 1 in 70 currently. Due to limited systemic therapies for treating advanced stages of melanoma, early detection is the key to improve survival. Although particular melanoma subtypes are clinically and histopathologically distinct, they do not provide independent prognostic value. Molecular studies revealed some genetic defects of several genes and pathways in melanoma; however, the etiology of melanoma development is still largely unknown. The goal of this study is the use of highresolution, genome-wide approaches to identify genomic aberrations that contribute to melanoma transformation, development and progression and correlate these genetic findings with the histology and clinical outcome in hope of generating a molecular classification scheme for melanocytic neoplasms. The FFPE mel- anoma samples used in this study include malignant melanoma, metastasized melanoma, atypical nevi and benign nevi. We employed both whole genome scan using CCMC 180K CGH+SNP array and panel of actionable gene sequencing using next-generation sequencing in this study. The analyses and results of these samples will be presented and its clinical utility will be discussed.

\section{6 \\ Mismatched Crossing-Over between Sister Chromatids Yields Identical Duplication of the Proximal Segment and Deletion of the Distal Segment of IGH Gene but Phenotypic Expression Is Modulated to Mantle Cell Lymphoma or Chronic Lymphocytic Leukemia by the Presence of Additional Chromosome Abnormalities}

\section{N.S. Mitter}

Cytogenetics and FISH Laboratories, Dianon Systems (LabCorp), Shelton, Conn., USA

Most abnormalities of immunoglobulin heavy chain (IGH) gene in lymphoid and plasma cell disorders involve a rearrangement with another gene or deletion of the distal section of the gene through a simple terminal deletion $[\operatorname{del}(14 \mathrm{q})]$ or its replacement by a genetic region of indeterminate origin [add(14q)]. We report here 1 case each of chronic lymphocytic leukemia (CLL) and mantle cell lymphoma (MCL) wherein mismatched crossing over between sister chromatids resulted in identical duplication of proximal segment and deletion of distal segment detected by a combination of probes for the $I G H$ gene.

The break-apart probe for IGH gene by Abbott produces an orange signal for the very proximal 3' segment of IGH gene, and a green signal for the distal $5^{\prime}$ segment. This probe does not cover an intervening distal portion of the $3^{\prime}$ segment of IGH gene, while probe for $I G H$ gene in the mix with CCND1 or BCL2 probes covers the whole length of $I G H$ gene. With both these patients, the probe for $I G H$ in the mix indicated an extra signal for $I G H$ without gene rearrangement. The break-apart probe for IGH gene did not reveal an extra orange signal for the very proximal $3^{\prime}$ segment of IGH gene, while still showing a deletion of green signal for $5^{\prime}$ segment. These FISH results imply a duplication of only the intervening portion between $3^{\prime}$ and $5^{\prime}$ segments of IGH gene, with deletion of $5^{\prime}$ segment. Apparently, these abnormalities arose through mismatched cross-over between 2 IGH gene segments on sister chromatids and a resulting proliferation of cells with this combination of deleted-duplicated regions of IGH gene. The patient with MCL was still positive for cyclin-D1 amplification and the second patient had other abnormalities associated with CLL, resulting in a differential modulation of the same deletion-duplication abnormality of the $I G H$ gene. 


\section{7}

\section{Waiting for the Axe to Fall: Ph Chromosome in the Bone Marrow prior to Overt Clinical and Morphologic Evidence of Neoplastic Disease}

K. Ouyang ${ }^{\text {a }}$, M. Jamali ${ }^{\text {b }}$, G. Raca ${ }^{\text {a }}$, J. Anastasi ${ }^{\text {b }, ~ O . ~ B o r i n e t s ~}{ }^{\text {a }}$, T. Raoul ${ }^{\text {a }}$ T. Yessen ${ }^{\text {a }}$, V. Aggarwal ${ }^{\text {, J. J. Shay }}{ }^{\text {a }, ~ C . ~ R u b i n ~}{ }^{c}$

Departments of ${ }^{a}$ Medicine, ${ }^{b}$ Pathology, and ${ }^{\mathrm{c} P e d i a t r i c s,}$ University of Chicago, III., USA

Chronic myelogenous leukemia (CML) typically has a bi-or tri-phasic natural history, presenting in either indolent chronic phase (CP), accelerated phase (AP) or blast crisis (BP). In rare cases, patients present in $\mathrm{BP}$ with elevated white blood cell counts (WBCs), more than $20 \%$ circulating blasts in the peripheral blood or bone marrow, and symptoms related to severe cytopenias or splenic enlargement.

We report a case of a 14-year-old male initially presenting with no overt clinical or hematologic evidence of neoplastic disease, but showing the presence of the $t(9 ; 22)$ abnormality in the bone marrow. The typical $B C R-A B L 1$ fusion signal pattern was detectable by FISH in $\sim 18-30 \%$ of bone marrow cells (on different occasions), and real-time quantitative PCR (RQ-PCR) showed the presence of the p $210 B C R-A B L 1$ fusion transcript. Surprisingly, this patient rapidly progressed to CML in lymphoid BP (precursor B type) in the span of a month, as evidenced by his WBCs increasing from $4 \mathrm{k} / \mu \mathrm{l}$ to $74 \mathrm{k} / \mu \mathrm{l}$ within a week and his unequivocal bone marrow study. FISH analysis of this study revealed an extra $A B L 1$ signal in addition to the $B C R / A B L 1$ fusion in $\sim 80 \%$ of the bone marrow cells. Consistent with the FISH findings, cytogenetic analysis showed the presence of the $\mathrm{i}(9)(\mathrm{q} 10)$ abnormality, that was not seen in the initial sample. This case illustrates the utility of cytogenetics in diagnosing an incipient phase of CML in a patient lacking overt clinical and morphologic signs of neoplastic disease and the phenomenon of rapid progression and clonal evolution of lymphoid blast crisis in pediatric CML.

\section{8 \\ MM FISH Panel Validation Findings from Purified Plasma Cells (PPC)}

D.P. Singh-Kahlon, J. Blackson, S. Ahmad, M. Quiam

Cancer Genetics Lab (CGI), Rutherford, N.J., USA

The clinical utility of methods to purify/enrich plasma cells is primarily being applied to newly diagnosed or relapsed cases of multiple myeloma (MM) in genetic labs. Additionally, this method is in use for research studies for MGUS or amyloidosis, wherein abnormal plasma cell clones are usually smaller compared to MM cases.

There are 2 methods developed for clinical use. One is the magnetic separation of the plasma cells by CD138 offered by 2 commercial companies (Miltenyi \& Stem Cell Technologies). The other method is the cytoplasmic immunoglobulin precipitation of the plasma cells, called cIg FISH.
At CGI, we performed FISH validation studies on purified plasma cells (PPC) using the magnetic separation method with the Miltenyi AutoMACS Pro Separator machine. By our proto$\mathrm{col}$, the whole procedure is automatic lasting about 2 hours including a harvest to produce a fixed pellet. A plasma cell FISH validation study was conducted on $25 \mathrm{MM}$ patient samples. Parallel cultures from the same patients were also set up by standard FISH technique. FISH hybridizations were done on a comprehensive FISH panel consisting of rearrangement probes (FGFR3/ $I G H$, CCND1/IGH, MAF/IGH), deletion probes (13q, 17p) and for checking hyperdiploidy (5q, CEP 9). 50 to 100 interphase cells were scored by the purified plasma cell method as compared to 200-300 cells for standard FISH. Two readers scored the cells and interpretation of the results is based on laboratory-established cut-off values.

Plasma cell FISH validation results will be presented in comparison to the standard FISH results. The technique shows the overall advantages of the purified plasma cells method, most importantly in its higher sensitivity. The methodology has also proven advantageous in reducing the tech time during analysis. The higher sensitivity of plasma cell FISH is especially informative to the hematologists making therapy decisions for MM patients.

\section{9}

Multidisciplinary Approach in the Diagnosis of T-PLL P. Starshak ${ }^{a}$, Y. Linhares ${ }^{b}$, P.N. Rao a , C.A. Tirado ${ }^{a}$

Departments of a Pathology and ${ }^{\mathrm{b}}$ Internal Medicine, David Geffen UCLA School of Medicine, Los Angeles, Calif., USA

T-cell prolymphocytic leukemia (T-PLL) is an aggressive Tcell disorder associated with a dismal prognosis. Given the immunophenotypic and morphologic heterogeneity of this leukemia an accurate diagnosis can be difficult and requires a multidisciplinary approach. Herein, we present 2 distinct and diagnostic challenging cases of T-PLL that demonstrate this immunophenotypic and morphologic heterogeneity but showed characteristic cytogenetic abnormalities.

Case 1. 52-year-old-female with WBC count of $189 \times 10^{3} / \mu \mathrm{l}$ (71\% immature lymphocytes). CT-scan showed a mediastinal mass encasing the right main stem bronchus. She was initially incorrectly diagnosed as T-ALL at an outside institution and was started on a treatment regimen of hyper-CVAD. Secondary review of the peripheral blood showed circulating lymphocytes most consistent with prolymphocytes. Flow cytometry showed CD4+, CD52+ T-cells and the bone marrow had similar appearing lymphocytes and immunostains showed strong TCL1 positivity. CDla and TdT were both negative. Cytogenetics showed a complex karyotype with isochromosome $8 \mathrm{q}$, unbalanced rearrangements at $2 \mathrm{p}, 4 \mathrm{p}, 14 \mathrm{q}$ and $18 \mathrm{q}$, monosomy 17 , along with marker chromosomes. Concurrent FISH analysis confirmed $14 \mathrm{q} 11.2$ (TCR alpha/delta) rearrangement and trisomy $8 \mathrm{q}$.

Case 2. 84-year-old asymptomatic male presenting with mildly elevated WBC count of $22.3 \times 10^{3} / \mu$ l (17\% neutrophils, $76 \%$ mature lymphocytes). Lymphocytes were intermediate in size 
with irregular nuclear contours, indistinct nucleoli and cytoplasmic blebs. Flow cytometry showed CD8+, CD52+ T-cells and the bone marrow showed similar appearing atypical lymphocytes that by immunostains were CD8+ with subset of these showing very weak TCL1 expression. A staging CT-scan showed only mild chest lymphadenopathy and no hepatosplenomegaly. Chromosome analysis showed a $\mathrm{t}(\mathrm{X} ; 14)(\mathrm{q} 28 ; \mathrm{q} 11.2)$, deletion $13 \mathrm{q}$, and 22q. FISH detected rearrangement of the TCR alpha/delta gene at $14 \mathrm{q} 11.2$ and trisomy $8 \mathrm{q}$.

These 2 cases of T-PLL highlight the heterogeneity and diagnostic challenge of this disease and demonstrate the importance of integrating morphology, flow cytometry and cytogenetic findings to achieve an accurate diagnosis.

\section{0 \\ An Unbalanced Rearrangement Involving a Translocation $(6 ; 8)$ in a Case of Diffuse Large B-Cell Lymphoma}

C.A. Tirado, J.W. Boles, P. Bui, F. Quintero-Rivera

Clinical Molecular Cytogenetics Laboratory, Department of Pathology \& Laboratory Medicine, David Geffen UCLA School of Medicine, Los Angeles, Calif., USA

Diffuse large B-cell lymphoma (DLBCL) is the most common type of non-Hodgkin lymphoma comprising more than $30 \%$ of adult non-Hodgkin lymphomas in the West. DLBCL represents a diverse set of lymphomas with a diffuse proliferation of large $B$ lymphoid cells. Herein we present a 74-year-old male with lymphadenopathy. Peripheral blood showed leukocytosis with relative lymphocytosis, mild normocytic anemia and mild thrombocytopenia. Flow cytometry studies revealed a CD5-/CD10-/CD103 kappa restricted monoclonal B-cell population composed of 2 small sub-populations of clonal B-cells suggesting the possibility of diffuse large B-cell lymphoma which in many instances can show a heterogeneous morphologic pattern of involvement. Cytogenetic studies of the peripheral blood showed a complex karyotype with numerical abnormalities such as monosomies of chromosomes 2, 4 and 16; trisomies 18 and 19, deletion 9q and $13 \mathrm{q}($ interstitial) and an unbalanced rearrangement leading to a $\mathrm{t}(6 ; 8)$ involving $6 \mathrm{q} 11.2$ and $8 \mathrm{q} 11.2$. To the best of our knowledge, this unbalanced translocation as well as the breakpoints mentioned above are rare events in DLBCL and have been reported in only few cases of diffuse large B-cell lymphoma.

\section{1 \\ Co-Localization and Amplification of $M Y C$ and MLL Oncogenes May Suggest a 'Double Hit' Phenomenon in Acute Myeloid Leukemia (AML)}

Z.-X. Wang, A. Dulau, J. Gong, S. Peiper, R. Bajaj

Cytogenetics Lab, Department of Pathology, Anatomy and Cell Biology, Thomas Jefferson University Hospital, Philadelphia, Pa., USA

Amplification is a classic mechanism for oncogene activation that plays a key role in the biology of multiple malignancies. Oncogene amplification is typically associated with a poor prognosis in human malignancies which include amplification of $M Y C$ and $M L L$ genes. These oncogenes have been shown to be amplified individually in myelodysplasia and acute leukemias. While simultaneous co-amplification of $M Y C$ and $M L L$ genes has been only observed earlier in cell lines, co-localization and co-amplification have not been described.

Here we describe 2 AML cases with a cytogenetic hallmark of gene amplification and increased copies of $M L L$ gene detected by FISH that had involvement of the MYC gene demonstrated using molecular cytogenomic techniques. The first AML case showed a complex karyotype with a ring chromosome and dmin. Fluorescence in situ hybridization (FISH) showed a deletion of p53 at $17 \mathrm{p} 13$ and amplification of the MLL gene. OaCGH confirmed the $M L L$ amplification and $p 53$ loss of heterozygosity detected by FISH and also showed amplification of the MYC gene. Additional FISH performed using both MYC and $M L L$ probes together in one preparation showed a co-localization of both genes in a ring and dmin suggesting a 'double hit' phenomenon with co-localization of 2 oncogenes resulting from translocation of $M Y C$ to the derivative chromosome 11 prior to amplification.

A second AML patient also showed a complex karyotype with marker chromosomes. Simultaneous FISH performed for MYC and $M L L$ genes demonstrated their co-localization and the amplification of the $M L L$ gene.

This phenomenon of co-localization of 2 oncogenes and amplification may raise a question of a 'double hit' phenomenon in AML which could be easily missed if FISH for both MYC and MLL is not performed simultaneously. In conclusion, it is important to identify the mechanism and prognostic impact of the formation of the MYC-MLL co-localization (double-hit) through extended study.

\section{2 \\ Optimized Clinical Stratification of Renal Tumors: Validation of SNP Microarray as a First-Tier Genetic Test for Renal Cell Carcinoma}

I. Znoyko, M.T. Smith, L.N. Scurry, D.J. Wolff

Department of Pathology and Laboratory Medicine, Medical University of South Carolina, Charleston, S.C., USA

Analysis of recurrent genetic lesions characteristic for renal cell carcinoma (RCC) subtypes assists in accurate classification of RCC, particularly in tumors with complex or unusual mor-

Preprint Cytogenet Genome Res 136/4/2012 
phology. However, due to issues related to cell culturing, accuracy of traditional cytogenetic analyses may be compromised. Microarray analysis offers a high resolution alternative tool that may provide improved diagnostic yield. To validate the clinical utility of whole genome SNP microarray for the study of RCC, conventional cytogenetics and microarray SNP analysis were tested for their ability to stratify renal tumors into main RCC subtypes.

Twenty-seven samples of fresh (16/27) and formalin fixed paraffin embedded (11/27) renal tumors were analyzed using both the Illumina Infinium II BeadChip Platform and conventional karyotyping. Results were correlated with gold standard histopathology. Microarray results correlated with morphologic diagnosis in $100 \%(27 / 27)$ of cases, while cytogenetics identified recurrent, subtype defining aberrations in $80 \%(21 / 27)$ of cases. In 2 cases karyotyping produced abnormal but unclassifiable result since subtype defining genetic aberrations were not detected, and in 4 cases, false negative results were obtained by routine chromosome analysis.

Our studies revealed that SNP microarray is the optimal genetic analysis method for clinical diagnosis of renal tumors due to the following reasons: (i) classification of renal tumors into subtypes on the basis of SNP microarray results showed higher concordance with morphologic diagnosis than that of cytogenetics; (ii) the assay can be used on both fresh and archived tissue; (iii) it does not require cell culturing, which speeds up the process and eliminates cell culture associated artifacts and false negative results from stromal cell overgrowth; (iv) the inability of microarrays to detect balanced rearrangements does not limit its capacity to classify renal tumors, since the defining genetic aberrations in RCC are associated with copy number changes and are efficiently determined by microarray. 


\section{Exhibitors/Sponsors}

Thanks to all the exhibitors for supporting this meeting.

Please visit their booths during the breaks.

\begin{abstract}
Abbott Molecular
1300 E. Touhy Ave

Des Plaines, IL 60018

Phone: (800) 553-7042

Fax: (224) 361-7576

Contact: Mike Cerney

Michael.Cerney@abbott.com
\end{abstract}

Affymetrix, Inc.

3420 Central Expressway

Santa Clara, CA 95051

Phone: (408) 731-5047

Fax: (408) 731-5380

Contact: Dara Wright

Dara_Wright@affymetrix.com

\section{Agilent Technologies}

5301 Stevens Creek Blvd

Santa Clara, CA 95051

Phone: (800) 227-9770

Contact: Melissa Leone

Melissa_Leone@agilent.com

Applied Spectral Imaging, Inc.

5315 Avenida Encinas, Suite 150

Carlsbad, CA 92008

Phone: (760) 929-2840

Fax: (760) 929-2842

Contact: Alecia Pritchett

alecia@spectral-imaging.com

BCM Medical Genetics Laboratories

Baylor College of Medicine

One Baylor Plaza, NAB 2015

Houston, TX 77030

Phone: (713) 798-5767

Fax: (713) 798-4187

Contact: Brandon Perthuis

Perthuis@bcm.edu
BioDiscovery, Inc.

2301 Rosecrans Ave., Suite 2185

El Segundo, CA 90245

Phone: (310) 414-8100

Fax: (310) 414-8111

info@biodiscovery.com

BlueGnome Ltd

823 Valencia Avenue

Half Moon Bay, CA 94019

Phone: (650) 284-9732

Fax: (814) 284-8650

Contact: Tony Lialin

tony.lialin@cambridgebluegnome.com

\section{Cartagenia}

Technologielaan 3

BE-3001 Leuven, Belgium

Phone: 0032-16-40-4066

Fax: 0032-16-40-2066

Contact: Herman Verrelst

herman.verrelst@cartagenia.com

CymoGen Dx, LLC

4 Crotty Lane, Suite 100

New Windsor, NY 12553

Phone: (877) 550-4020

Fax: (845) 220-4023

Contact: Peter Hartmayer

Peter.Hartmayer@cymogendx.com

Cytocell/Rainbow Scientific, Inc.

83 Maple Avenue

Windsor, CT 06095

Phone: (860) 298-8382

Fax: (860) 298-8586

Contact: Peter Mousseau

info@rainbowscientific.com
Genial Genetics/

Rainbow Scientific, Inc.

83 Maple Avenue

Windsor, CT 06095

Phone: (860) 298-8382

Fax: (860) 298-8586

Contact: Peter Mousseau

info@rainbowscientific.com

Illumina, Inc.

9885 Towne Center Dr

San Diego, CA 92121

Phone: (203) 823-8507

Fax: (830) 755-5227

Contact: Aysel Heckel

aheckel@illumina.com

\section{InfoQuant}

86 Naxos Building

London E14 8LE, UK

Phone: (617) 475-5167

Fax: (801) 760-5811

Contact: Anton Petrov

info@infoquant.com

Irvine Scientific

2511 Daimler St

Santa Ana, CA 92705

Phone: (949) 261-7800

Fax: (949) 261-6522

Contact: Alan McGrath

amcgrath@irvinesci.com

\section{ISCA Consortium}

2165 N. Decatur Rd

Decatur, GA 30033

Phone: (404) 778-8485

Fax: (404) 778-8562

Contact: Erin Riggs

Erin.Riggs@emory.edu

Preprint Cytogenet Genome Res 136/4/2012 
Kreatech, Inc.

1821 Hillandale Road, Suite 1B-388

Durham, NC 27705

Phone: (919) 405-8347

Contact: Ginny Meihaus

g.meihaus@kreatech.com

\section{Laboratory Corporation of America}

Center for Molecular Biol. \& Path.

1904 Alexander Drive

Research Triangle Park, NC 27709

Phone: (800) 533-0567

Fax: (919) 361-7755

Contact: Jim Tepperberg

Tepperj@LabCorp.com

\section{Leica Microsystems Corp}

120 Baytech Dr.

San Jose, CA 95134

Phone: (408) 719-6407

Fax: (408) 719-6401

Contact: Chris Cruze

Chris.Cruze@leica-microsystems.com

\section{MetaSystems Group, Inc.}

300 Bear Hill Rd

Waltham, MA 02451

Phone: (617) 924-9950

Fax: (617) 924-9954

Contact: Ulrich Klingbeil Bill Hanifin

info@metasystems.org

Nikon Instruments, Inc. 1300 Walt Whitman Rd

Melville, NY 11747

Phone: (800) 52-NIKON

Fax: (631) 547-8652

microscopysales@nikon.net
Olympus America

3500 Corporate Pkwy

Center Valley, PA 18034

Phone: (484) 896-5844

Contact: Lance Hall

Lance.Hall@olympus.com

\section{Oxford Gene Technology}

Begbroke Science Park

Sandy Lane, Yarnton

Oxford OX5 1PF, UK

Phone: +44 (0)1865 856826

Fax: +44 (0)1865 848684

Contact: Spencer Howell

spencer.howell@ogt.co.uk

Phalanx Biotech Group, Inc.

1301 Shoreway Road, Suite 160

Belmont, CA 94002

Phone: (650) 320-8669

Fax: (650) 508-9889

info@OneArray.com

Quest Diagnostics Nichols Institute

14225 Newbrook Drive

Chantilly, VA 20153

Phone: (703) 802-7024

Fax: (703) 802-7103

Contact: Joey Kelly

Joey.C.Kelly@QuestDiagnostics.com

\section{SCC Soft Computer}

5400 Tech Data Drive

Clearwater, FL 33760

Phone: (727) 789-0100

Fax: (727) 789-0124

Contact: Ellie Vahman

ellie@softcomputer.co

\section{SciGene}

470 Lakeside Dr, Ste F

Sunnyvale, CA 94085

Phone: (800) 342-2119

Fax: (408) 733-7336

Contact: Paul Scott

sales@scigene.com

\section{Signature Genomics}

from PerkinElmer, Inc.

2820 North Astor Street

Spokane, WA 99207

Phone: (877) 744-2447

Fax: (509) 474-6839

Info@signaturegenomics.com

South Texas Reference Laboratories

Department of Pathology

University of Texas Health Science Ctr

7703 Floyd Curl Drive

San Antonio, TX 77229

Phone: (210) 567-4050

Fax: (210) 567-4041

Contact: Gopalrao Velagaleti

Velagaleti@uthscsa.edu

\section{Staff Icons}

1 Kalisa Way, Suite 309

Paramus, NJ 07652

Phone: (201) 986-7888

Fax: (201) 986-7444

Contact: Dawn Foster

dfoster@stafficons.com

Transgenomic, Inc.

12325 Emmet St

Omaha, NE 68164

Phone: (401) 619-1691

Fax: (401) 619-1692

Contact: John McCloskey

jmccloskey@transgenomic.com 